Canad. J. Math. Vol. 71 (5), 2019 pp. 1163-1207

http://dx.doi.org/10.4153/CJM-2018-016-2

(c) Canadian Mathematical Society 2019 This is an Open Access article, distributed under

the terms of the Creative Commons Attribution licence (http://creativecommons.org/licenses/by/4.0/), which permits unrestricted re-use, distribution, and reproduction in any medium, provided the original work is properly cited.

\title{
Local Shtukas and Divisible Local Anderson Modules
}

\author{
Urs Hartl and Rajneesh Kumar Singh
}

\begin{abstract}
We develop the analog of crystalline Dieudonné theory for $p$-divisible groups in the arithmetic of function fields. In our theory $p$-divisible groups are replaced by divisible local Anderson modules, and Dieudonné modules are replaced by local shtukas. We show that the categories of divisible local Anderson modules and of effective local shtukas are anti-equivalent over arbitrary base schemes. We also clarify their relation with formal Lie groups and with global objects like Drinfeld modules, Anderson's abelian $t$-modules and $t$-motives, and Drinfeld shtukas. Moreover, we discuss the existence of a Verschiebung map and apply it to deformations of local shtukas and divisible local Anderson modules. As a tool we use Faltings's and Abrashkin's theories of strict modules, which we review briefly.
\end{abstract}

\section{Introduction}

In the arithmetic of number fields, elliptic curves and abelian varieties are important objects. Their theory has been extensively developed in the last two centuries and their moduli spaces have played a major role in Faltings's proof of the Mordell conjecture [Fal83, CS86], the proof of Fermat's Last Theorem by Wiles and Taylor [Wil95, TW95, CSS97], and the proof of the Langlands correspondence for $\mathrm{GL}_{n}$ over nonarchimedean local fields of characteristic zero by Harris and Taylor [HT01]. A useful tool for studying abelian varieties and their moduli spaces are $p$-divisible groups. More precisely, for an elliptic curve or an abelian variety $E$ over a $\mathbb{Z}_{p}$-algebra $R$ the $p$-divisible group $E\left[p^{\infty}\right]=\lim _{\longrightarrow} E\left[p^{n}\right]$, also called the Barsotti-Tate group, captures the

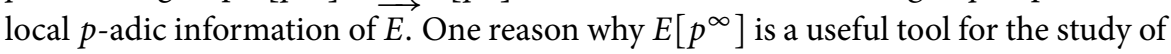
$E$ is that the complicated arithmetic data of a $p$-divisible group over a $\mathbb{Z}_{p}$-algebra $R$ in which $p$ is nilpotent can be faithfully encoded by an object of semi-linear algebra, its Dieudonné module.

Elliptic curves and abelian varieties have analogs in the arithmetic of function fields. Namely, Drinfeld [Dri74, Dri87] invented the notions of elliptic modules (today called Drinfeld modules) and the dual notion of $F$-sheaves (today called Drinfeld shtukas). These structures are function field analogs of elliptic curves in the following sense. Their endomorphism rings are rings of integers in global function fields of positive characteristic or orders in central division algebras over the later. On the other hand, their moduli spaces are varieties over smooth curves over a finite field.

Received by the editors September 29, 2017; revised April 6, 2018.

Published electronically.

Both authors were supported by the Deutsche Forschungsgemeinschaft (DFG) in form of the research grant HA3002/2-1 and the SFB's 478 and 878.

AMS subject classification: 11G09, 13A35, 14L05.

Keywords: local shtuka, formal Drinfeld module, formal t-module. 
Through these two aspects in which global function fields of positive characteristic come into play, Drinfeld shtukas and variants of them proved to be fruitful for establishing large parts of the Langlands program over local and global function fields of positive characteristic in works by Drinfeld [Dri74,Dri77,Dri87], Laumon, Rapoport, and Stuhler [LRS93], L. Lafforgue [Laf02], and V. Lafforgue [Laf18]. Beyond this the analogy between Drinfeld modules and elliptic curves is abundant.

In this spirit, Anderson [And86] introduced higher dimensional generalizations of Drinfeld modules, called abelian t-modules. These are group schemes which carry an action of the polynomial ring $\mathbb{F}_{r}[t]$ over a finite field $\mathbb{F}_{r}$ with $r$ elements subject to certain conditions. Abelian $t$-modules are the function field analogs of abelian varieties [BH09]. Although Anderson worked over a field, abelian $t$-modules also exist naturally over arbitrary $\mathbb{F}_{r}[t]$-algebras $R$ as base rings (Definition 6.5). They possess an (anti-)equivalent description by semi-linear algebra objects called $t$-motives, which are $R[t]$-modules together with a Frobenius semi-linear endomorphism (Definition 6.2 and Theorem 6.6) and are a variant and generalization of Drinfeld shtukas. Through the work of Drinfeld and Anderson it was realized very early on that a Drinfeld module or abelian $t$-module over a field is completely described by its $t$-motive. The same is true over an arbitrary $\mathbb{F}_{r}[t]$-algebra $R$, as is shown for example in [Harl7]. So in a way the situation in function field arithmetic is much better than in the arithmetic of abelian varieties; the $t$-motive is a "global" Dieudonné module that integrates the "local" Dieudonné modules for every prime in a single object.

Correspondingly it is not difficult to come up with a definition of a Dieudonné module at a prime $\mathfrak{p} \subset \mathbb{F}_{r}[t]$ of an abelian $t$-module. It should arise as the $\mathfrak{p}$-adic completion of its $t$-motive; see Example 6.7 (ii) for details. The object one ends up with is an effective local shtuka. To define these, let $\mathfrak{p}=(z)$ for a monic irreducible polynomial $z \in \mathbb{F}_{r}[t]$ and let $\mathbb{F}_{q}=\mathbb{F}_{r}[t] / \mathfrak{p}$ be the residue field. Then $\lim \mathbb{F}_{r}[t] / \mathfrak{p}^{n}=$ $\mathbb{F}_{q}\left[[z]\right.$. Let $R$ be an $\mathbb{F}_{q}[[z]]$-algebra in which the image $\zeta$ of $z$ is nilpotent. An effective local shtuka over $R$ is a pair $\underline{M}=\left(M, F_{M}\right)$ consisting of a locally free $R[\llbracket z]$-module $M$ of finite rank and an isomorphism $F_{M}: \sigma_{q}^{*} M\left[\frac{1}{z-\zeta}\right] \stackrel{\sim}{\rightarrow} M\left[\frac{1}{z-\zeta}\right]$ with $F_{M}\left(\sigma_{q}^{*} M\right) \subset M$. Here $\sigma_{q}^{*}$ is the endomorphism of $R[[z]$ that extends the $q$-Frobenius endomorphism $\sigma_{q}^{*}:=\operatorname{Frob}_{q, R}: b \mapsto b^{q}$ for $b \in R$ by $\sigma_{q}^{*}(z)=z$, and $\sigma_{q}^{*} M:=M \otimes_{R \llbracket z \rrbracket, \sigma_{q}^{*}} R[[z]]$. Now the goal of crystalline Dieudonné theory in the arithmetic of function fields is to describe the analogs of $p$-divisible groups that correspond to effective local shtukas. In the present article we call them $z$-divisible local Anderson modules as in the following definition, and we develop this theory under the technical assumption that $\zeta \in R$ is nilpotent. This theory was already announced in [Har05, Har09, Har11,HK19] and was used in [Har17].

Definition 7.1 A $z$-divisible local Anderson module over $R$ is a sheaf of $\left.\mathbb{F}_{q}[z]\right]$ modules $G$ on the big fppf-site of $\operatorname{Spec} R$ such that

(i) $G$ is $z$-torsion, that is, $G=\lim G\left[z^{n}\right]$, where $G\left[z^{n}\right]:=\operatorname{ker}\left(z^{n}: G \rightarrow G\right)$.

(ii) $G$ is $z$-divisible, that is, $z: G \rightarrow G$ is an epimorphism.

(iii) For every $n$ the $\mathbb{F}_{q}$-module $G\left[z^{n}\right]$ is representable by a finite locally free strict $\mathbb{F}_{q}$-module scheme over $R$ in the sense of Faltings (Definition 4.7). 
(iv) Locally on $\operatorname{Spec} R$ there exists an integer $d \in \mathbb{Z}_{\geq 0}$, such that $(z-\zeta)^{d}=0$ on $\omega_{G}$ where $\omega_{G}:=\lim \omega_{G\left[z^{n}\right]}$ and $\omega_{G\left[z^{n}\right]}:=\varepsilon^{*} \Omega_{G\left[z^{n}\right] / \operatorname{Spec} R}^{1}$ for the unit section $\varepsilon$ of $G\left[z^{n}\right]$ over $R$.

Such objects were studied in the special case with $d=1$ in work by Drinfeld [?], Genestier [Gen96], Laumon [Lau96], Taguchi [Tag93], and Rosen [Ros03]. Generalizations for $d>1$ and their semi-linear algebra description by the analog of Dieudonné theory were attempted by the first author [Har05, Definition 6.2] and by W. Kim [Kim09, Definition 7.3.1]. But unfortunately both definitions and the statements about the analog of Dieudonné theory [Har05, Theorem 7.2] and [Kim09, Theorem 7.3.2] are wrong. The problem lies in the fact that the strictness assumption from (iii) is missing. Our above definition corrects this error. It generalizes Anderson’s [And93, \$3.4] definition of formal $t$-modules that considered the case where the $G\left[z^{n}\right]$ are radicial and $G$ is a formal $\mathbb{F}_{q}[[z]]$-module in the following sense.

Definition 1.1 In this article we define a formal $\mathbb{F}_{q}\left[[z]\right.$-module over an $\mathbb{F}_{q}$-scheme $S$ to be a formal Lie group $G$ equipped with an action of $\mathbb{F}_{q}[[z]$. In particular, we do not impose a condition for the $\mathbb{F}_{q}[[z]]$-action on $\omega_{G}$.

The description of $z$-divisible local Anderson modules by effective local shtukas is deduced from Abrashkin's [Abr06] anti-equivalence between finite locally free strict $\mathbb{F}_{q}$-module schemes over Spec $R$ and finite $\mathbb{F}_{q}$-shtukas. The latter are pairs $\left(M, F_{M}\right)$ consisting of a locally free $R$-module $M$ of finite rank and an $R$-module homomorphism $F_{M}: \sigma_{q}^{*} M \rightarrow M$. We define finite and local shtukas in Section 2 and we recall Abrashkin's results in Section 5. His equivalence is given by Drinfeld's functor

$$
\left(M, F_{M}\right) \mapsto \operatorname{Dr}_{q}\left(M, F_{M}\right):=\operatorname{Spec}\left(\bigoplus_{n \geq 0} \operatorname{Sym}_{R}^{n} M\right) /\left(m^{\otimes q}-F_{M}\left(\sigma_{q}^{*} m\right): m \in M\right),
$$

and its quasi-inverse defined on a finite locally free strict $\mathbb{F}_{q}$-module scheme $G$ as

$$
G \mapsto \underline{M}_{q}(G):=\operatorname{Hom}_{R \text {-groups }, \mathbb{F}_{q}-\operatorname{lin}}\left(G, \mathbb{G}_{a, R}\right),
$$

by which we mean the $R$-module of $\mathbb{F}_{q}$-equivariant morphisms of group schemes over $R$ on which the Frobenius $F_{M_{q}(G)}$ is provided by the relative $q$-Frobenius of the additive group scheme $\mathbb{G}_{a, R}$ over $R$. Various properties of $\underline{M}$ are reflected in properties of $\operatorname{Dr}_{q}(\underline{M})$ (Theorem 5.2). The functors $\operatorname{Dr}_{q}$ and $\underline{M}_{q}$ are extended to effective local shtukas $\underline{M}$ and $z$-divisible local Anderson modules $G$ by

$$
\underline{M} \mapsto \operatorname{Dr}_{q}(\underline{M}):=\underset{n}{\lim _{n}} \operatorname{Dr}_{q}\left(\underline{M} / z^{n} \underline{M}\right), \quad G \mapsto \underline{M}_{q}(G):={\underset{\lim }{\leftarrow}}_{n} \underline{M}_{q}\left(G\left[z^{n}\right]\right) .
$$

Generalizing Anderson [And93, \$3.4], who treated the case of formal $\mathbb{F}_{q}[[z]]$-modules, we prove the following theorem.

Theorem $8.3 \quad$ (i) The two contravariant functors $\operatorname{Dr}_{q}$ and $\underline{M}_{q}$ are mutually quasiinverse anti-equivalences between the category of effective local shtukas over $R$ and the category of z-divisible local Anderson modules over $R$. 
(ii) Both functors are $\mathbb{F}_{q}[[z]]$-linear, map short exact sequences to short exact sequences, and preserve (ind-)étale objects.

(iii) $G$ is a formal $\mathbb{F}_{q}[z] \rrbracket$-module if and only if $F_{M}$ is topologically nilpotent, that is $\operatorname{im}\left(F_{M}^{n}\right) \subset z M$ for an integer $n$.

(iv) The $R\left[[z]\right.$-modules $\omega_{\operatorname{Dr}_{q}\left(M, F_{M}\right)}$ and coker $F_{M}$ are canonically isomorphic.

In Section 6 we explain the relation of $z$-divisible local Anderson modules and local shtukas to global objects like Drinfeld modules [Dri74], Anderson's [And86] abelian $t$-modules and $t$-motives, and Drinfeld shtukas [Dri87]. In particular, if $E$ is a Drinfeld- $\mathbb{F}_{r}[t]$-module or an abelian $t$-module over $R$, then the $z^{n}$-torsion points $E\left[z^{n}\right]$ of $E$ form a finite locally free $\mathbb{F}_{r}[t] /\left(z^{n}\right)$-module scheme over $R$. By Example 6.7 (ii), the limit $G:=E\left[z^{\infty}\right]:=\lim E\left[z^{n}\right]$ in the category of fppf-sheaves of $\mathbb{F}_{q}[[z]]$-modules on $\operatorname{Spec} R$ satisfies $G\left[\overrightarrow{z^{n}}\right]:=\operatorname{ker}\left(z^{n}: G \rightarrow G\right)=E\left[z^{n}\right]$ and is a $z$-divisible local Anderson module over $R$. Moreover, the associated effective local shtuka $\underline{M}_{q}(G)$ from Theorem 8.3 arises as the $z$-adic completion of the $t$-motive associated with $E$; see Example 6.7 (ii).

In Section 7 we present the above definition of $z$-divisible local Anderson modules $G$ and give equivalent definitions. We also introduce truncated $z$-divisible local Anderson modules such as $G\left[z^{n}\right]$ (Proposition 9.5). In Section 9 we investigate, for $\zeta=0$ in $R$, the existence of a $z^{d}$-Verschiebung $V_{z^{d}, G}$ for (truncated) $z$-divisible local Anderson modules $G$, respectively for local shtukas, with $V_{z^{d}, G} \circ F_{q, G}=z^{d} \cdot \operatorname{id}_{G}$ and $F_{q, G} \circ V_{z^{d}, G}=z^{d} \cdot \operatorname{id}_{\sigma_{q}^{*} G}$, where $F_{q, G}$ is the relative $q$-Frobenius of $G$ over $R$. We use the $z^{d}$-Verschiebung in Theorem 9.8 to prove that lifting a $z$-divisible local Anderson module from $R / I$ to $R$, when $I^{q}=(0)$, is equivalent to lifting the Hodge filtration on its de Rham cohomology. In Section 10 we use the $z^{d}$-Verschiebung to clarify the relation between $z$-divisible local Anderson modules $G$ and formal $\mathbb{F}_{q}[z] \rrbracket$ modules. Following the approach of Messing [Mes72], who treated the analogous situation of $p$-divisible groups and formal Lie groups, we show that a $z$-divisible local Anderson module is formally smooth (Theorem 10.4) and how to associate a formal $\mathbb{F}_{q}[[z]]$-module with it (Theorem 10.7). We also discuss conditions under which it is an extension of an (ind-)étale $z$-divisible local Anderson module by a $z$-divisible formal $\left.\mathbb{F}_{q}[z]\right]$-module (Proposition 10.16) and we prove the following corollary.

Corollary 10.12 There is an equivalence of categories between that of $z$-divisible local Anderson modules over $R$ with $G[z]$ radicial, and the category of $z$-divisible formal $\mathbb{F}_{q}[[z]]$-modules $G$ with $G[z]$ representable by a finite locally free group scheme, such that locally on Spec $R$ there is an integer $d$ with $(z-\zeta)^{d}=0$ on $\omega_{G}$.

In Section 4 we explain Faltings's notion of strict $\mathbb{F}_{q}$-module schemes and give details additional to the treatments of Faltings [Fal02] and Abrashkin [Abr06]. This notion is based on certain deformations of finite locally free group schemes and the associated cotangent complex, which we review in Section 3. There is an equivalent description of finite locally free strict $\mathbb{F}_{q}$-module schemes by Poguntke [Pog17] (Remark 5.3). 
Notation 1.2 Let $\mathbb{F}_{q}$ be a finite field with $q$ elements and characteristic $p$. For a scheme $S$ over $\operatorname{Spec} \mathbb{F}_{q}$ and a positive integer $n \in \mathbb{N}_{>0}$, we denote by

$$
\sigma_{q^{n}}:=\operatorname{Frob}_{q^{n}, S}: S \longrightarrow S
$$

its absolute $q^{n}$-Frobenius endomorphism which acts as the identity on points and as the $q^{n}$-power map $b \mapsto b^{q^{n}}$ on the structure sheaf. For an $S$-scheme $X$, respectively, an $\mathcal{O}_{S}$-module $M$, we write $\sigma_{q^{n}}^{*} X:=X \times_{S, \sigma_{q^{n}}} S$, respectively, $\sigma_{q^{n}}^{*} M:=M \otimes_{\mathcal{O}_{S}, \sigma_{q^{n}}^{*}} \mathcal{O}_{S}$, for the pullback under $\sigma_{q^{n}}$. For $m \in M$, we also write $\sigma_{q^{n}}^{*}(m):=m \otimes 1 \in \sigma_{q^{n}}^{*} M$ and note that $\sigma_{q^{n}}^{*}(b m)=b m \otimes 1=m \otimes b^{q^{n}}=b^{q^{n}} \cdot \sigma_{q}^{*} m$ for $b \in \mathcal{O}_{S}$ and $m \in M$.

Let $z$ be an indeterminant over $\mathbb{F}_{q}$. Let $\mathcal{O}_{S}[[z]]$ be the sheaf on $S$ of formal power series in $z$. That is, $\Gamma\left(U, \mathcal{O}_{S}[[z]]\right)=\Gamma\left(U, \mathcal{O}_{S}\right)[[z]]$ for open $U \subset S$ with the obvious restriction maps. This is indeed a sheaf being the countable direct product of $\mathcal{O}_{S}$. Let $\zeta$ be an indeterminant over $\mathbb{F}_{q}$ and let $\mathbb{F}_{q}[[\zeta]]$ be the ring of formal power series in $\zeta$ over $\mathbb{F}_{q}$. Let $\mathcal{N i l p}_{\mathbb{F}_{q}[\zeta]}$ be the category of $\mathbb{F}_{q}[\zeta \zeta]$-schemes on which $\zeta$ is locally nilpotent. For $S \in \mathcal{N i l p}_{\mathbb{F}_{q}[[\zeta]]}$, let $\mathcal{O}_{S}((z))$ be the sheaf of $\mathcal{O}_{S}$-algebras on $S$ associated with the presheaf $U \mapsto \Gamma\left(U, \mathcal{O}_{U}\right)\left[[z]\left[\frac{1}{z}\right]\right.$. If $U$ is quasi-compact, then $\mathcal{O}_{S}((z))(U)=\Gamma\left(U, \mathcal{O}_{S}[[z]]\right)\left[\frac{1}{z}\right]$. Since $\zeta$ is locally nilpotent on $S$, the sheaf $\mathcal{O}_{S}((z))$ equals the sheaf associated with the presheaf $U \mapsto \Gamma\left(U, \mathcal{O}_{S}[[z]]\right)\left[\frac{1}{z-\zeta}\right]$. We denote by $\sigma_{q}^{*}$ the endomorphism of $\mathcal{O}_{S}[[z]]$ and $\mathcal{O}_{S}((z))$ that acts as the identity on $z$ and as $b \mapsto b^{q}$ on local sections $b \in \mathcal{O}_{S}$. For a sheaf $M$ of $\mathcal{O}_{S}[[z]]$-modules on $S$, we let $\sigma_{q}^{*} M:=M \otimes_{\mathcal{O}_{s}}\left[[z], \sigma_{q}^{*} \mathcal{O}_{S}[[z]]\right.$ and

$$
M\left[\frac{1}{z-\zeta}\right]:=M \underset{\mathcal{O}_{S}[[z]}{\otimes} \mathcal{O}_{S}[[z]]\left[\frac{1}{z-\zeta}\right]=M \underset{\mathcal{O}_{S}[[z]]}{\otimes} \mathcal{O}_{S}((z))
$$

be the tensor product sheaves. Also for a section $m \in M$ we write $\sigma_{q}^{*} m:=m \otimes 1 \epsilon$ $\sigma_{q}^{*} M$. Note that by [HV11, Proposition 2.3], a sheaf $M$ of $\mathcal{O}_{S}[[z]]$-modules which fpqclocally on $S$ is isomorphic to $\mathcal{O}_{S}[[z]]^{\oplus r}$ is already Zariski-locally on $S$ isomorphic to $\mathcal{O}_{S}[[z]]^{\oplus r}$. We therefore call such a sheaf simply a locally free sheaf of $\mathcal{O}_{S}[[z]]$-modules of rank $r$.

\section{Local and Finite Shtukas}

Let $S$ be a scheme in $\mathcal{N i l p}_{\mathbb{F}_{q}[[\zeta]]}$.

Definition 2.1 A local shtuka of rank (or height) $r$ over $S$ is a pair $\underline{M}=\left(M, F_{M}\right)$ consisting of a locally free sheaf $M$ of $\mathcal{O}_{S}[[z]]$-modules of rank $r$, and an isomorphism $F_{M}: \sigma_{q}^{*} M\left[\frac{1}{z-\zeta}\right] \stackrel{\sim}{\rightarrow} M\left[\frac{1}{z-\zeta}\right]$

A morphism of local shtukas $f:\left(M, F_{M}\right) \rightarrow\left(M^{\prime}, F_{M^{\prime}}\right)$ over $S$ is a morphism of the underlying sheaves $f: M \rightarrow M^{\prime}$ that satisfies $F_{M^{\prime}} \circ \sigma_{q}^{*} f=f \circ F_{M}$.

A quasi-isogeny between local shtukas $f:\left(M, F_{M}\right) \rightarrow\left(M^{\prime}, F_{M^{\prime}}\right)$ over $S$ is an isomorphism of $\mathcal{O}_{S}((z))$-modules $f: M \otimes_{\left.\mathcal{O}_{S} \llbracket z\right]} \mathcal{O}_{S}((z)) \stackrel{\sim}{\rightarrow} M^{\prime} \otimes_{\left.\mathcal{O}_{s} \llbracket z\right] \rrbracket} \mathcal{O}_{S}((z))$ with $F_{M^{\prime}} \circ$ $\sigma_{q}^{*}(f)=f \circ F_{M}$. A morphism that is a quasi-isogeny is called an isogeny. 
For any local shtuka $\left(M, F_{M}\right)$ over $S \in \mathcal{N} \operatorname{Nilp}_{\left.\mathbb{F}_{q} \llbracket \zeta\right]}$ the homomorphism

$$
M \longrightarrow M\left[\frac{1}{z-\zeta}\right]
$$

is injective by the flatness of $M$ and the following lemma.

Lemma 2.2 Let $R$ be an $\mathbb{F}_{q}[[\zeta]]$-algebra in which $\zeta$ is nilpotent. Then the sequence of $R[[z]]$-modules

$$
0 \longrightarrow R[[z]] \underset{1 \mapsto z-\zeta}{\longrightarrow} R[[z]] \underset{z \mapsto \zeta}{\longrightarrow} R \longrightarrow 0
$$

is exact. In particular $R[[z]] \subset R[[z]]\left[\frac{1}{z-\zeta}\right]$.

Proof If $\sum_{i} b_{i} z^{i}$ lies in the kernel of the first map, that is, $0=(z-\zeta)\left(\sum_{i} b_{i} z^{i}\right)=$ $\sum_{i}\left(b_{i-1}-\zeta b_{i}\right) z^{i}$, then $b_{i}=\zeta b_{i+1}=\zeta^{n} b_{i+n}$ for all $n$. Since $\zeta$ is nilpotent, all $b_{i}$ are zero. Also due to the nilpotency of $\zeta$, the second map is well defined and surjective. For exactness in the middle note that $\sum_{i} b_{i} \zeta^{i}=0$ implies $\sum_{i} b_{i} z^{i}=\sum_{i} b_{i}\left(z^{i}-\zeta^{i}\right)$, which is a multiple of $z-\zeta$.

For a morphism $f: S^{\prime} \rightarrow S$ in $\mathcal{N i l p}_{\left.\mathbb{F}_{q} \llbracket \zeta\right]}$ we can pull back a local shtuka $\left(M, F_{M}\right)$ over $S$ to the local shtuka $\left(M \otimes_{\left.\left.\mathcal{O}_{S} \llbracket z\right]\right]} \mathcal{O}_{S^{\prime}}\left[[z], F_{M} \otimes\right.\right.$ id $)$ over $S^{\prime}$.

We define the tensor product of two local shtukas $\left(M, F_{M}\right)$ and $\left(N, F_{N}\right)$ over $S$ as the local shtuka $\left(M \otimes_{\mathcal{O}_{S}[[z]]} N, F_{M} \otimes F_{N}\right)$. The local shtuka

$$
\underline{1}(0):=\left(\mathcal{O}_{S}[[z]], F_{1(0)}=\operatorname{id}_{\mathcal{O}_{s} \llbracket[z]}: \sigma_{q}^{*} \mathcal{O}_{S}[[z]]=\mathcal{O}_{S}[[z]] \stackrel{\sim}{\longrightarrow} \mathcal{O}_{S}[[z]]\right)
$$

is a unit object for the tensor product. The dual $\left(M^{\vee}, F_{M^{\vee}}\right)$ of a local shtuka $\left(M, F_{M}\right)$

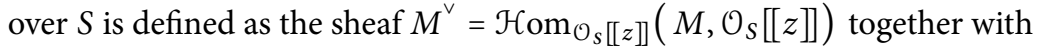

$$
F_{M^{\vee}}: \sigma_{q}^{*} M^{\vee}\left[\frac{1}{z-\zeta}\right] \stackrel{\sim}{\longrightarrow} M^{\vee}\left[\frac{1}{z-\zeta}\right], f \mapsto f \circ F_{M}^{-1}
$$

Also there is a natural definition of internal Hom, given by $\mathcal{H} \operatorname{om}(\underline{M}, \underline{N})=\underline{M}^{\vee} \otimes \underline{N}$. This makes the category of local shtukas over $S$ into an $\mathbb{F}_{q}[[z]$-linear, additive, rigid tensor category. It is an exact category in the sense of Quillen [Qui73, §2], provided one calls a short sequence of local shtukas exact when the underlying sequence of sheaves of $\mathcal{O}_{S}[[z]]$-modules is exact.

Lemma 2.3 Let $\left(M, F_{M}\right)$ be a local shtuka over $S$. Then locally on $S$ there are $e$, $e^{\prime}, N \in \mathbb{Z}$ such that $(z-\zeta)^{e^{\prime}} M \subset F_{M}\left(\sigma_{q}^{*} M\right) \subset(z-\zeta)^{-e} M$ and $z^{N} M \subset F_{M}\left(\sigma_{q}^{*} M\right)$. For any such e the map $F_{M}: \sigma_{q}^{*} M \rightarrow(z-\zeta)^{-e} M$ is injective, and the quotient

$$
(z-\zeta)^{-e} M / F_{M}\left(\sigma_{q}^{*} M\right)
$$

is a locally free $\mathcal{O}_{S}$-module of finite rank.

Proof We work locally on $\operatorname{Spec} R \subset S$ and assume that $\sigma_{q}^{*} M$ and $M$ are free $\mathcal{O}_{S}[[z]]$-modules. Applying $F_{M}$ to a basis of $\sigma_{q}^{*} M$, respectively, $F_{M}^{-1}$ to a basis of $M$, proves the existence of $e$, respectively, $e^{\prime}$. If $N \geq e^{\prime}$ is an integer that is a power of $p$ such that $\zeta^{N}=0$ in $R$, then $z^{N} M=\left(z^{N}-\zeta^{N}\right) M=(z-\zeta)^{N} M \subset F_{M}\left(\sigma_{q}^{*} M\right)$. 
We prove that the quotient $K:=(z-\zeta)^{-e} M / F_{M}\left(\sigma_{q}^{*} M\right)$ is a locally free $R$-module of finite rank. This was already proved in [HV11, Lemma 4.3], but the argument given there only works if $R$ is noetherian, because it uses the fact that $R[[z]]$ is flat over $R$. We now give a proof in the non-noetherian case. Since

$K=\operatorname{coker}\left(F_{M} \bmod (z-\zeta)^{e+e^{\prime}}: \sigma_{q}^{*} M /(z-\zeta)^{e+e^{\prime}} \sigma_{q}^{*} M \longrightarrow(z-\zeta)^{-e} M /(z-\zeta)^{e^{\prime}} M\right)$,

it is of finite presentation over $R$. Since $R[[z]] \subset R[[z]]\left[\frac{1}{z-\zeta}\right]$ is a subring by Lemma 2.2 and $M$ is locally free, the map $F_{M}: \sigma_{q}^{*} M \rightarrow(z-\zeta)^{-e} M$ is injective. Let $\mathfrak{m} \subset R$ be a maximal ideal and set $k=R / \mathfrak{m}$. In the exact sequence

$$
\begin{aligned}
& 0 \longrightarrow \operatorname{Tor}_{1}^{R[z]]}(K, k[[z]]) \longrightarrow \sigma_{q}^{*} M \underset{R[[z]]}{\otimes} k[[z]] \longrightarrow(z-\zeta)^{-e} M \underset{R[[z]}{\otimes} k[[z]] \\
& \longrightarrow K \underset{R[[z]]}{\otimes} k[[z]] \longrightarrow 0,
\end{aligned}
$$

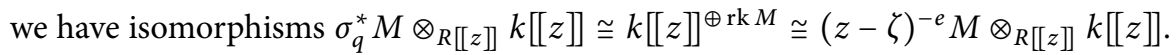
Moreover, $\zeta=0$ in $k$ and hence $z^{e+e^{\prime}} K \otimes_{R[[z]} k[[z]]=0$. Since $k[[z]]$ is a PID, the map $\sigma_{q}^{*} M \otimes_{R[[z]} k[[z]] \rightarrow(z-\zeta)^{-e} M \otimes_{R[[z]]} k[[z]$ is injective by the elementary divisor theorem, and hence $0=\operatorname{Tor}_{1}^{R[[z]]}(K, k[[z]])$. To relate this to

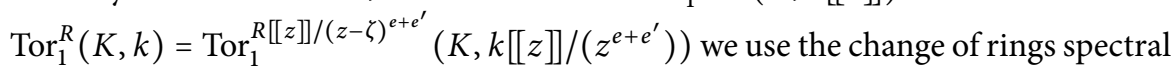
sequence [Rot09, Theorem 10.71] and the induced epimorphism (from its associated 5-term sequence of low degrees, see [Rot09, Theorem 10.31])

$$
\cdots \longrightarrow \operatorname{Tor}_{1}^{R[[z]]}(K, k[[z]]) \longrightarrow \operatorname{Tor}_{1}^{R[[z]] /(z-\zeta)^{e+e^{\prime}}}\left(K, k[[z]] /\left(z^{e+e^{\prime}}\right)\right) \longrightarrow 0 .
$$

It follows that $\operatorname{Tor}_{1}^{R}(K, k)=0$ and from Nakayama's lemma we conclude that $K$ is locally free over $R$ of finite rank; compare [Eis95, Exercise 6.2].

Definition 2.4 A local shtuka $\underline{M}=\left(M, F_{M}\right)$ over $S$ is called effective if $F_{M}$ is actually a morphism $F_{M}: \sigma_{q}^{*} M \hookrightarrow M$. Let $\left(M, F_{M}\right)$ be effective of rank $r=\operatorname{rk} \underline{M}$. We say that

(i) $\left(M, F_{M}\right)$ has dimension $d$ if coker $F_{M}$ is locally free of rank $d$ as an $\mathcal{O}_{S}$-module.

(ii) $\left(M, F_{M}\right)$ is étale if $F_{M}: \sigma_{q}^{*} M \stackrel{\sim}{\rightarrow} M$ is an isomorphism.

(iii) $F_{M}$ is topologically nilpotent if locally on $S$ there is an integer $n$ such that

$$
\begin{gathered}
\operatorname{im}\left(F_{M}^{n}\right) \subset z M, \\
\text { where } F_{M}^{n}:=F_{M} \circ \sigma_{q}^{*} F_{M} \circ \cdots \circ \sigma_{q^{n-1}}^{*} F_{M}: \sigma_{q^{n}}^{*} M \rightarrow M .
\end{gathered}
$$

Example 2.5 We define the Tate objects in the category of local shtukas over $S$ as

$$
\underline{1}(n):=\left(\mathcal{O}_{S}\left[[z], F_{M}: 1 \mapsto(z-\zeta)^{n}\right) .\right.
$$

By Lemma 2.3 every local shtuka over a quasi-compact scheme $S$ becomes effective after tensoring with a suitable Tate object.

More generally, now let $S$ be an arbitrary $\mathbb{F}_{q}$-scheme.

Definition 2.6 A finite $\mathbb{F}_{q}$-shtuka over $S$ is a pair $\underline{M}=\left(M, F_{M}\right)$ consisting of a locally free $\mathcal{O}_{S}$-module $M$ on $S$ of finite rank denoted $\mathrm{rk} \underline{M}$, and an $\mathcal{O}_{S}$-module homomorphism $F_{M}: \sigma_{q}^{*} M \rightarrow M$. A morphism $f:\left(M, F_{M}\right) \rightarrow\left(M^{\prime}, F_{M^{\prime}}\right)$ of finite $\mathbb{F}_{q}$-shtukas 
is an $\mathcal{O}_{S}$-module homomorphism $f: M \rightarrow M^{\prime}$ satisfying $f \circ F_{M}=F_{M^{\prime}} \circ \sigma_{q}^{*} f$. We denote the category of finite $\mathbb{F}_{q}$-shtukas over $S$ by $\mathbb{F}_{q}$-Sht ${ }_{S}$.

A finite $\mathbb{F}_{q}$-shtuka over $S$ is called étale if $F_{M}$ is an isomorphism. We say that $F_{M}$ is nilpotent if there is an integer $n$ such that $F_{M}^{n}:=F_{M} \circ \sigma_{q}^{*} F_{M} \circ \cdots \circ \sigma_{q^{n-1}}^{*} F_{M}=0$.

Finite $\mathbb{F}_{q}$-shtukas were studied at various places in the literature. They were called (finite) $\varphi$-sheaves by Drinfeld [Dri87, \$2], Taguchi and Wan [Tag95, TW96] and Dieudonné $\mathbb{F}_{q}$-modules by Laumon [Lau96]. Finite $\mathbb{F}_{q}$-shtukas over a field admit a canonical decomposition.

Proposition 2.7 ([Lau96, Lemma B.3.10]) If S is the spectrum of a field L, every finite $\mathbb{F}_{q}$-shtuka $\underline{M}=\left(M, F_{M}\right)$ is canonically an extension of finite $\mathbb{F}_{q}$-shtukas

$$
0 \longrightarrow\left(M_{\text {ét }}, F_{\text {ét }}\right) \longrightarrow\left(M, F_{M}\right) \longrightarrow\left(M_{\text {nil }}, F_{\text {nil }}\right) \longrightarrow 0 \text {, }
$$

where $F_{\mathrm{et}}$ is an isomorphism and $F_{\mathrm{nil}}$ is nilpotent, and $\underline{M}_{\mathrm{e}}=\left(M_{\mathrm{e}}, F_{\mathrm{e}}\right)$ is the largest étale finite $\mathbb{F}_{q}$-sub-shtuka of $\underline{M}$ and equals $\operatorname{im}\left(F_{M}^{\mathrm{rk}} \underline{M}\right)$. If $L$ is perfect, this extension splits canonically.

Proof This was proved by Laumon [Lau96, Lemma B.3.10] for perfect $L$. In general one considers the descending sequence $\cdots \supset \operatorname{im}\left(F_{M}^{n}\right) \supset \operatorname{im}\left(F_{M}^{n+1}\right) \supset \cdots$ of $L$ subspaces of $M$ that stabilizes at some finite $n$. If $\operatorname{im}\left(F_{M}^{n+1}\right)=\operatorname{im}\left(F_{M}^{n}\right)$, then

$$
F_{M}: \sigma_{q}^{*}\left(\operatorname{im} F_{M}^{n}\right) \rightarrow \operatorname{im} F_{M}^{n+1}=\operatorname{im} F_{M}^{n}
$$

is surjective, hence bijective, and therefore $\operatorname{im}\left(F_{M}^{n^{\prime}}\right)=\operatorname{im}\left(F_{M}^{n}\right)$ for all $n^{\prime} \geq n$. So the sequence stabilizes already for some $n \leq \mathrm{rk} \underline{M}$ and $M_{\mathrm{ét}}=\operatorname{im}\left(F_{M}^{\mathrm{rk}} \underline{\underline{M}}\right)$. If $L$ is perfect, $M_{\text {nil }}$ is isomorphic to the submodule $\cup_{n \geq 0} \operatorname{ker}\left(F_{M}^{n} \circ \sigma_{q^{n}}^{*}: M \rightarrow M\right)$ of $M$; see [Lau96, Lemma B.3.10].

Example 2.8 Every effective local shtuka $\left(M, F_{M}\right)$ of rank $r$ over $S$ yields for every $n \in \mathbb{N}$ a finite $\mathbb{F}_{q^{-}}$shtuka $\left(M / z^{n} M, F_{M} \bmod z^{n}\right)$ of rank $r n$, and $\left(M, F_{M}\right)$ equals the projective limit of these finite $\mathbb{F}_{q}$-shtukas.

Thus from Proposition 2.7 we obtain the following result.

Proposition 2.9 If $S$ is the spectrum of a field $L$ in $\mathcal{N i l p}_{\mathbb{F}_{q} \llbracket \zeta \rrbracket}$, every effective local shtuka $\left(M, F_{M}\right)$ is canonically an extension of effective local shtukas

$$
0 \longrightarrow\left(M_{\text {ét }}, F_{\text {ét }}\right) \longrightarrow\left(M, F_{M}\right) \longrightarrow\left(M_{\text {nil }}, F_{\text {nil }}\right) \longrightarrow 0,
$$

where $F_{\mathrm{e}}$ is an isomorphism, $F_{\mathrm{nil}}$ is topologically nilpotent, and $\left(M_{\mathrm{e}}, F_{\mathrm{et}}\right)$ is the largest étale effective local sub-shtuka of $\left(M, F_{M}\right)$. If $L$ is perfect, this extension splits canonically.

\section{Review of Deformations of Finite Locally Free Group Schemes}

For a commutative group scheme $G$ over $S$, we denote by $\varepsilon_{G}: S \rightarrow G$ its unit section and by $\omega_{G}:=\varepsilon_{G}^{*} \Omega_{G / S}^{1}$ its co-Lie module. It is a sheaf of $\mathcal{O}_{S}$-modules. In order 
to describe which group objects are classified by finite $\mathbb{F}_{q}$-shtukas, we need to review the definition of a strict $\mathbb{F}_{q}$-module scheme in the next two sections. We follow Faltings [Fal02] and Abrashkin [Abr06]. We begin this section with a review of deformations of finite locally free group schemes. Recall that a group scheme $G$ over $S$ is called finite locally free over $S$ if on every open affine $\operatorname{Spec} R \subset S$ the scheme $G$ is of the form Spec $A$ for a finite locally free $R$-module $A$. By [EGA, $\mathrm{I}_{\text {new }}$, Proposition 6.2.10] this is equivalent to $G$ being finite flat and of finite presentation over $S$. The rank of the $R$-module $A$ is called the order of $G$ and is denoted ord $G$. It is a locally constant function on $S$. The following facts will be used throughout.

Remark 3.1 $\quad$ (a) A morphism $G^{\prime} \rightarrow G$ of finite locally free group schemes is a monomorphism (of schemes, or equivalently of fppf-sheaves on $S$ ) if and only if it is a closed immersion by [EGA, $\mathrm{IV}_{4}$, Corollaire 18.12.6], because it is proper.

(b) Let $G$ and $G^{\prime \prime}$ be group schemes over $S$ that are finite and of finite presentation, and assume that $G$ is flat over $S$. Then a morphism $G \rightarrow G^{\prime \prime}$ is an epimorphism of fppfsheaves on $S$ if and only if it is faithfully flat; compare the proof of [Mes72, Chapter I, Lemma 1.5(b)].

(c) A sequence $0 \rightarrow G^{\prime} \rightarrow G \rightarrow G^{\prime \prime} \rightarrow 0$ of finite locally free group schemes over $S$ is called exact if it is exact when viewed as a sequence of fppf-sheaves on $S$. By the above this is equivalent to the conditions that $G \rightarrow G^{\prime \prime}$ is faithfully flat, and that $G^{\prime} \rightarrow G$ is a closed immersion that equals the kernel of $G \rightarrow G^{\prime \prime}$.

(d) If $G^{\prime} \hookrightarrow G$ is a closed immersion of finite locally free group schemes over $S$, then the quotient $G / G^{\prime}$ exists as a finitely presented group scheme over $S$ by [SGA 3, Théorème V.4.1 and Proposition V.9.1], which is flat by [EGA, IV ${ }_{3}$, Corollaire 11.3.11]. It is integral over $S$ and hence finite, because $\mathcal{O}_{G / G^{\prime}} \subset \mathcal{O}_{G}$. In particular, $G / G^{\prime}$ is finite locally free over $S$.

In the following we will work locally on $S$ and assume that $S=\operatorname{Spec} R$ is affine. Let $G=\operatorname{Spec} A$ be a finite locally free group scheme over $S$. Then $G$ is a relative complete intersection by [SGA 3, Proposition III.4.15]. This means that locally on $S$ we can take $A=R\left[X_{1}, \ldots, X_{n}\right] / I$ where the ideal $I$ is generated by a regular sequence $\left(f_{1}, \ldots, f_{n}\right)$ of length $n, c f$. [EGA, IV ${ }_{4}$, Proposition 19.3.7]. The unit section $\varepsilon_{G}: S \rightarrow G$ defines an augmentation $\varepsilon_{A}:=\varepsilon_{G}^{*}: A \rightarrow R$ of the $R$-algebra $A$, that is, $\varepsilon_{A}$ is a section of the structure morphism $\iota_{A}: R \rightarrow A$. Faltings [Fal02] and Abrashkin [Abr06] defined deformations of augmented $R$-algebras as follows. For every augmented $R$-algebra $\left(A, \varepsilon_{A}: A \rightarrow R\right)$ set $I_{A}:=\operatorname{ker} \varepsilon_{A}$. For the polynomial ring $R[\underline{X}]=R\left[X_{1}, \ldots, X_{n}\right]$ set $I_{R[\underline{X}]}=\left(X_{1}, \ldots, X_{n}\right)$ and $\varepsilon_{R[\underline{X}]}: R[\underline{X}] \rightarrow R, X_{v} \mapsto 0$. Abrashkin [Abr06, \$\$1.1, 1.2] made the following definition.

Definition 3.2 The category DSch $\mathrm{S}_{S}$ has as objects all triples $\mathcal{H}=\left(H, H^{b}, i_{\mathcal{H}}\right)$, where $H=\operatorname{Spec} A$ for an augmented $R$-algebra $A$ that is finite locally free as an $R$-module, where $H^{b}=\operatorname{Spec} A^{b}$ for an augmented $R$-algebra $A^{b}$, and where $i_{\mathcal{H}}: H \leftrightarrow H^{\mathrm{b}}$ is a closed immersion given by an epimorphism $i_{\mathcal{A}}: A^{b} \rightarrow A$ of augmented $R$-algebras, such that locally on $\operatorname{Spec} R$ there is a polynomial ring $R[\underline{X}]=R\left[X_{1}, \ldots, X_{n}\right]$ and an epimorphism of augmented $R$-algebras $j: R[\underline{X}] \rightarrow A^{b}$ satisfying the properties that 
(i) the ideal $I:=\operatorname{ker}\left(i_{\mathcal{A}} \circ j\right)$ is generated by elements of a regular sequence of length $n$ in $R[\underline{X}]$,

(ii) ker $j=I \cdot I_{R[\underline{X}]}$, and hence $A=R[\underline{X}] / I$ and $A^{b}=R[\underline{X}] /\left(I \cdot I_{R[\underline{X}]}\right)$.

In particular, $H$ is a relative complete intersection. We write $\mathcal{A}=\left(A, A^{\mathrm{b}}, i_{\mathcal{A}}\right)$ and $\mathcal{H}=\operatorname{Spec} \mathcal{A}$. A morphism $\operatorname{Spec}\left(\widetilde{A}, \widetilde{A}^{b}, i_{\widetilde{\mathcal{A}}}\right) \rightarrow \operatorname{Spec}\left(A, A^{b}, i_{\mathcal{A}}\right)$ in $\operatorname{DSch}_{S}$ is given by morphisms $f: A \rightarrow \widetilde{A}$ and $f^{b}: A^{b} \rightarrow \widetilde{A}^{b}$ of augmented $R$-algebras such that $f \circ i_{\mathcal{A}}=$ $i_{\widetilde{\mathcal{A}}} \circ f^{b}$. Sometimes $i_{\mathcal{H}}$ and $i_{\mathcal{A}}$ are omitted.

For an object $\mathcal{H}=\operatorname{Spec}\left(A, A^{b}, i_{\mathcal{A}}\right)$ of $\mathrm{DSch} S$, define the two $R$-modules $N_{\mathcal{H}}=$ $\operatorname{ker} i_{\mathcal{A}}$ and $t_{\mathcal{H}}^{*}=I_{A^{b}} / I_{A^{b}}^{2}$, where $I_{A^{b}}$ is the kernel of the augmentation $\varepsilon_{A^{b}}: A^{b} \rightarrow R$. After choosing an epimorphism $j: R[\underline{X}] \rightarrow A^{b}$ locally on $\operatorname{Spec} R$, we have $I_{A^{b}}=$ $I_{R[\underline{X}]} /\left(I \cdot I_{R[\underline{X}]}\right)$, which implies $N_{\mathcal{H}}=I /\left(I \cdot I_{R[\underline{X}]}\right)$ and $t_{\mathcal{H}}^{*}=I_{R[\underline{X}]} / I_{R[\underline{X}]}^{2}$. Both are finite locally free $R$-modules of the same rank. This is obvious for $t_{\mathcal{H}}^{*}$, and for $N_{\mathcal{H}}$ a proof can be found in [HS, Lemma 3.3]. Also note that $I_{A^{b}} \cdot \operatorname{ker} i_{\mathcal{A}}=0$, because $\operatorname{ker} i_{\mathcal{A}}=I /\left(I \cdot I_{R[\underline{X}]}\right)$. We write $n=n_{\mathcal{H}}: N_{\mathcal{H}} \hookrightarrow A^{b}$ for the natural inclusion and $\pi=\pi_{\mathcal{H}}:=\left(\mathrm{id}-l_{A^{b}} \varepsilon_{A^{b}}\right) \bmod I_{A^{b}}^{2}: A^{b} \rightarrow t_{\mathcal{H}}^{*}$. If $\mathcal{H}=\left(A, A^{b}\right)$ and $\widetilde{\mathcal{H}}=\operatorname{Spec}\left(\widetilde{A}, \widetilde{A}^{b}\right)$, every morphism $\left(f, f^{b}\right):\left(A, A^{b}\right) \rightarrow\left(\widetilde{A}, \widetilde{A}^{b}\right)$ in $\operatorname{Hom}_{\mathrm{DSch}}(\widetilde{\mathcal{H}}, \mathcal{H})$ induces morphisms of $R$-modules $N_{f}: N_{\mathcal{H}} \rightarrow N_{\widetilde{\mathcal{H}}}$ and $t_{f}^{*}: t_{\mathcal{H}}^{*} \rightarrow t_{\widetilde{\mathcal{H}}}^{*}$ with $f^{b} \circ n_{\mathcal{H}}=n_{\widetilde{\mathcal{H}}} \circ N_{f}$ and $\pi_{\widetilde{\mathcal{H}}} \circ f^{b}=t_{f}^{*} \circ \pi_{\mathcal{H}}$.

Let $\mathcal{H}=\operatorname{Spec}\left(A, A^{b}, i_{\mathcal{A}}\right)$ and $\widetilde{\mathcal{H}}=\operatorname{Spec}\left(\widetilde{A}, \widetilde{A}^{b}, i_{\widetilde{\mathcal{A}}}\right)$ be objects in $\mathrm{DSch}_{S}$ and let $f: A \rightarrow \widetilde{A}$ be a morphism of augmented $R$-algebras. Faltings [Fal02, \$2] noted that the set

$$
\begin{aligned}
\mathcal{L}:=\left\{f^{b}: A^{b} \rightarrow \widetilde{A}^{b}\right. & \text { morphisms of augmented } R \text {-algebras for which } \\
& \left.\left(f, f^{b}\right) \in \operatorname{Hom}_{\mathrm{DSch}_{s}}(\widetilde{\mathcal{H}}, \mathcal{H})\right\}
\end{aligned}
$$

is non-empty and is a principal homogeneous space under $\operatorname{Hom}_{R}\left(t_{\mathcal{H}}^{*}, N_{\widetilde{\mathcal{H}}}\right)$. That is, for any $f^{b} \in \mathcal{L}$ the map $\operatorname{Hom}_{R}\left(t_{\mathcal{H}}^{*}, N_{\widetilde{\mathcal{H}}}\right) \rightarrow \mathcal{L}, h \mapsto f^{b}+n_{\widetilde{\mathcal{H}}} \circ h \circ \pi_{\mathcal{H}}$ is a bijection. For a proof, see [HS, Lemma 3.4] in the expanded version of this article on arXiv [HS].

The category $\operatorname{DSch}_{S}$ possesses direct products. If $\mathcal{H}=\operatorname{Spec}\left(A, A^{b}, i_{\mathcal{A}}\right)$ and $\widetilde{\mathcal{H}}=$ $\operatorname{Spec}\left(\widetilde{A}, \widetilde{A}^{b}, i_{\widetilde{\mathcal{A}}}\right)$, then the product $\mathcal{H} \times_{S} \widetilde{\mathcal{H}}$ is given by $\operatorname{Spec}\left(A \otimes_{R} \widetilde{A},\left(A \otimes_{R} \widetilde{A}\right)^{b}, \kappa\right)$, where

$$
(A \underset{R}{\otimes} \widetilde{A})^{b}:=\left(A_{R}^{b} \underset{R}{\otimes} \widetilde{A}^{b}\right) /\left(\operatorname{ker} i_{\mathcal{A}} \otimes \widetilde{A}^{b}+A^{b} \otimes \operatorname{ker} i_{\widetilde{\mathcal{A}}}\right) \cdot\left(I_{A^{b}} \otimes \widetilde{A}^{b}+A^{b} \otimes I_{\widetilde{A}^{b}}\right)
$$

and $\kappa$ is the natural epimorphism $\left(A \otimes_{R} A\right)^{b} \rightarrow A \otimes_{R} A$. After choosing locally on $\operatorname{Spec} R$ presentations $A=R[\underline{X}] / I, A^{b}=R[\underline{X}] /\left(I \cdot I_{R[\underline{X}]}\right)$, and $\widetilde{A}=R[\underline{\widetilde{X}}] / \tilde{I}, \widetilde{A}^{b}=$ $R[\underline{\widetilde{X}}] /\left(\tilde{I} \cdot I_{R[\underline{\widetilde{X}}]}\right)$, we can write

$(A \underset{R}{\otimes} \widetilde{A})^{b}=R[\underline{X} \otimes 1,1 \otimes \underline{\widetilde{X}}] /(I \otimes R[\underline{\widetilde{X}}]+R[\underline{X}] \otimes \tilde{I}) \cdot\left(I_{R[\underline{X}]} \otimes R[\underline{\widetilde{X}}]+R[\underline{X}] \otimes I_{R[\underline{\widetilde{X}}]}\right)$.

Definition 3.3 Let $\mathrm{DGr}_{S}$ be the category of group objects in $\mathrm{DSch}_{S}$. If $\mathcal{G}=\operatorname{Spec} \mathcal{A} \epsilon$ $\mathrm{DGr}_{S}$, then its group structure is given via the comultiplication $\Delta: A \rightarrow A \otimes_{R} A$ and $\Delta^{b}: A^{b} \rightarrow\left(A \otimes_{R} A\right)^{b}$, the counit $\varepsilon: A \rightarrow R$ and $\varepsilon^{b}: A^{b} \rightarrow R$, and the coinversion [-1]:A $\rightarrow$ $A$ and $[-1]^{b}: A^{b} \rightarrow A^{b}$, which satisfy the usual axioms. In particular, we require the 
counit axiom $\left(\operatorname{id}_{A^{b}} \otimes \varepsilon^{b}\right) \circ \Delta^{b}=\operatorname{id}_{A^{b}}=\left(\varepsilon^{b} \otimes \operatorname{id}_{A^{b}}\right) \circ \Delta^{b}$, and that $\varepsilon$ and $\varepsilon^{b}$ are the augmentation maps. The morphisms in $\mathrm{DGr}_{S}$ are morphisms of group objects.

If $\mathcal{G}=\left(G, G^{b}\right) \in \operatorname{DGr}_{S}$, note that $G=\operatorname{Spec} A$ is a finite locally free group scheme over $R$ with the comultiplication $\Delta$, the counit $\varepsilon$, and the coinversion [-1]. But, in general, $G^{b}$ is not a group scheme over $S$ when the comultiplication $\Delta^{b}: A^{b} \rightarrow\left(A \otimes_{R} A\right)^{b}$ does not lift to $A^{b} \otimes_{R} A^{b}$. Faltings and Abrashkin [Abr06, §1.2] made the following remarks.

(a) If $\mathcal{G}=\operatorname{Spec}\left(A, A^{b}, i_{\mathcal{A}}\right) \in \operatorname{DSch}_{S}$ and $G=\operatorname{Spec} A$ is a finite locally free group scheme over $R$, then there exists a unique structure of a group object on $\mathcal{G}$ that is compatible with that of $G$. It satisfies $\Delta^{b}(x)-x \otimes 1-1 \otimes x \in I_{A^{b}} \otimes I_{A^{b}}$ for all $x \in I_{A^{b}}$.

(b) If $\mathcal{G}, \mathcal{H} \in \mathrm{DGr}_{S}$ are group objects and $\left(f, f^{b}\right) \in \operatorname{Hom}_{\mathrm{DSch}}(\mathcal{G}, \mathcal{H})$ such that $f: G \rightarrow H$ is a morphism of group schemes, then $\left(f, f^{b}\right) \in \operatorname{Hom}_{\mathrm{DGr}_{s}}(\mathcal{G}, \mathcal{H})$.

See [HS, p. 10] for a proof.

Let $\mathcal{G}=\left(G, G^{b}, i_{\mathcal{G}}\right) \in \mathrm{DGr}_{S}$. Faltings defined the co-Lie complex of $\mathcal{G}$ over $S=$ $\operatorname{Spec} R$ (that is, the fiber at the unit section of $G$ of the cotangent complex) as the complex of finite locally free $R$-modules

$$
\dot{\ell_{\mathcal{G} / S}}: \quad 0 \longrightarrow N_{\mathcal{G}} \stackrel{d}{\longrightarrow} t_{\mathcal{G}}^{*} \longrightarrow 0
$$

concentrated in degrees -1 and 0 with differential $d:=\pi_{\mathcal{G}} \circ n_{\mathcal{G}}$. Recall that the co-Lie complex of $G / S$ and more generally the cotangent complex of a morphism were defined by Illusie [Ill71,Ill72] generalizing earlier work of Lichtenbaum and Schlessinger [LS67]. If $G=\operatorname{Spec} A$ for $A=R[\underline{X}] / I$, where $I$ is generated by a regular sequence, then the cotangent complex of Illusie [Ill71, II.1.2.3] is quasi-isomorphic to the complex of finite locally free $A$-modules

$$
\stackrel{\bullet}{L_{G / S}}: \quad 0 \longrightarrow I / I^{2} \stackrel{d}{\longrightarrow} \Omega_{R[\underline{X}] / R}^{1} \underset{R[\underline{X}]}{\otimes} A \longrightarrow 0
$$

concentrated in degrees -1 and 0 with $d$ being the differential map [Ill71, Corollaire III.3.2.7]. The co-Lie complex of $G$ over $S$ was defined by Illusie [Ill72, §VII.3.1] as $\ell_{G / S}^{\bullet}:=\varepsilon_{G}^{*} \dot{L}_{G / S}^{\bullet}$, where $\varepsilon_{G}: S \rightarrow G$ is the unit section. To see that this is equal to Faltings's definition note that

$$
\begin{gathered}
\varepsilon_{G}^{*}\left(I / I^{2}\right)=I / I^{2} \underset{A}{\otimes} R=I \underset{R[\underline{X}]}{\otimes} R=I /\left(I \cdot I_{R[\underline{X}]}\right)=N_{\mathcal{G}}, \\
\varepsilon_{G}^{*}\left(\Omega_{R[\underline{X}] / R}^{1} \underset{R[\underline{X}]}{\otimes} A\right)=\Omega_{R[\underline{X}] / R}^{1} \underset{R[\underline{X}]}{\otimes} R=\bigoplus_{v=1}^{n} R \cdot d X_{v}=I_{R[\underline{X}]} / I_{R[\underline{X}]}^{2}=t_{\mathcal{G}}^{*},
\end{gathered}
$$

and that the differential of both co-Lie complexes sends an element $x \in I$ to the linear term in its expansion as a polynomial in $\underline{X}$, because all terms of higher degree are sent to zero under $\varepsilon_{G}^{*}$.

Up to homotopy equivalence both ${\dot{L_{G / S}}}^{\bullet}$ and $\ell_{G / S}^{\bullet}$ only depend on $G$, and not on the presentation $A=R[\underline{X}] / I$ nor on the deformation $\mathcal{G}$ of $G$. Note that $\dot{L}_{G / S}^{\bullet}$ and $\iota^{*} \ell_{G / S}$ are quasi-isomorphic by [Mes72, Chapter II, Proposition 3.2.9], where $\iota: G \rightarrow S$ is the structure map. 
Definition 3.4 We (re-)define the co-Lie module of G over $S$ as $\omega_{G}:=\mathrm{H}^{0}\left(\ell_{\mathcal{G} / S}\right):=$ coker $d$ and set $n_{G}:=\mathrm{H}^{-1}\left(\ell_{\mathcal{G} / S}^{\bullet}\right):=\operatorname{ker} d$. These $R$-modules only depend on $G$ and not on $\mathcal{G}$. Since $\mathrm{H}^{0}\left(\dot{L}_{G / S}^{*}\right)=\Omega_{G / S}^{1}$, we have $\omega_{G}=\varepsilon_{G}^{*} \Omega_{G / S}^{1}$, which is also canonically isomorphic to the $R$-module of invariant differentials on $G$.

We record the following lemmas.

Lemma 3.5 If $\mathcal{G} \in \mathrm{DGr}_{S}$, the following are equivalent:

(i) $G$ is étale over $S$;

(ii) $\omega_{G}=0$;

(iii) the differential of $\dot{\ell}_{\mathcal{G} / S}^{\bullet}$ is an isomorphism.

Proof If $G$ is étale, then $\Omega_{G / S}^{1}=0$. Conversely, since $\Omega_{G / S}^{1}$ is a finitely generated $\mathcal{O}_{G}$-module, by Nakayama $\omega_{G}=0$ implies that $G$ is étale along the zero section. Being a group scheme, it is étale everywhere.

Clearly (iii) implies (ii). Conversely if $\omega_{G}=0$, that is, if $d$ is surjective, then $d$ is also injective, because both $t_{\mathcal{G}}^{*}$ and $N_{\mathcal{G}}$ are finite locally free of the same rank.

Lemma 3.6 ([Mes72, Chapter II, Proposition 3.3.4]) Let $0 \rightarrow G^{\prime} \rightarrow G \rightarrow G^{\prime \prime} \rightarrow 0$ be an exact sequence of finite locally free group schemes over $S$. Then there is an exact sequence of $R$-modules

$$
0 \longrightarrow n_{G^{\prime \prime}} \longrightarrow n_{G} \longrightarrow n_{G^{\prime}} \longrightarrow \omega_{G^{\prime \prime}} \longrightarrow \omega_{G} \longrightarrow \omega_{G^{\prime}} \longrightarrow 0 .
$$

\section{Strict $\mathbb{F}_{q}$-module Schemes}

We keep the notation of the previous section. Let $\mathcal{O}$ be a commutative unitary ring.

Definition 4.1 In this article an $\mathcal{O}$-module scheme over $S$ is a finite locally free commutative group scheme $G$ over $S$ together with a ring homomorphism $\mathcal{O} \rightarrow \operatorname{End}_{S}(G)$. We denote the category of $\mathcal{O}$-module schemes over $S$ by $\operatorname{Gr}(\mathcal{O})_{s}$.

Proposition 4.2 If $S$ is the spectrum of a field $L$, every $\mathcal{O}$-module scheme $G$ over $S$ is canonically an extension $0 \rightarrow G^{0} \rightarrow G \rightarrow G^{\text {ét }} \rightarrow 0$ of an étale $\mathcal{O}$-module scheme $G^{\text {ét }}$ by a connected $\mathcal{O}$-module scheme $G^{0}$. The $\mathcal{O}$-module scheme $G^{\text {ét }}$ is the largest étale quotient of $G$. If $L$ is perfect, $G^{\text {ét }}$ is canonically isomorphic to the reduced closed $\mathcal{O}$-module subscheme $G^{\text {red }}$ of $G$ and the extension splits canonically, $G=G^{0} \times_{S} G^{\text {red }}$.

Proof The constituents of the canonical decomposition of the finite $S$-group scheme $G$ are $\mathcal{O}$-invariant.

Definition 4.3 Let $S=\operatorname{Spec} R$ be a scheme over $\mathcal{O}$ and let $\mathcal{G} \in \mathrm{DGr}_{s}$. A strict $\mathcal{O}$-action on $\mathcal{G}$ is a homomorphism $\mathcal{O} \rightarrow \operatorname{End}_{\mathrm{DGr}_{\mathcal{S}}}(\mathcal{G})$ such that the induced action on $\dot{\ell}_{\mathcal{G} / \mathrm{S}}$ is equal to the scalar multiplication via $\mathcal{O} \rightarrow R$; $c f$ Remark 4.4.

We let $\operatorname{DGr}(\mathcal{O})_{S}$ be the category whose objects are pairs $(\mathcal{G},[\cdot])$ where $\mathcal{G} \in \mathrm{DGr}_{S}$ and $[\cdot]: \mathcal{O} \rightarrow \operatorname{End}_{\mathrm{DGr}_{S}}(\mathcal{G}), a \mapsto[a]$ is a strict $\mathcal{O}$-action, and whose morphisms 
$f:(\mathcal{G},[\cdot]) \rightarrow\left(\mathcal{G}^{\prime},[\cdot]^{\prime}\right)$ are those morphisms $f: \mathcal{G} \rightarrow \mathcal{G}^{\prime}$ in $\mathrm{DGr}_{S}$ that are compatible with the $\mathcal{O}$-actions, that is, they satisfy $f \circ[a]=[a]^{\prime} \circ f$ for all $a \in \mathcal{O}$.

We let $\operatorname{DGr}^{*}(\mathcal{O})_{s}$ be the quotient category of $\operatorname{DGr}(\mathcal{O})_{s}$ having the same objects, whose morphisms are the equivalence classes of morphisms $\left(G, G^{b}\right) \rightarrow\left(H, H^{b}\right)$ in $\operatorname{DGr}(\mathcal{O})_{S}$ that induce the same morphism $G \rightarrow H$.

So by definition the forgetful functor $\operatorname{DGr}^{*}(\mathcal{O})_{S} \rightarrow \operatorname{Gr}(\mathcal{O})_{S}$, which sends $\left(G, G^{b}\right)$ to $G$ and morphisms $\left(G, G^{b}\right) \rightarrow\left(H, H^{b}\right)$ to their restriction to $G \rightarrow H$, is faithful.

Faltings [Fal02, Remark b) after Definition 1] remarked that a strict $\mathcal{O}$-action [ $\cdot$ ] on $\mathcal{G}$ induces on every deformation $\widetilde{\mathcal{G}}$ of $G$ a unique strict $\mathcal{O}$-action $\widetilde{[\cdot]}$ that is compatible with all lifts $\widetilde{\mathcal{G}} \rightarrow \mathcal{G}$ and $\mathcal{G} \rightarrow \widetilde{\mathcal{G}}$ of the identity on $G$; for details see [HS, Lemma 4.4]. In particular, the pairs $(\mathcal{G},[\cdot])$ and $(\widetilde{\mathcal{G}}, \widetilde{[\cdot]})$ are isomorphic in $\operatorname{DGr}^{*}(\mathcal{O})_{S}$.

Remark 4.4 The co-Lie complex $\dot{\ell}_{\mathcal{G} / S}$ depends on the deformation $\mathcal{G}$ of $G$. For another deformation $\widetilde{\mathcal{G}}$ the complex $\dot{\ell}_{\widetilde{\mathcal{G}} / S}^{\bullet}$ is homotopically equivalent to $\ell_{\mathcal{G} / S}^{\bullet}$. Therefore one might try to weaken Definition 4.3 and only require that the action of $a \in \mathcal{O}$ on $\dot{\ell}_{\mathcal{G} / S}$ is homotopic to the scalar multiplication with $a$. We do not know whether this is equivalent to Definition 4.3 and whether Faltings's remark remains valid for general $\mathcal{O}$. Both are true for the polynomial ring $\mathcal{O}=\mathbb{F}_{p}[a]$.

Remark 4.5 Note that there can be different non-isomorphic strict $\mathcal{O}$-actions on a deformation 9 . For example, let $G=\boldsymbol{\alpha}_{p}=\operatorname{Spec} R[X] /\left(X^{p}\right)$ and $A^{b}=R[X] /\left(X^{p+1}\right)$. Let $\mathcal{O}=\mathbb{F}_{p}[a]$ be the polynomial ring in the variable $a$, and let $R$ be an $\mathcal{O}$-algebra by sending $a$ to 0 in $R$. For every $u \in R$ the endomorphism $[a]=0: \boldsymbol{\alpha}_{p} \rightarrow \boldsymbol{\alpha}_{p}, X \mapsto 0$ lifts to $[a]: A^{b} \rightarrow A^{b}, X \mapsto u X^{p}$. All these lifts define strict $\mathcal{O}$-actions on $\left(G\right.$, $\left.\operatorname{Spec} A^{b}\right)$ that are non-isomorphic in $\mathrm{DGr}^{*}\left(\mathbb{F}_{p}[a]\right)_{s}$. In particular, the forgetful functor

$$
\operatorname{DGr}^{*}\left(\mathbb{F}_{p}[a]\right)_{S} \rightarrow \operatorname{Gr}\left(\mathbb{F}_{p}[a]\right)_{S}
$$

is not fully faithful.

In contrast, for $\mathcal{O}=\mathbb{F}_{q}$ we have the following lemma.

Lemma 4.6 The forgetful functor $\mathrm{DGr}^{*}\left(\mathbb{F}_{q}\right)_{S} \rightarrow \mathrm{Gr}\left(\mathbb{F}_{q}\right)_{S}$ is fully faithful. In particular, if $G \in \operatorname{Gr}\left(\mathbb{F}_{q}\right)_{S}$ and $\mathcal{G}=\left(G, G^{b}\right) \in \mathrm{DGr}_{s}$ is a deformation of $G$, then there is at most one strict $\mathbb{F}_{q}$-action on $\mathcal{G}$ that lifts the action on $G$.

Proof Let $(\mathcal{G},[\cdot])$ and $(\widetilde{\mathcal{G}}, \widetilde{[\cdot]})$ be in $\operatorname{DGr}^{*}\left(\mathbb{F}_{q}\right)_{S}$ with $\mathcal{G}=\operatorname{Spec}\left(A, A^{b}\right)$ and $\widetilde{\mathcal{G}}=$ $\operatorname{Spec}\left(\widetilde{A}, \widetilde{A}^{b}\right)$. Let $f: A \rightarrow \widetilde{A}$ be a morphism in $\operatorname{Gr}\left(\mathbb{F}_{q}\right)_{s}$, that is $\widetilde{[a]} f=f[a]$. Take any lift $f^{b}: A^{b} \rightarrow \widetilde{A}^{b}$ of $f$. Then for each $a \in \mathbb{F}_{q}$ there is an $R$-homomorphism $h_{a}: t_{\mathcal{S}}^{*} \rightarrow N_{\widetilde{\mathcal{G}}}$ with $[\widetilde{a}] f^{b}-f^{b}[a]=\tilde{n} h_{a} \pi$. It satisfies $h_{a b}=a h_{b}+b h_{a}$ because $\pi[b]=t_{[b]}^{*} \pi=b \pi$ and $\widetilde{[a]} \tilde{n}=\tilde{n} N_{\widetilde{[a]}}=a \tilde{n}$, and hence

$$
\begin{aligned}
\tilde{n} h_{a b} \pi & =\widetilde{[a b]} f^{b}-f^{b}[a b]=\widetilde{[a]}\left(\widetilde{[b]} f^{b}-f^{b}[b]\right)+\left(\widetilde{[a]} f^{b}-f^{b}[a]\right)[b] \\
& =\widetilde{[a]} \tilde{n} h_{b} \pi+\tilde{n} h_{a} \pi[b]=\tilde{n}\left(a h_{b}+b h_{a}\right) \pi .
\end{aligned}
$$


We claim that it also satisfies $h_{a+b}=h_{a}+h_{b}$. Namely,

$$
\begin{aligned}
\tilde{n} h_{a+b} \pi & =\widetilde{[a+b]} f^{b}-f^{b}[a+b]=\widetilde{m} \circ\left(\widetilde{[a]} f^{b} \otimes \widetilde{[b]} f^{b}-f^{b}[a] \otimes f^{b}[b]\right) \circ \Delta^{b} \\
& =\widetilde{m} \circ\left(\tilde{n} h_{a} \pi \otimes \widetilde{[b]} f^{b}+f^{b}[a] \otimes \tilde{n} h_{b} \pi\right) \circ \Delta^{b},
\end{aligned}
$$

where $\widetilde{m}:\left(\widetilde{A} \otimes_{R} \widetilde{A}\right)^{b} \rightarrow \widetilde{A}^{b}$ is induced from the multiplication in the ring $\widetilde{A}^{b}$ and $\left(\widetilde{[a]} f^{b} \otimes \widetilde{[b]} f^{b}\right): A^{b} \otimes_{R} A^{b} \rightarrow \widetilde{A^{b}} \otimes_{R} \widetilde{A}^{b}$ induces a homomorphism

$$
\left.\underset{R}{(A \otimes} A)^{b} \rightarrow \underset{R}{\widetilde{A} \otimes} \underset{A}{\otimes}\right)^{b},
$$

denoted by the same symbol. We evaluate this expression on $X_{v}$, where

$$
A^{b}=R[\underline{X}] / I \cdot I_{R[\underline{X}} .
$$

For every $v$ there are $u_{i}, v_{i} \in I_{R[X]}$ such that $\Delta^{b}\left(X_{v}\right)=X_{v} \otimes 1+1 \otimes X_{v}+\sum_{i} u_{i} \otimes v_{i}$; see (a) after Definition 3.3. Now $\pi(1)=0$, together with $\left(\tilde{n} \tilde{h}_{a} \pi\right)\left(I_{R[\underline{X}]}\right) \subset \tilde{I} / \tilde{I} \cdot I_{R[\underline{\widetilde{X}}]} \subset \widetilde{A}^{b}$ and $\widetilde{[b]} f^{b}\left(I_{R[\underline{X}]}\right) \subset I_{R[\underline{X}]}$ imply $\tilde{n} h_{a+b} \pi\left(X_{v}\right)=\tilde{n} h_{a} \pi\left(X_{v}\right)+\tilde{n} h_{b} \pi\left(X_{v}\right)$ as desired. This proves $h_{a+b}=h_{a}+h_{b}$. If $a$ lies in the image $\mathbb{F}_{p}$ of $\mathbb{Z}$ in $\mathbb{F}_{q}$, then $h_{a}=a \cdot h_{1}=0$. In other words $a \mapsto h_{a}, \mathbb{F}_{q} \rightarrow \operatorname{Hom}_{R}\left(t_{\mathcal{G}}^{*}, N_{\widetilde{\mathcal{G}}}\right)$ is an $\mathbb{F}_{p}$-derivation. Since $\Omega_{\mathbb{F}_{q} / \mathbb{F}_{p}}^{1}=(0)$, we must have $h_{a}=0$ and $\widetilde{[a]} f^{b}=f^{b}[a]$ for all $a \in \mathbb{F}_{q}$. This means that $\left(f, f^{b}\right)$ defines a morphism in $\operatorname{DGr}^{*}\left(\mathbb{F}_{q}\right)_{S}$ that maps to $f$ under the forgetful functor. So this functor is fully faithful. The remaining assertion follows by taking $\widetilde{A}^{b}=A^{b}, \widetilde{A}=A$ and $f^{b}=\mathrm{id}$.

Definition 4.7 A finite locally free $\mathbb{F}_{q}$-module scheme $G$ over $R$ is called a strict $\mathbb{F}_{q}$-module scheme if it lies in the essential image of the forgetful functor $\operatorname{DGr}^{*}\left(\mathbb{F}_{q}\right)_{S} \rightarrow \operatorname{Gr}\left(\mathbb{F}_{q}\right)_{S}$, that is, if it has a deformation $\mathcal{G}$ carrying a strict $\mathbb{F}_{q}$-action that lifts the $\mathbb{F}_{q}$-action on $G$. We identify $\operatorname{DGr}^{*}\left(\mathbb{F}_{q}\right)_{S}$ with the category of finite locally free strict $\mathbb{F}_{q}$-module schemes over $S$.

Lemma 4.8 For a finite locally free $\mathbb{F}_{q}$-module scheme $G$ over $R$, the property of being a strict $\mathbb{F}_{q}$-module scheme is local on Spec $R$.

Proof Let $\widetilde{\mathcal{G}}$ be a deformation of $G$ over $\operatorname{Spec} R$. Let $\operatorname{Spec} R_{i} \subset \operatorname{Spec} R$ be an open covering and let $\mathcal{G}_{i}$ be deformations of $G \times{ }_{R} \operatorname{Spec} R_{i}$ carrying a strict $\mathbb{F}_{q}$-action which lifts the $\mathbb{F}_{q}$-action on $G$. This action induces by [Fal02, Remark b) after Definition 1] a strict $\mathbb{F}_{q}$-action on $\widetilde{\mathcal{G}} \times{ }_{R} \operatorname{Spec} R_{i}$ for all $i$. Above $\operatorname{Spec} R_{i} \cap \operatorname{Spec} R_{j}$ these actions coincide by Lemma 4.6 , and hence they glue to a strict $\mathbb{F}_{q}$-action on $\widetilde{\mathcal{G}}$ as desired.

Example 4.9 We give examples for finite locally free strict $\mathbb{F}_{q}$-module schemes. Let $R$ be an $\mathbb{F}_{q}$-algebra.

(a) Let $\boldsymbol{\alpha}_{q}=\operatorname{Spec} R[X] /\left(X^{q}\right)$ and $\boldsymbol{\alpha}_{q}^{b}=\operatorname{Spec} R[X] /\left(X^{q+1}\right)$. Then $[a](X)=a X$ for $a \in \mathbb{F}_{q}$ defines a strict $\mathbb{F}_{q}$-action on $\mathcal{G}=\left(\boldsymbol{\alpha}_{q}, \boldsymbol{\alpha}_{q}^{b}\right)$. Indeed, the co-Lie complex is

$$
\ell_{\mathcal{G} / S}: \quad 0 \longrightarrow X^{q} \cdot R \longrightarrow X \cdot R \longrightarrow 0
$$


with $d=0$ and $a \in \mathbb{F}_{q}$ acts on it as scalar multiplication by $a$ because $N_{[a]}\left(X^{q}\right)=$ $(a X)^{q}=a X^{q}$ and $t_{[a]}^{*}(X)=a X$. Therefore $\boldsymbol{\alpha}_{q}$ is a finite locally free strict $\mathbb{F}_{q}$-module scheme.

(b) On $\boldsymbol{\alpha}_{p}=\operatorname{Spec} R[X] /\left(X^{p}\right)$ there is an $\mathbb{F}_{q}$-action given by $[a](X)=a X$. If $q \neq p$, it does not lift to a strict $\mathbb{F}_{q}$-action on $\boldsymbol{\alpha}_{p}^{b}=\operatorname{Spec} R[X] /\left(X^{p+1}\right)$. Although we may lift the action to $\mathcal{G}=\left(\boldsymbol{\alpha}_{p}, \boldsymbol{\alpha}_{p}^{b}\right)$ via $[a](X)=a X$, the co-Lie complex is

$$
\ell_{\mathcal{G} / S}: \quad 0 \longrightarrow X^{p} \cdot R \longrightarrow X \cdot R \longrightarrow 0
$$

and so $a \in \mathbb{F}_{q}$ acts on $N_{\mathcal{G}}$ by $a^{p}$ which is not scalar multiplication by $a$ when $a^{p} \neq a$. Any other lift $\widetilde{[a]}$ of the $\mathbb{F}_{q}$-action on $\boldsymbol{\alpha}_{p}$ to $\mathcal{G}$ satisfies $\widetilde{[a]}=[a]+n h_{a} \pi$ for an $R$-homomorphism $h_{a}: t_{\mathcal{G}}^{*} \rightarrow N_{\mathcal{G}}$ and yields $n N_{\widetilde{[a]}}=\widetilde{[a]} n=[a] n+n h_{a} \pi n=[a] n=n N_{[a]}$ because $\pi n=d=0$ on $\ell_{\mathcal{G} / S}^{\bullet}$. So no such action is strict and $\boldsymbol{\alpha}_{p}$ is not a strict $\mathbb{F}_{q^{-}}$ module scheme.

(c) The constant étale group scheme $\mathbb{F}_{q}=\operatorname{Spec} R[X] /\left(X^{q}-X\right)$ over $\operatorname{Spec} R$ and its deformation $\mathbb{F}_{q}{ }^{b}=\operatorname{Spec} R[X] /\left(X^{q+1}-X^{2}\right)$ carry a strict $\mathbb{F}_{q}$-action via $[a](X)=$ $a X$. Indeed, the co-Lie complex is

$$
\ell_{\mathcal{G} / S}: \quad 0 \longrightarrow\left(X-X^{q}\right) \cdot R \longrightarrow X \cdot R \longrightarrow 0
$$

with $d: X-X^{q} \mapsto X$ and $a \in \mathbb{F}_{q}$ acts on it by $N_{[a]}\left(X-X^{q}\right)=a X-(a X)^{q}=a\left(X-X^{q}\right)$ and $t_{[a]}^{*}(X)=a X$. Therefore $\mathbb{F}_{q}$ is a finite locally free strict $\mathbb{F}_{q}$-module scheme.

(d) The multiplicative group $\boldsymbol{\mu}_{p}=\operatorname{Spec} R[X] /\left(X^{p}-1\right)$ has an $\mathbb{F}_{p}$-action via

$$
[a](X)=X^{a} \text {. }
$$

This action does not lift to $\mu_{p}^{b}=\operatorname{Spec} R[X] /(X-1)^{p+1}$, because on $\mu_{p}^{b}$ we have $\Delta(X)=$ $X \otimes X$ and hence $[a](X)=X^{a}$, which satisfies $[p](X)=X^{p} \neq 1$. Therefore no deformation of $\boldsymbol{\mu}_{p}$ can carry a strict $\mathbb{F}_{p}$-action and $\boldsymbol{\mu}_{p}$ is not a strict $\mathbb{F}_{p}$-module scheme. Note that nevertheless $\mathbb{F}_{p}$ acts through scalar multiplication on the co-Lie complex $\ell_{\boldsymbol{\mu}_{p} / S}^{\bullet}$

Part (c) generalizes to the following lemma.

Lemma 4.10 Any finite étale $\mathbb{F}_{q}$-module scheme is a finite locally free strict $\mathbb{F}_{q}$-module. In particular, if $0 \rightarrow G^{\prime} \rightarrow G \rightarrow G^{\prime \prime} \rightarrow 0$ is an exact sequence of finite locally free $\mathbb{F}_{q}$-module schemes with $G$ a strict $\mathbb{F}_{q}$-module and $G^{\prime \prime}$ étale, then both $G^{\prime}$ and $G^{\prime \prime}$ are strict $\mathbb{F}_{q}$-modules.

Proof The first assertion was remarked by Faltings [Fal02, §3, p. 252] more generally for finite étale $\mathcal{O}$-module schemes, and also follows from [Dri87, Proposition 2.1(6)] and Theorem 5.2 below. (For a direct proof, see [HS, Lemma 4.11].) The last assertion on the strictness of $G^{\prime}$ can be proved on affine open subsets of $S$. There Lemma 4.6 implies that the morphism $G \rightarrow G^{\prime \prime}$ is $\mathbb{F}_{q}$-strict in the sense of Faltings [Fal02, Definition 1], and by [Fal02, Proposition 2] its kernel $G^{\prime}$ is a strict $\mathbb{F}_{q}$-module. 


\section{Equivalence Between Finite $\mathbb{F}_{q}$-shtukas and Strict $\mathbb{F}_{q}$-modules}

Let $S$ be a scheme over Spec $\mathbb{F}_{q}$. Recall that a finite locally free commutative group scheme $G$ over $S$ is equipped with a relative $p$-Frobenius $F_{p, G}: G \rightarrow \sigma_{p}^{*} G$ and a $p$-Verschiebung morphism $V_{p, G}: \sigma_{p}^{*} G \rightarrow G$ that satisfy

$$
F_{p, G} \circ V_{p, G}=p \operatorname{id}_{\sigma_{p}^{*} G} \text { and } V_{p, G} \circ F_{p, G}=p \operatorname{id}_{G} .
$$

For more details see [SGA 3, Exposé VII $_{\mathrm{A}}, \$ 4.3$ ]. Example 4.9 is generalized by the following results of Abrashkin. The first is concerned with finite locally free strict $\mathbb{F}_{p}$-module schemes.

Theorem 5.1 ( [Abr06, Theorem 1]) Let G be a finite locally free group scheme equipped with an $\mathbb{F}_{p}$-action over an $\mathbb{F}_{p}$-scheme $S$. Then this action lifts (uniquely) to a strict $\mathbb{F}_{p}$-action on some (any) deformation of $G$ if and only if the p-Verschiebung of $G$ is zero. In particular, the forgetful functor induces an equivalence between $\mathrm{DGr}^{*}\left(\mathbb{F}_{p}\right)_{S}$ and the category of those group schemes in $\operatorname{Gr}\left(\mathbb{F}_{p}\right)_{s}$ that have p-Verschiebung zero.

To explain Abrashkin's classification of finite locally free strict $\mathbb{F}_{q}$-module schemes we recall that Drinfeld [Dri87, \$2] defined a functor from finite $\mathbb{F}_{q}$-shtukas over $S$ to finite locally free $\mathbb{F}_{q}$-module schemes over $S$. Abrashkin [Abr06] proved that the essential image of Drinfeld's functor consists of finite locally free strict $\mathbb{F}_{q}$-module schemes. Other descriptions of the essential image were given by Taguchi [Tag95, \$1] and Laumon [Lau96, §B.3]. (But note that [Lau96, Propositions 2.4.11, B.3.13, and Lemma B.3.16] are incorrect as the $\mathbb{F}_{q}$-module scheme $G=\boldsymbol{\alpha}_{p}=\operatorname{Spec} R[x] /\left(x^{p}\right)$ shows when $p \neq q$.) Drinfeld's functor is defined as follows. Let $\underline{M}=\left(M, F_{M}\right)$ be a finite $\mathbb{F}_{q}$-shtuka over $S$. Let

$$
E=\underline{\operatorname{Spec}} \underline{S}_{n \geq 0}^{\oplus} \operatorname{Sym}_{\mathcal{O}_{s}}^{n} M
$$

be the geometric vector bundle corresponding to $M$, and let $F_{q, E}: E \rightarrow \sigma_{q}^{*} E$ be its relative $q$-Frobenius morphism over $S$. On the other hand, the map $F_{M}$ induces another $S$-morphism $\operatorname{Spec}\left(\operatorname{Sym}^{\bullet} F_{M}\right): E \rightarrow \sigma_{q}^{*} E$. Drinfeld defined

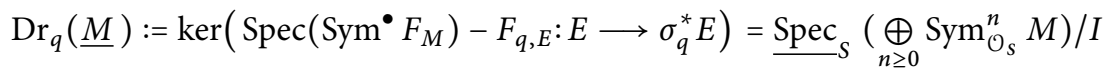

where the ideal $I$ is generated by the elements $m^{\otimes q}-F_{M}\left(\sigma_{q}^{*} m\right)$ for all local sections $m$ of $M$. (Here $m^{\otimes q}$ lives in $\operatorname{Sym}_{\mathcal{O}_{S}}^{q} M$ and $F_{M}\left(\sigma_{q}^{*} m\right)$ in $\operatorname{Sym}_{\mathcal{O}_{S}}^{1} M$.)

There is an equivalent description of $\operatorname{Dr}_{q}(\underline{M})$ as follows. Let $S=\operatorname{Spec} R$ be affine and denote the $R$-module $\Gamma(S, M)$ again by $M$. Let Frob $_{q, R}: R \rightarrow R$ be the $q$-Frobenius on $R$ with $x \mapsto x^{q}$. We equip $M$ with the Frob $_{q, R}$-semi-linear endomorphism

$$
F_{M}^{\text {semi }}: M \longrightarrow M, \quad m \longmapsto F_{M}\left(\sigma_{q}^{*} m\right),
$$

that satisfies

$$
F_{M}^{\mathrm{semi}}(b m)=F_{M}\left(\sigma_{q}^{*}(b m)\right)=F_{M}\left(b^{q} \sigma_{q}^{*} m\right)=b^{q} F_{M}^{\mathrm{semi}}(m) .
$$

Also we equip every $R$-algebra $T$ with the $\operatorname{Frob}_{q, R}$-semi-linear $R$-module endomorphism $F_{T}^{\text {semi }}:=\operatorname{Frob}_{q, T}: T \rightarrow T$. Then $\operatorname{Dr}_{q}(\underline{M})$ is the group scheme over $S$ that is 
given on $R$-algebras $T$ as

$$
\begin{aligned}
\operatorname{Dr}_{q}(\underline{M})(T) & =\operatorname{Hom}_{F^{\text {semi }}}(\underline{M}, T) \\
& :=\left\{h \in \operatorname{Hom}_{R-\operatorname{Mod}}(M, T): h(m)^{q}=h\left(F_{M}\left(\sigma_{q}^{*} m\right)\right) \forall m \in M\right\},
\end{aligned}
$$

because $\operatorname{Hom}_{R-\operatorname{Mod}}(M, T)=\operatorname{Hom}_{R-\operatorname{Alg}}\left(\operatorname{Sym}_{R}^{\bullet} M, T\right)=E(\operatorname{Spec} T){ }^{1}$

Now $\operatorname{Dr}_{q}(\underline{M})$ is an $\mathbb{F}_{q}$-module scheme over $S$ via the comultiplication $\Delta: m \mapsto$ $m \otimes 1+1 \otimes m$ and the $\mathbb{F}_{q}$-action $[a]: m \mapsto a m$ that it inherits from $E$. It has a canonical deformation

$$
\operatorname{Dr}_{q}(\underline{M})^{b}:=\underline{\operatorname{Spec}} S\left(\underset{n \geq 0}{\oplus} \operatorname{Sym}_{\mathcal{O}_{s}}^{n} M\right) /\left(I \cdot I_{0}\right),
$$

where $I_{0}=\bigoplus_{n \geq 1} \operatorname{Sym}_{\mathcal{O}_{s}}^{n} M$ is the ideal generated by all $m \in M$. This deformation is equipped with both the comultiplication $\Delta^{b}: m \mapsto m \otimes 1+1 \otimes m$ and the $\mathbb{F}_{q}$-action $[a]^{b}: m \mapsto a m$. We set $\mathcal{D r}_{q}(\underline{M}):=\left(\operatorname{Dr}_{q}(\underline{M}), \operatorname{Dr}_{q}(\underline{M})^{b}\right)$. Its co-Lie complex is

$$
0 \longrightarrow I /\left(I \cdot I_{0}\right) \longrightarrow I_{0} / I_{0}^{2} \longrightarrow 0
$$

with differential $d: m^{\otimes q}-F_{M}\left(\sigma_{q}^{*} m\right) \mapsto-F_{M}\left(\sigma_{q}^{*} m\right)$. On it [a] acts by scalar multiplication with $a$ because $(a m)^{q}-F_{M}\left(\sigma_{q}^{*}(a m)\right)=a^{q}\left(m^{\otimes q}-F_{M}\left(\sigma_{q}^{*} m\right)\right)$. This defines the functor $\mathcal{D} r_{q}: \mathbb{F}_{q}-\operatorname{Sht}_{S} \rightarrow \operatorname{DGr}\left(\mathbb{F}_{q}\right)_{S}$. We also compose $\mathcal{D} r_{q}$ with the projection to $\operatorname{DGr}^{*}\left(\mathbb{F}_{q}\right)_{S}$.

Conversely, let $\mathcal{G}=\left(G, G^{b}\right)=\operatorname{Spec}\left(A, A^{b}\right) \in \operatorname{DGr}\left(\mathbb{F}_{q}\right)_{S}$ in the affine situation $S=$ Spec $R$. Note that on the additive group scheme $\mathbb{G}_{a, S}=\operatorname{Spec} R[x]$ the elements $b \in R$ act via endomorphisms $\psi_{b}: \mathbb{G}_{a, S} \rightarrow \mathbb{G}_{a, S}$ given by $\psi_{b}^{*}: R[x] \rightarrow R[x], x \mapsto b x$. This makes $\mathbb{G}_{a, S}$ into an $R$-module scheme, and in particular, into an $\mathbb{F}_{q}$-module scheme via $\mathbb{F}_{q} \subset R$. We associate with $\mathcal{G}$ the $R$-module of $\mathbb{F}_{q}$-equivariant homomorphisms on $S$

$$
\begin{aligned}
M_{q}(\mathcal{G}) & :=\operatorname{Hom}_{R \text {-groups, }} \mathbb{F}_{q} \text {-lin }\left(G, \mathbb{G}_{a, S}\right) \\
& =\left\{x \in A: \Delta(x)=x \otimes 1+1 \otimes x,[a](x)=a x, \forall a \in \mathbb{F}_{q}\right\},
\end{aligned}
$$

with its action of $R$ via $R \rightarrow \operatorname{End}_{R \text {-groups, } \mathbb{F}_{q} \text {-lin }}\left(\mathbb{G}_{a, S}\right)$. It is a finite locally free $R$-module by [Pog17, Proposition 3.6, Remark 5.5]; see also [SGA 3, $\mathrm{VII}_{\mathrm{A}}, 7.4 .3$ ] in the reedited version of SGA 3 by P. Gille and P. Polo. The composition on the left with the relative $q$-Frobenius endomorphism $F_{q, \mathbb{G}_{a, S}}$ of $\mathbb{G}_{a, S}=\operatorname{Spec} R[x]$ given by $x \mapsto x^{q}$ defines a map $M_{q}(\mathcal{G}) \rightarrow M_{q}(\mathcal{G}), m \mapsto F_{q, \mathbb{G}_{a, s}} \circ m$ that is not $R$-linear, but $\sigma_{q}^{*}$-linear, because $F_{q, \mathbb{G}_{a, s}} \circ \psi_{b}=\psi_{b^{q}} \circ F_{q, \mathbb{G}_{a, S}}$. Therefore, $F_{q, \mathbb{G}_{a, S}}$ induces an $R$-homomorphism $F_{M_{q}(\mathcal{G})}: \sigma_{q}^{*} M_{q}(\mathcal{G}) \rightarrow M_{q}(\mathcal{G})$. Then $\underline{M}_{q}(\mathcal{G}):=\left(M_{q}(\mathcal{G}), F_{M_{q}(\mathcal{G})}\right)$ is a finite shtuka over $S$. Note that for $m \in M_{q}(\mathcal{G})$ the commutative diagram

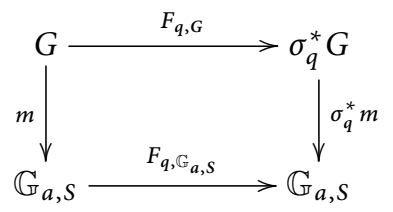

${ }^{1}$ We thank L. Taelman for mentioning this to us. 
implies that $F_{M_{q}(\mathcal{G})}\left(\sigma_{q}^{*} m\right):=F_{q, \mathbb{G}_{a, S}} \circ m=\sigma_{q}^{*} m \circ F_{q, G}$. If $\mathcal{H} \in \operatorname{DGr}\left(\mathbb{F}_{q}\right)_{S}$ and $\left(f, f^{b}\right): \mathcal{G} \rightarrow \mathcal{H}$ is a morphism in the category $\operatorname{DGr}\left(\mathbb{F}_{q}\right)_{s}$, then $\underline{M}_{q}(f): \underline{M}_{q}(\mathcal{H}) \rightarrow$ $\underline{M}_{q}(\mathcal{G}), m \mapsto m \circ f$. This defines the functor $\underline{M}_{q}: \operatorname{DGr}\left(\mathbb{F}_{q}\right)_{s} \rightarrow \mathbb{F}_{q}$-Sht ${ }_{s}$. It factors through the category $\operatorname{DGr}^{*}\left(\mathbb{F}_{q}\right)_{S}$ and further over the forgetful functor through the category of finite locally free strict $\mathbb{F}_{q}$-module schemes over $S$.

There is a natural morphism $\underline{M} \rightarrow \underline{M}_{q}\left(\operatorname{Dr}_{q}(\underline{M})\right), m \mapsto f_{m}$, where $f_{m}: \operatorname{Dr}_{q}(\underline{M}) \rightarrow$ $\mathbb{G}_{a, S}=\operatorname{Spec} R[x]$ is given by $f_{m}^{*}(x)=m$. There is also a natural morphism of group schemes $G \rightarrow \operatorname{Dr}_{q}\left(\underline{M}_{q}(G)\right)$ given on the structure sheaves by

$$
\underset{n \geq 0}{\oplus} \operatorname{Sym}_{\mathcal{O}_{s}}^{n} M_{q}(G) / I \rightarrow \mathcal{O}_{G}, m \mapsto m^{*}(x),
$$

which is well defined because

$$
F_{M_{q}(G)}\left(\sigma_{q}^{*} m\right)^{*}(x)=\left(F_{q, \mathbb{G}_{a, S}} \circ m\right)^{*}(x)=m^{*}\left(x^{q}\right)=\left(m^{*}(x)\right)^{q} .
$$

A large part of the following theorem was already proved by Drinfeld [Dri87, Proposition 2.1] without using the notion of strict $\mathbb{F}_{q}$-modules.

Theorem 5.2 (i) The contravariant functors $\operatorname{Dr}_{q}$ and $\underline{M}_{q}$ are mutually quasi-inverse anti-equivalences between the category of finite $\mathbb{F}_{q}$-shtukas over $S$ and the category of finite locally free strict $\mathbb{F}_{q}$-module schemes over $S$.

(ii) Both functors are $\mathbb{F}_{q}$-linear and map short exact sequences to short exact sequences. They preserve étale objects and map the canonical decompositions from Propositions 4.2 and 2.7 to each other.

(iii) Let $\underline{M}=\left(M, F_{M}\right)$ be a finite $\mathbb{F}_{q}$-shtuka over $S$ and let $G=\operatorname{Dr}_{q}(\underline{M})$. Then

(a) the natural morphisms

$$
\underline{M} \rightarrow \underline{M}_{q}\left(\operatorname{Dr}_{q}(\underline{M})\right), m \mapsto f_{m} \quad \text { and } \quad G \rightarrow \operatorname{Dr}_{q}\left(\underline{M}_{q}(G)\right)
$$

are isomorphisms;

(b) the $\mathbb{F}_{q}$-module scheme $\operatorname{Dr}_{q}(\underline{M})$ is radicial over $S$ if and only if $F_{M}$ is nilpotent locally on $S$;

(c) the order of the S-group scheme $\operatorname{Dr}_{q}(\underline{M})$ is $q^{\text {rkM}}$;

(d) the co-Lie complex $\hat{\ell}_{\mathcal{D} r_{q}(\underline{M}) / s}$ is canonically isomorphic to the complex

$$
0 \rightarrow \sigma_{q}^{*} M \stackrel{F_{M}}{\longrightarrow} M \rightarrow 0 .
$$

In particular, $\omega_{\operatorname{Dr}_{q}(\underline{M})}=\operatorname{coker} F_{M}$ and $n_{\operatorname{Dr}_{q}(\underline{M})}=\operatorname{ker} F_{M}$.

Proof Assertions (i) and (iii)(a) were proved by Abrashkin [Abr06, Theorem 2] in terms of the category $\mathrm{DGr}^{*}\left(\mathbb{F}_{q}\right)_{s}$.

(ii) The $\mathbb{F}_{q}$-linearity is clear from the definitions and the compatibility with étale objects follows from (iii)(d) and Lemma 3.5. Let $0 \rightarrow \underline{M}^{\prime \prime} \rightarrow \underline{M} \rightarrow \underline{M}^{\prime} \rightarrow 0$ be a short exact sequence of finite $\mathbb{F}_{q}$-shtukas. Then by construction $\operatorname{Dr}_{q}\left(\underline{M}^{\prime}\right) \rightarrow \operatorname{Dr}_{q}(\underline{M})$ is a closed immersion. Using (i), we consider the local sections of

$$
M^{\prime \prime}=\mathcal{H}_{\operatorname{Com}_{S} \text {-groups, } \mathbb{F}_{q} \text {-lin }}\left(\operatorname{Dr}_{q}\left(\underline{M}^{\prime \prime}\right), \mathbb{G}_{a, S}\right)
$$

that are obtained by the closed immersion $\operatorname{Dr}_{q}\left(\underline{M}^{\prime \prime}\right) \hookrightarrow \operatorname{Spec}_{S}\left(\operatorname{Sym}_{\mathcal{O}_{s}}^{\bullet} M^{\prime \prime}\right)$ composed with local coordinate functions on $\underline{\operatorname{Spec}}_{S}\left(\operatorname{Sym}_{\mathcal{O}_{s}}^{\bullet} M^{\prime \prime}\right)$. These local sections go 
to zero in $M^{\prime}$ and this yields a morphism $\operatorname{Dr}_{q}(\underline{M}) / \operatorname{Dr}_{q}\left(\underline{M}^{\prime}\right) \rightarrow \operatorname{Dr}_{q}\left(\underline{M}^{\prime \prime}\right)$. The latter must be an isomorphism by (i) due to the identification

$$
\begin{aligned}
\underline{M}_{q}\left(\operatorname{Dr}_{q}(\underline{M}) / \operatorname{Dr}_{q}\left(\underline{M}^{\prime}\right)\right) & =\operatorname{ker}\left(\underline{M}_{q}\left(\operatorname{Dr}_{q}(\underline{M})\right) \rightarrow \underline{M}_{q}\left(\operatorname{Dr}_{q}\left(\underline{M}^{\prime}\right)\right)\right) \\
& =\underline{M}^{\prime \prime}=\underline{M}_{q}\left(\operatorname{Dr}_{q}\left(\underline{M}^{\prime \prime}\right)\right) .
\end{aligned}
$$

Conversely let $0 \rightarrow G^{\prime} \rightarrow G \rightarrow G^{\prime \prime} \rightarrow 0$ be a short exact sequence of finite locally free strict $\mathbb{F}_{q}$-module schemes. Then the exactness of $0 \rightarrow \underline{M}_{q}\left(G^{\prime \prime}\right) \rightarrow \underline{M}_{q}(G) \rightarrow$ $\underline{M}_{q}\left(G^{\prime}\right)$ is obvious. Applying $\mathrm{Dr}_{q}$, whose exactness we just established, to the injection $\underline{M}_{q}(G) / \underline{M}_{q}\left(G^{\prime \prime}\right) \rightarrow \underline{M}\left(G^{\prime}\right)$ yields an isomorphism $\operatorname{Dr}_{q}\left(\underline{M}_{q}(G) / \underline{M}_{q}\left(G^{\prime \prime}\right)\right)=$ $\operatorname{ker}\left(G \rightarrow G^{\prime \prime}\right)=G^{\prime}$. From (i) it follows that $\underline{M}_{q}(G) / \underline{M}_{q}\left(G^{\prime \prime}\right) \rightarrow \underline{M}\left(G^{\prime}\right)$ is an isomorphism.

Consider the exact sequences from Propositions 4.2 and 2.7. $\operatorname{Then}^{\operatorname{Dr}}{ }_{q}\left(\underline{M}_{\text {ét }}\right)$ is an étale quotient of $\operatorname{Dr}_{q}(\underline{M})$. This yields a morphism $\operatorname{Dr}_{q}(\underline{M})^{\text {ét }} \rightarrow \operatorname{Dr}_{q}(\underline{M}$ ét $)$. Conversely, $\underline{M}_{q}\left(G^{\text {ét }}\right)$ is an étale $\mathbb{F}_{q}$-subshtuka of $\underline{M}_{q}(G)$. This yields a morphism $\underline{M}_{q}\left(G^{\text {ét }}\right) \rightarrow \underline{M}_{q}(G)_{\text {ét }}$. The equivalence of (i) shows that both morphisms are isomorphisms. This proves the compatibility of $\operatorname{Dr}_{q}$ and $\underline{M}_{q}$ with the canonical decompositions.

(iii) (b) By definition $G:=\operatorname{Dr}_{q}(\underline{M})$ is radicial over $S$ if $G(K) \rightarrow S(K)$ is injective for all fields $K$. This can be tested by applying the base change Spec $K \rightarrow S$. By (ii) and Propositions 4.2 and 2.7 the base change $G \times{ }_{S} \operatorname{Spec} K$ is connected if and only if $F_{M} \otimes \mathrm{id}_{K}$ is nilpotent. This implies (iii)(b) over $\operatorname{Spec} K$. It remains to show that $F_{M}$ is nilpotent locally on $S$ if $G$ is radicial. Locally on an affine open $\operatorname{Spec} R \subset S$ we may choose an $R$-basis of $M$ and write $F_{M}$ as an $r \times r$-matrix where $r=\operatorname{rk} M$. For every point $s \in S$, Proposition 2.7 implies that $F_{M}^{r}=0$ in $\kappa(s)^{r \times r}$. Therefore the entries of the matrix $F_{M}^{r}$ lie in the nil-radical of $R$. If $n$ is an integer such that their $q^{n}$-th powers are zero, then $F_{M}^{r(n+1)}=F_{M}^{r} \cdots \sigma_{q}^{n *}\left(F_{M}^{r}\right)=0$. This establishes (iii)(b).

(iii) (c) If locally on $S$ we choose an isomorphism $M \cong \bigoplus_{v=1}^{n} \mathcal{O}_{S} \cdot X_{v}$ and let $\left(t_{i j}\right)$ be the matrix of the morphism $F_{M}: \sigma_{q}^{*} M \rightarrow M$ with respect to the basis $\left(X_{1}, \ldots, X_{n}\right)$, then $\operatorname{Dr}_{q}(\underline{M})$ is the subscheme of $\mathbb{G}_{a, S}^{n}$, given by the system of equations

$$
X_{j}^{q}=\sum_{i=1}^{n} t_{i j} X_{i} \quad \text { for } j=1, \ldots, n .
$$

Therefore $\mathcal{O}_{\operatorname{Dr}_{q}(\underline{M})}$ is a free $\mathcal{O}_{S}$-module with basis $X_{1}^{m_{1}} \cdots X_{n}^{m_{n}}, 0 \leq m_{i}<q$. Thus $\operatorname{ord} \operatorname{Dr}_{q}(\underline{M}):=\operatorname{rk}_{\mathcal{O}_{s}} \mathcal{O}_{\operatorname{Dr}_{q}(\underline{M})}=q^{\mathrm{rk} M}$.

(iii) (d) In the presentation of $\ell_{\mathcal{D} r_{q}(\underline{M}) / S}$ given in (5.1) with

$$
I=\left(m^{\otimes q}-F_{M}\left(\sigma_{q}^{*} m\right): m \in M\right)
$$

and $I_{0}=\oplus_{n \geq 1} \operatorname{Sym}_{\mathcal{O}_{S}}^{n} M$, we use the isomorphisms of $\mathcal{O}_{S}$-modules $M \stackrel{\sim}{\rightarrow} I_{0} / I_{0}^{2}$, $m \mapsto m$ and $\sigma_{q}^{*} M \stackrel{\sim}{\rightarrow} I /\left(I I_{0}\right), b \sigma_{q}^{*} m=m \otimes b \mapsto b F_{M}\left(\sigma_{q}^{*} m\right)-b m^{\otimes q}$. Note that the latter is surjective by definition and injective because both $\sigma_{q}^{*} M$ and $I /\left(I I_{0}\right)$ are locally free $\mathcal{O}_{S}$-modules of the same rank. 
Remark 5.3 Finite locally free strict $\mathbb{F}_{q}$-module schemes over $S=\operatorname{Spec} R$ were equivalently described by Poguntke [Pogl7]. He defined the category $\mathbb{F}_{q}$-gr $r_{S}^{+, b}$ of finite locally free $\mathbb{F}_{q}$-module schemes $G=\operatorname{Spec} A$ that locally on $S$ can be embedded into $\mathbb{G}_{a, S}^{N}$ for some set $N$ and are balanced in the following sense. The $R$-module

$$
M_{p}(G):=\operatorname{Hom}_{S \text {-groups }}\left(G, \mathbb{G}_{a, S}\right)=\{x \in A: \Delta(x)=x \otimes 1+1 \otimes x\}
$$

of morphisms of group schemes over $R$ decomposes under the action of $\mathbb{F}_{q}$ on $G$ into eigenspaces

$$
M_{p}(G)_{p^{i}}:=\left\{m \in M_{p}(G):[\alpha](m)=\alpha^{p^{i}} \cdot m \text { for all } \alpha \in \mathbb{F}_{q}\right\},
$$

for $i \in \mathbb{Z} / e \mathbb{Z}$, where $q=p^{e}$. Now $G$ is balanced if the composition on the right with the relative $p$-Frobenius $F_{p, \mathbb{G}_{a, S}}$ of the additive group scheme $\mathbb{G}_{a, S}$ induces isomorphisms $M_{p}(G)_{p^{i}} \stackrel{\sim}{\rightarrow} M_{p}(G)_{p^{i+1}}$ for all $i=0, \ldots, e-2$. Note that it is neither required nor implied that $M_{p}(G)_{p^{e-1}} \rightarrow M_{p}(G)_{1}=: M_{q}(G)$ is also an isomorphism. The latter holds if and only if $G$ is étale by Theorem 5.2(ii).

Abrashkin [Abr06, 2.3.2] showed that every finite locally free strict $\mathbb{F}_{q}$-module scheme over $S$ belongs to $\mathbb{F}_{q}$-gr ${ }_{S}^{+, b}$. And Poguntke [Pog17, Theorem 1.4] conversely showed that $\operatorname{Dr}_{q}$ and $\underline{M}_{q}$ provide an anti-equivalence between the category of finite

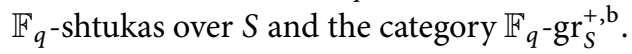

\section{Relation to Global Objects}

Without giving proofs, in this section we want to relate local shtukas and divisible local Anderson modules (defined in the next section), as well as finite $\mathbb{F}_{q}$-shtukas and finite locally free strict $\mathbb{F}_{q}$-module schemes to global objects like $A$-motives, global shtukas, Drinfeld modules, Anderson $A$-modules, etc. which are defined as follows. Let $C$ be a smooth, projective, geometrically irreducible curve over $\mathbb{F}_{q}$. For an $\mathbb{F}_{q}$-scheme $S$ we set $C_{S}:=C \times_{\mathbb{F}_{q}} S$ and we consider the endomorphism

$$
\sigma_{q}:=\operatorname{id}_{C} \otimes \operatorname{Frob}_{q, S}: C_{S} \longrightarrow C_{S} .
$$

Definition 6.1 (i) Let $n$ and $r$ be positive integers. A global shtuka of rank $r$ with $n$ legs over an $\mathbb{F}_{q}$-scheme $S$ is a tuple $\underline{\mathcal{N}}=\left(\mathcal{N}, c_{1}, \ldots, c_{n}, \tau_{\mathcal{N}}\right)$ consisting of

- a locally free sheaf $\mathcal{N}$ of rank $r$ on $C_{S}$,

- $\mathbb{F}_{q}$-morphisms $c_{i}: S \rightarrow C$ called the legs of $\underline{\mathcal{N}}$,

- an isomorphism $\tau_{\mathcal{N}}:\left.\left.\sigma_{q}^{*} \mathcal{N}\right|_{C_{s} \backslash \cup_{i} \Gamma_{c_{i}}} \stackrel{\sim}{\rightarrow} \mathcal{N}\right|_{C_{S} \backslash \cup_{i} \Gamma_{c_{i}}}$ outside the graphs $\Gamma_{c_{i}}$ of the $c_{i}$. In this article we will only consider the case where $\Gamma_{c_{i}} \cap \Gamma_{c_{j}}=\varnothing$ for $i \neq j$.

(ii) A global shtuka over $S$ is a Drinfeld shtuka if $n=2, \Gamma_{c_{1}} \cap \Gamma_{c_{2}}=\varnothing$, and $\tau_{\mathcal{N}}$ satisfies $\tau_{\mathcal{N}}\left(\sigma_{q}^{*} \mathcal{N}\right) \subset \mathcal{N}$ on $C_{S} \backslash \Gamma_{c_{2}}$ with cokernel locally free of rank 1 as $\mathcal{O}_{S}$-module, and $\tau_{\mathcal{N}}^{-1}(\mathcal{N}) \subset \sigma_{q}^{*} \mathcal{N}$ on $C_{S} \backslash \Gamma_{c_{1}}$ with cokernel locally free of rank 1 as $\mathcal{O}_{S}$-module.

Drinfeld shtukas were introduced by Drinfeld [Dri87] under the name $F$-sheaves.

An important class of special examples is defined as follows. Let $\infty \in C$ be a closed point and put $A:=\Gamma\left(C \backslash\{\infty\}, \mathcal{O}_{C}\right)$. Then $\operatorname{Spec} A=C \backslash\{\infty\}$. We will consider affine 
$A$-schemes $c: S=\operatorname{Spec} R \rightarrow \operatorname{Spec} A$ and the ideal

$$
J:=\left(a \otimes 1-1 \otimes c^{*}(a): a \in A\right) \subset A_{R}:=\underset{\mathbb{F}_{q}}{\otimes} R
$$

whose vanishing locus $\mathrm{V}(J)$ is the graph $\Gamma_{c}$ of the morphism $c$. The endomorphism $\sigma_{q}:=\mathrm{id}_{C} \otimes$ Frob $_{q, S}: C_{S} \rightarrow C_{S}$ induces the ring endomorphism

$$
\sigma_{q}^{*}:=\mathrm{id}_{A} \otimes \operatorname{Frob}_{q, R}: A_{R} \longrightarrow A_{R}, a \otimes b \longmapsto a \otimes b^{q}
$$

of $A_{R}$ for $a \in A$ and $b \in R$. The following definition generalizes Anderson's [And86] notion of $t$-motives, which is obtained as the special case, where $C=\mathbb{P}^{1}, A=\mathbb{F}_{q}[t]$ and $R$ is a field.

Definition 6.2 Let $d$ and $r$ be positive integers and let $S=\operatorname{Spec} R$ be an affine $A$-scheme. An effective A-motive of rank $r$ and dimension $d$ over $S$ is a pair $\underline{N}=$ $\left(N, \tau_{N}\right)$ consisting of a locally free $A_{R}$-module $N$ of rank $r$ and a morphism

$$
\tau_{N}: \sigma_{q}^{*} N \longrightarrow N
$$

of $A_{R}$-modules, such that coker $\tau_{N}$ is a locally free $R$-module of rank $d$ and $J^{d} \cdot$ coker $\tau_{N}=0$. More generally, an A-motive of rank $r$ over $S$ is a pair $\underline{N}=\left(N, \tau_{N}\right)$ consisting of a locally free $A_{R}$-module $N$ of rank $r$ and an isomorphism

$$
\tau_{N}:\left.\left.\sigma_{q}^{*} N\right|_{\operatorname{Spec} A_{R} \backslash \mathrm{V}(J)} \stackrel{\sim}{\longrightarrow} N\right|_{\operatorname{Spec} A_{R} \backslash \mathrm{V}(J)}
$$

outside the vanishing locus $\mathrm{V}(J)=\Gamma_{c}$ of $J$.

Example 6.3 (a) If $\underline{\mathcal{N}}=\left(\mathcal{N}, c_{1}, c_{2}, \tau_{\mathcal{N}}\right)$ is a global shtuka of rank $r$ over $S=$ Spec $R$ with two legs such that $c_{1}=c$ and $c_{2}: S \rightarrow\{\infty\} \subset C$, then

$$
\underline{N}(\underline{\mathcal{N}}):=\left(N, \tau_{N}\right):=\left(\Gamma\left(\operatorname{Spec} A_{R}, \mathcal{N}\right), \tau_{\mathcal{N}}\right)
$$

is an $A$-motive of rank $r$ over $S$.

(b) Conversely, if $\infty \in C\left(\mathbb{F}_{q}\right)$, every $A$-motive $\underline{N}=\left(N, \tau_{N}\right)$ over an affine $A$-scheme $c: S=\operatorname{Spec} R \rightarrow \operatorname{Spec} A$ can be obtained from a global shtuka

$$
\underline{\mathcal{N}}=\left(\mathcal{N}, c_{1}, c_{2}, \tau_{\mathcal{N}}\right)
$$

by taking $c_{1}=c$ and $c_{2}: S \rightarrow\{\infty\} \subset C$, and taking $\mathcal{N}$ as an extension to $C_{S}$ of the sheaf associated with $N$ on $\operatorname{Spec} A_{R}$, and $\tau_{\mathcal{N}}=\tau_{N}$.

These global objects give rise to finite and local shtukas, and that motivates the names for the latter.

Example 6.4 (a) Let $i: D \hookrightarrow C$ be a finite closed subscheme and let

$$
\underline{\mathcal{N}}=\left(\mathcal{N}, c_{1}, \ldots, c_{n}, \tau_{\mathcal{N}}\right)
$$

be a global shtuka of rank $r$ over $S$ such that $\tau_{\mathcal{N}}\left(\sigma_{q}^{*} \mathcal{N}\right) \subset \mathcal{N}$ in a neighborhood of $D_{S}:=D \times_{\mathbb{F}_{q}} S$. (For example, this is satisfied if $\underline{\mathcal{N}}$ is a Drinfeld-shtuka and $D_{S} \cap \Gamma_{c_{2}}=\varnothing$ or if $\underline{\mathcal{N}}$ is as in Example 6.3 with $\underline{N}(\underline{\mathcal{N}})$ an effective $A$-motive and $D \subset \operatorname{Spec} A$.) Then $\left(M, F_{M}\right):=\left(i^{*} \mathcal{N}, i^{*} \tau_{\mathcal{N}}\right)$ is a finite $\mathbb{F}_{q}$-shtuka over $S$, because $M$ is locally free over $S$ of rank $r \cdot \operatorname{dim}_{\mathbb{F}_{q}} \mathcal{O}_{D}$. The sense in which $\underline{\mathcal{N}}$ is global and $\left(M, F_{M}\right)$ is finite, is with 
respect to the coefficients; $\underline{\mathcal{N}}$ lives over all of $C$ and $\underline{M}$ lives over the finite scheme $D$. This example gave rise to the name finite $\mathbb{F}_{q}$-shtuka.

(b) Let $v \in C$ be a closed point defined by a sheaf of ideals $\mathfrak{p} \subset \mathcal{O}_{C}$, let $\hat{q}$ be the cardinality of the residue field $\mathbb{F}_{v}$ of $v$, let $f:=\left[\mathbb{F}_{v}: \mathbb{F}_{q}\right]$, and let $z \in \mathbb{F}_{q}(C)$ be a uniformizing parameter at $v$. Let $\underline{\mathcal{N}}=\left(\mathcal{N}, c_{1}, \ldots, c_{n}, \tau_{\mathcal{N}}\right)$ be a global shtuka of rank $r$ over $S=\operatorname{Spec} R$ such that for some $i$ the elements of $c_{i}^{*}(\mathfrak{p})$ are nilpotent in $R$. Set $\zeta:=$ $c_{i}^{*}(z) \in R$. Then the formal completion of $C_{S}$ along the graph $\Gamma_{c_{i}}$ of $c_{i}$ is canonically isomorphic to $\operatorname{Spf} R[[z]]$ by [AH14, Lemma 5.3]. The formal completion $M$ of $\left(\mathcal{N}, \tau_{\mathcal{N}}\right)$ along $\Gamma_{c_{i}}$ together with $\tau_{M}:=\tau_{\mathcal{N}}^{f}: \sigma_{q}^{f *} M\left[\frac{1}{z-\zeta}\right] \stackrel{\sim}{\rightarrow} M\left[\frac{1}{z-\zeta}\right]$ is a local shtuka over $S$ of rank $r$ (as in Definition 2.1 with $q$ and $\mathbb{F}_{q}\left[[z]\right.$ and $\sigma_{q}^{*}$ replaced by $\hat{q}$ and $\mathbb{F}_{v}[[z]$ and $\sigma_{q}^{f *}$ ). See [Harl7, §6] for more details. Again $\underline{M}$ is local with respect to the coefficients as it lives over the complete local ring $\left.\widehat{\mathcal{O}}_{C, v}=\mathbb{F}_{v} \llbracket z\right]$ of $C$ at $v$. This gave rise to the name local shtuka.

So far we have discussed the semi-linear algebra side given by shtukas. On the side of group schemes, an important source from which the corresponding strict $\mathbb{F}_{q^{-}}$ module schemes arise are Drinfeld $A$-modules, or more generally abelian Anderson $A$-modules. To define them, let $c: S=\operatorname{Spec} R \rightarrow \operatorname{Spec} A$ be an affine $A$-scheme. Recall that for a smooth commutative group scheme $E$ over $\operatorname{Spec} R$ the co-Lie module $\omega_{E}:=$ $\varepsilon_{E}^{*} \Omega_{E / R}^{1}$ is a locally free $R$-module of rank equal to the relative dimension of $E$ over $R$. Moreover, on the additive group scheme $\mathbb{G}_{a, R}=\operatorname{Spec} R[x]$ the elements $b \in R$, and in particular $c^{*}(a) \in R$ for $a \in \mathbb{F}_{q} \subset A$, act via endomorphisms $\psi_{b}: \mathbb{G}_{a, R} \rightarrow \mathbb{G}_{a, R}$ given by $\psi_{b}^{*}: R[x] \rightarrow R[x], x \mapsto b x$. This makes $\mathbb{G}_{a, R}$ into an $\mathbb{F}_{q}$-module scheme. In addition, let $\tau:=F_{q, \mathbb{G}_{a, R}}$ be the relative $q$-Frobenius endomorphism of $\mathbb{G}_{a, R}=$ Spec $R[x]$ given by $x \mapsto x^{q}$. It satisfies $\tau \circ \psi_{b}=\psi_{b q} \circ \tau$. We let

$$
R\{\tau\}:=\left\{\sum_{i=0}^{n} b_{i} \tau^{i}: n \in \mathbb{N}_{0}, b_{i} \in R\right\}
$$

with $\tau b=b^{q} \tau$ be the non-commutative polynomial ring in the variable $\tau$ over $R$. There is an isomorphism of rings $R\{\tau\} \stackrel{\sim}{\rightarrow} \operatorname{End}_{R \text {-groups, } \mathbb{F}_{q} \text {-lin }}\left(\mathbb{G}_{a, R}\right)$ sending an element $f=\sum_{i} b_{i} \tau^{i} \in R\{\tau\}$ to the $\mathbb{F}_{q}$-equivariant endomorphism $f: \mathbb{G}_{a, R} \rightarrow \mathbb{G}_{a, R}$ given by $f^{*}(x):=\sum_{i} b_{i} x^{q^{i}}$.

Definition 6.5 Let $d$ and $r$ be positive integers. An abelian Anderson A-module of rank $r$ and dimension $d$ over an affine $A$-scheme $c: \operatorname{Spec} R \rightarrow \operatorname{Spec} A$ is a pair $\underline{E}=(E, \varphi)$ consisting of a smooth affine group scheme $E$ over $\operatorname{Spec} R$ of relative dimension $d$ and a ring homomorphism $\varphi: A \rightarrow \operatorname{End}_{R \text {-groups }}(E), a \mapsto \varphi_{a}$ such that

(i) there is a faithfully flat ring homomorphism $R \rightarrow R^{\prime}$ for which $E \times_{R} \operatorname{Spec} R^{\prime} \cong$ $\mathbb{G}_{a, R^{\prime}}^{d}$ as $\mathbb{F}_{q}$-module schemes, where $\mathbb{F}_{q}$ acts on $E$ via $\varphi$ and $\mathbb{F}_{q} \subset A$;

(ii) $\left(a \otimes 1-1 \otimes c^{*} a\right)^{d} \cdot \omega_{E}=0$ for all $a \in A$ under the action of $a \otimes 1$ induced from $\varphi_{a}$ and the natural action of $1 \otimes b$ for $b \in R$ on the $R$-module $\omega_{E}$;

(iii) the set $N:=M_{q}(\underline{E}):=\operatorname{Hom}_{R \text {-groups, } \mathbb{F}_{q} \text {-lin }}\left(E, \mathbb{G}_{a, R}\right)$ of $\mathbb{F}_{q}$-equivariant homomorphisms of $R$-group schemes is a locally free $A_{R}$-module of rank $r$ under the 
action given on $m \in N$ by

$$
A \ni a: N \rightarrow N, m \mapsto m \circ \varphi_{a}, \quad R \ni b: N \rightarrow N, m \mapsto \psi_{b} \circ m .
$$

If $d=1$, this is called a Drinfeld A-module over $S$; $c f$. [Har17, Theorem 2.13].

The case in which $C=\mathbb{P}^{1}, A=\mathbb{F}_{q}[t]$, and $R$ is a field was considered by Anderson [And86] under the name abelian t-module. In [Har17, Theorem 2.10] we gave a proof of the following relative version of Anderson's theorem [And86, Theorem 1].

Theorem 6.6 If $\underline{E}=(E, \varphi)$ is an abelian Anderson A-module of rank $r$ and dimension $d$, we consider, in addition, on $N:=M_{q}(\underline{E})$, the map $\tau_{N}^{\mathrm{semi}}: m \mapsto F_{q, \mathbb{G}_{a, R}} \circ m$. Since $\tau_{N}^{\text {semi }}(b m)=b^{q} \tau_{N}^{\text {semi }}(m)$ for $b \in R$, the map $\tau_{N}^{\text {semi }}$ is $\sigma_{q}$-semilinear and induces an $A_{R}$-linear map $\tau_{N}: \sigma_{q}^{*} N \rightarrow N$ with $\tau_{N}^{\text {semi }}(m)=\tau_{N}\left(\sigma_{q}^{*} m\right)$. Then $\underline{M}_{q}(\underline{E}):=\left(N, \tau_{N}\right)$ is an effective A-motive of rank $r$ and dimension $d$. There is a canonical isomorphism of $R$-modules coker $\tau_{N} \stackrel{\sim}{\rightarrow} \omega_{E}, m \bmod \tau_{N}\left(\sigma_{q}^{*} N\right) \mapsto m^{*}(1)$, where $m^{*}(1)$ means the image of $1 \in \omega_{\mathbb{G}_{a, R}}=R$ under the induced R-homomorphism $m^{*}: \omega_{\mathbb{G}_{a, R}} \rightarrow \omega_{E}$.

The contravariant functor $\underline{E} \mapsto \underline{M}_{q}(\underline{E})$ is fully faithful. Its essential image consists of all effective A-motives $\underline{N}=\left(N, \tau_{N}\right)$ over $R$ for which there exists a faithfully flat ring homomorphism $R \rightarrow R^{\prime}$ such that $N \otimes_{R} R^{\prime}$ is a finite free left $R^{\prime}\{\tau\}$-module under the $\operatorname{map} \tau: N \rightarrow N, m \mapsto \tau_{N}\left(\sigma_{q}^{*} m\right)$.

Example 6.7 Let $\underline{E}=(E, \varphi)$ be an abelian Anderson $A$-module over an affine $A$-scheme $c$ : Spec $R \rightarrow \operatorname{Spec} A$, and let $\underline{N}:=\underline{M}_{q}(\underline{E})$ be its associated effective $A$ motive.

(a) Let $\mathfrak{a} \subset A$ be a non-zero ideal. By [Har17, Theorem 5.4] the a-torsion submodule of $E$, defined as the scheme-theoretic intersection $\underline{E}[\mathfrak{a}]:=\bigcap_{a \in \mathfrak{a}} \operatorname{ker}\left(\varphi_{a}: E \rightarrow E\right)$, is a finite locally free $A / \mathfrak{a}$-module scheme and a strict $\mathbb{F}_{q}$-module scheme over $S$, which satisfies $\underline{M}_{q}(\underline{E}[\mathfrak{a}])=\underline{N} / \mathfrak{a} \underline{N}$ and $\underline{E}[\mathfrak{a}]=\operatorname{Dr}_{q}(\underline{N} / \mathfrak{a} \underline{N})$.

(b) Let $\mathfrak{p} \subset A$ be a maximal ideal and assume that the elements of $c^{*}(\mathfrak{p}) \subset R$ are nilpotent. Let $\hat{q}$ be the cardinality of the residue field $\mathbb{F}_{\mathfrak{p}}:=A / \mathfrak{p}$ and let $f:=\left[\mathbb{F}_{\mathfrak{p}}: \mathbb{F}_{q}\right]$. We fix a uniformizing parameter $z \in \mathbb{F}_{q}(C)=\operatorname{Frac}(A)$ at $\mathfrak{p}$ and set $\zeta:=c^{*}(z) \in R$. We obtain an isomorphism $\mathbb{F}_{\mathfrak{p}}\left[[z] \stackrel{\sim}{\rightarrow} A_{\mathfrak{p}}:=\lim _{\longleftarrow} A / \mathfrak{p}^{n}\right.$. As in Example 6.4 the $J$-adic completion $M$ of $\underline{N}$ together with $\tau_{M}:=\tau_{N}^{f}: \overleftarrow{\sigma_{q}^{f *}} M \rightarrow M$ is an effective local shtuka $\underline{M}=\left(M, \tau_{M}\right)$ over $R$ of rank $r$ (as in Definition 2.1 with $q$ and $\mathbb{F}_{q}[[z]]$ and $\sigma_{q}^{*}$ replaced by $\hat{q}$ and $\mathbb{F}_{\mathfrak{p}}[[z]]$ and $\left.\sigma_{q}^{f *}\right)$. By [Har17, Theorem 6.6] the torsion module $\underline{E}\left[\mathfrak{p}^{n}\right]$ is a finite locally free strict $\mathbb{F}_{\mathfrak{p}}$-module scheme that satisfies $\operatorname{Dr}_{\hat{q}}\left(\underline{M} / \mathfrak{p}^{n} \underline{M}\right)=\underline{E}\left[\mathfrak{p}^{n}\right]$ and $\underline{M} / \mathfrak{p}^{n} \underline{M}=\underline{M} \hat{q}\left(\underline{E}\left[\mathfrak{p}^{n}\right]\right)$. Moreover, in the sense of Section 7 below, the inductive limit $\underline{E}\left[\mathfrak{p}^{\infty}\right]:=\lim \underline{E}\left[\mathfrak{p}^{n}\right]$ is a $\mathfrak{p}$-divisible local Anderson module over $R$ that satisfies $\operatorname{Dr}_{\hat{q}}(\underline{M})=\underline{E}\left[\overrightarrow{\mathfrak{p}^{\infty}}\right]$ and $\underline{M}=\underline{M}_{\hat{q}}\left(\underline{E}\left[\mathfrak{p}^{\infty}\right]\right)$ under the functors from Theorem 8.3. Note that condition (ii) of Definition 6.5 implies that $(z-\zeta)^{d}=0$ on $\omega_{\underline{E}\left[\mathfrak{p}^{n}\right]}$ for every $n$ and on $\omega_{\underline{E}\left[\mathfrak{p}^{\infty}\right]}:=\lim _{\longleftarrow} \omega_{\underline{E}\left[\mathfrak{p}^{n}\right]}$. 


\section{Divisible Local Anderson Modules}

The name divisible local Anderson module is motivated by Example 6.7 (b). These are the function field analogs of $p$-divisible groups. They were introduced in [Har09], but their definition in [Har09, \$3.1] and the claimed equivalence in [Har09, \$3.2] is false. We give the correct definition below analogously to Messing [Mes72, Chapter I, Definition 2.1]. We fix the following notation. For an fppf-sheaf of $\mathbb{F}_{q}[z]$-modules $G$ over a scheme $S$, we denote the kernel of $z^{n}: G \rightarrow G$ by $G\left[z^{n}\right]$. Clearly $\left(G\left[z^{n+m}\right]\right)\left[z^{n}\right]=$ $G\left[z^{n}\right]$ for all $n, m \in \mathbb{N}$.

Definition 7.1 A $z$-divisible local Anderson module over a scheme $S \in \mathcal{N i l p}_{\left.\mathbb{F}_{q} \llbracket \zeta\right\rceil}$ is a sheaf of $\mathbb{F}_{q}[[z]]$-modules $G$ on the big fppf-site of $S$ such that

(i) $G$ is $z$-torsion, that is, $G=\underset{n}{\lim } G\left[z^{n}\right]$.

(ii) $G$ is $z$-divisible, that is, $z: G \rightarrow G$ is an epimorphism.

(iii) For every $n$ the $\mathbb{F}_{q}$-module $G\left[z^{n}\right]$ is representable by a finite locally free strict $\mathbb{F}_{q}$-module scheme over $S$ (Definition 4.7).

(iv) Locally on $S$ there exists an integer $d \in \mathbb{Z}_{\geq 0}$, such that $(z-\zeta)^{d}=0$ on $\omega_{G}$ where $\omega_{G}:=\lim _{n} \omega_{G\left[z^{n}\right]}$.

We define the co-Lie module of a $z$-divisible local Anderson module $G$ over $S$ as $\omega_{G}:=$ $\lim _{G} \omega_{G\left[z^{n}\right]}$. We will see in Lemma 8.2 and Theorem 10.7 that $\omega_{G}$ is a finite locally free $\overleftarrow{\mathcal{O}_{S}}$-module and we define the dimension of $G$ as $\mathrm{rk} \omega_{G}$. It is locally constant on $S$.

A $z$-divisible local Anderson module is called (ind-)étale if $\omega_{G}=0$. Since $\omega_{G}$ surjects onto each $\omega_{G\left[z^{n}\right]}$ because $\omega_{i_{n}}: \omega_{G\left[z^{n+1}\right]} \rightarrow \omega_{G\left[z^{n}\right]}$ is an epimorphism, $\omega_{G}=0$ if and only if all $G\left[z^{n}\right]$ are étale; see Lemma 3.5.

A morphism of $z$-divisible local Anderson modules over $S$ is a morphism of fppf-sheaves of $\mathbb{F}_{q}[[z]]$-modules.

The category of $z$-divisible Anderson modules over $S$ is $\mathbb{F}_{q}[[z]]$-linear and an exact category in the sense of Quillen [Qui73, \$2].

Remark 7.2 We will frequently use that for a quasi-compact $S$-scheme $X$, any $S$-morphism $f: X \rightarrow \lim _{\longrightarrow} G\left[z^{n}\right]$ factors through $f: X \rightarrow G\left[z^{m}\right]$ for some $m$; see for example [HV11, Lemma 5.4].

Remark 7.3 (On axiom (iv) in Definition 7.1) Note the following difference to the theory of $p$-divisible groups. On a commutative group scheme, multiplication by $p$ always induces multiplication with the scalar $p$ on its co-Lie module. In the case of $\mathbb{F}_{q}[[z]$-module schemes, axiom (iv) is the appropriate substitute for this fact, taking into account Example 6.7. It allows that $z-\zeta$ is nilpotent on $\omega_{G\left[z^{n}\right]}$. Without axiom (iv) the $\mathcal{O}_{S}$-module $\omega_{G}$ is not necessarily finite; see Example 7.7.

Notation 7.4 Let $G$ be a $z$-divisible local Anderson module. We denote by $i_{n}$ the inclusion map $G\left[z^{n}\right] \hookrightarrow G\left[z^{n+1}\right]$ and by $i_{n, m}: G\left[z^{n}\right] \rightarrow G\left[z^{m+n}\right]$ the composite of the inclusions $i_{n+m-1} \circ \cdots \circ i_{n}$. We denote by $j_{n, m}$ the unique homomorphism $G\left[z^{m+n}\right] \rightarrow$ 
$G\left[z^{m}\right]$ that is induced by multiplication with $z^{n}$ on $G\left[z^{m+n}\right]$ such that $i_{m, n} \circ j_{n, m}=$ $z^{n} \operatorname{id}_{G\left[z^{m+n}\right]}$. Observe that also $j_{n, m} \circ i_{m, n}=z^{n} \operatorname{id}_{G\left[z^{m}\right]}$ for all $m, n \in \mathbb{N}$, as can be seen by composing with the $\mathbb{F}_{q}[z]$-equivariant monomorphism $i_{m, n}: G\left[z^{m}\right] \hookrightarrow G\left[z^{m+n}\right]$.

The following two propositions give an alternative characterization of divisible local Anderson modules, which is analogous to Tate's definition [Tat66] of $p$-divisible groups.

Proposition 7.5 Let $G$ be a $z$-divisible local Anderson module.

(i) For any $0 \leq m, n$ the following sequence of group schemes over $S$ is exact

$$
0 \rightarrow G\left[z^{n}\right] \stackrel{i_{n, m}}{\longrightarrow} G\left[z^{m+n}\right] \stackrel{j_{n, m}}{\longrightarrow} G\left[z^{m}\right] \rightarrow 0 .
$$

(ii) There is a locally constant function $h: S \rightarrow \mathbb{N}_{0}, s \mapsto h(s)$ such that the order of $G\left[z^{n}\right]$ equals $q^{n h}$. We call $h$ the height of the $z$-divisible local Anderson module $G$.

Proof (i) Since $z: G \rightarrow G$ is an epimorphism, $j_{n, m}$ is also. The rest of (i) is clear. Let $h:=\operatorname{rk}_{\mathcal{O}_{s}} \underline{M}_{q}(G[z])$. Then ord $G[z]=q^{h}$ by Theorem 5.2 (iii)(c). Now (ii) follows from (i) and the multiplicativity of the order.

Proposition 7.6 Let $\left(G_{n}, i_{n}: G_{n} \hookrightarrow G_{n+1}\right)_{n \in \mathbb{N}}$ be an inductive system of $\mathbb{F}_{q}[z]$-module schemes that are finite locally free strict $\mathbb{F}_{q}$-module schemes over $S$ satisfying the following conditions.

(i) $i_{n}$ induces an isomorphism $i_{n}: G_{n} \stackrel{\sim}{\rightarrow} G_{n+1}\left[z^{n}\right]$.

(ii) There is a locally constant function $h: S \rightarrow \mathbb{N}_{0}$ such that ord $G_{n}=q^{n h}$ for all $n$.

(iii) Locally on $S$ there exists an integer $d \in \mathbb{Z}_{\geq 0}$, such that $(z-\zeta)^{d}=0$ on $\omega_{G}$, where $\omega_{G}=\lim _{\longleftarrow} \omega_{G_{n}}$.

Then $G=\underset{\lim }{\longrightarrow} G_{n}$ is a $z$-divisible local Anderson module.

Proof From (i) it follows that $G_{n}=G_{m}\left[z^{n}\right] \subset G\left[z^{n}\right]$ for all $m \geq n$. Conversely let $x \in G\left[z^{n}\right](T)$ for an $S$-scheme $T$. On each quasi-compact open subscheme $U \subset T$ we can find an $m$ such that $\left.x\right|_{U} \in G_{m}(U)$ by Remark 7.2. Now $z^{n} x=0$ implies $\left.x\right|_{U} \in G_{m}\left[z^{n}\right](U)=G_{n}(U)$. In total, $x \in G_{n}(T)$. This shows that $G_{n}=G\left[z^{n}\right]$ and $G=\lim G\left[z^{n}\right]$ is $z$-torsion.

The quotient $G_{n} / G_{1}$ is a finite locally free group scheme over $S$ by Remark 3.1 (d). Its order is $q^{(n-1) h}$, by (ii) and the multiplicativity of the order. The natural map $z: G_{n} / G_{1} \hookrightarrow G_{n}\left[z^{n-1}\right] \cong G_{n-1}$ is a monomorphism and hence a closed immersion by Remark 3.1 (a). It must be an isomorphism because ord $\left(G_{n} / G_{1}\right)=\operatorname{ord}\left(G_{n-1}\right)$ by (ii). This proves that $z: G_{n} \rightarrow G_{n-1}$ is an epimorphism of fppf-sheaves. Let $x \in G(T)$ for an $S$-scheme $T$. Choose a quasi-compact open covering $\left\{U_{i}\right\}_{i}$ of $T$. For each $i$ we find by Remark 7.2 an integer $n_{i}$ such that $\left.x\right|_{U_{i}} \in G_{n_{i}}\left(U_{i}\right)$. By the above, there is a $y_{i} \in G_{n_{i}+1}\left(U_{i}\right) \subset G\left(U_{i}\right)$ with $z \cdot y_{i}=\left.x\right|_{U_{i}}$. This shows that $G$ is $z$-divisible. By (iii) it is a $z$-divisible local Anderson module.

Note that we require Definition 7.1(iv) and Proposition 7.6(iii) due to the following example that we do not want to consider a $z$-divisible local Anderson module. 
Example 7.7 Let $S$ be the spectrum of a ring $R$ in which $\zeta$ is zero, and let $G_{n}$ be the subgroup of $\mathbb{G}_{a, S}^{n}=\operatorname{Spec} R\left[x_{1}, \ldots, x_{n}\right]$ defined by the ideal $\left(x_{1}^{q}, \ldots, x_{n}^{q}\right)$. Make $G_{n}$ into an $\left.\mathbb{F}_{q} \llbracket z\right]$-module scheme by letting $z$ act through $z^{*}\left(x_{1}\right)=0$ and $z^{*}\left(x_{v}\right)=x_{v-1}$, for $1<v \leq n$. Define $i_{n}: G_{n} \rightarrow G_{n+1}$ as the inclusion of the closed subgroup scheme defined by the ideal $\left(x_{n+1}\right)$.

As in Proposition 7.6 one proves that $G:=\lim _{n} G_{n}$ satisfies axioms (i)-(iii) of Definition 7.1, but not (iv). Here $\omega_{G_{n}}=\bigoplus_{i=1}^{n} R \cdot \vec{d} x_{i} \cong R^{n}$, and so $\omega_{G}$ is not a finite $R$-module. Therefore we cannot drop the conditions (iv) in Definition 7.1 and (iii) in Proposition 7.6.

In the remainder of this section we introduce truncated $z$-divisible local Anderson modules.

Lemma 7.8 Let $n \in \mathbb{N}$ and let $G$ be an fppf-sheaf of $\mathbb{F}_{q}[z]$-modules over $S$, such that $G=G\left[z^{n}\right]$. Then the following conditions are equivalent.

(i) $G$ is a flat $\mathbb{F}_{q}[z] /\left(z^{n}\right)$-module.

(ii) $\operatorname{ker}\left(z^{n-i}\right)=\operatorname{im}\left(z^{i}\right)$ for $i=0, \ldots, n$, that is, the morphism $z^{i}: G \rightarrow G\left[z^{n-i}\right]$ is an epimorphism.

Proof (i) $\Rightarrow$ (ii) Because of (i), the multiplication with $z^{i}$ induces isomorphisms

$$
\mathbb{F}_{q}[z] /(z) \stackrel{\sim}{\longrightarrow} z^{i} \mathbb{F}_{q}[z] / z^{i+1} \mathbb{F}_{q}[z], \quad G / z G \stackrel{\sim}{\longrightarrow} z^{i} G / z^{i+1} G
$$

for $i \leq n-1$. This gives us $\operatorname{ker}\left(z^{n-1}\right) \subset \operatorname{im}(z)$, and the opposite inclusion $\operatorname{ker}\left(z^{n-1}\right) \supset$ $\operatorname{im}(z)$ follows from $G=G\left[z^{n}\right]$. Now $\operatorname{ker}\left(z^{n-i}\right) \subset \operatorname{ker}\left(z^{n-1}\right) \subset \operatorname{im}(z)$ implies that $\operatorname{ker}\left(z^{n-i}\right)=z \operatorname{ker}\left(z^{n-i+1}\right)=z \cdot z^{i-1} G=z^{i} G$, by induction on $i$.

(ii) $\Rightarrow$ (i) Taking $i=1$ implies $\operatorname{im}(z)=\operatorname{ker}\left(z^{n-1}\right)$, and hence multiplication with $z^{n-1}$ induces an isomorphism $G / z G \stackrel{\sim}{\rightarrow} z^{n-1} G$. Since this factors through the epimorphisms $G / z G \rightarrow z G / z^{2} G \rightarrow \cdots \rightarrow z^{n-1} G$, we see that each of these maps is an isomorphism. Thus we have $\operatorname{gr}^{\bullet}\left(\mathbb{F}_{q}[z] /\left(z^{n}\right)\right) \otimes_{\mathbb{F}_{q}} \operatorname{gr}^{0}(G) \stackrel{\sim}{\rightarrow} \operatorname{gr}^{\bullet}(G)$. Note that the ideal $(z) \subset \mathbb{F}_{q}[z] /\left(z^{n}\right)$ is nilpotent. Since $G / z G$ is flat over $\mathbb{F}_{q}[z] /(z)=\mathbb{F}_{q}$, [Bou61, Chapter III, \$5.2, Theorem 1] implies that $G$ is a flat $\mathbb{F}_{q}[z] /\left(z^{n}\right)$-module.

Definition 7.9 Let $d, n \in \mathbb{N}_{>0}$. A truncated $z$-divisible local Anderson module with order of nilpotence $d$ and level $n$ is an fppf-sheaf of $\mathbb{F}_{q}[z]$-modules over $S$, such that

(i) if $n \geq 2 d$, it is an $\mathbb{F}_{q}[z] /\left(z^{n}\right)$-module scheme $G$ that is finite locally free and strict as an $\mathbb{F}_{q}$-module scheme, such that $(z-\zeta)^{d}$ is homotopic to 0 on $\ell_{G / S}^{\bullet}$ and $G$ satisfies the equivalent conditions of Lemma 7.8;

(ii) if $n<2 d$, it is of the form $\operatorname{ker}\left(z^{n}: G \rightarrow G\right)$ for some truncated $z$-divisible local Anderson module $G$ with order of nilpotence $d$ and level $2 d$.

If $G$ is a $z$-divisible local Anderson module over $S \in \mathcal{N i l p}_{\mathbb{F}_{q} \llbracket \zeta \rrbracket}$ with $(z-\zeta)^{d}=0$ on $\omega_{G}$, we will see in Proposition 9.5 that $G\left[z^{n}\right]$ is a truncated $z$-divisible local Anderson module with order of nilpotence $d$ and level $n$. This justifies the name. 


\section{The Local Equivalence}

The category of $z$-divisible local Anderson modules over $S$ and the category of local shtukas over $S$ are both $\mathbb{F}_{q}[[z]$-linear. Our next aim is to extend Drinfeld's construction and the equivalence from Section 5 to an equivalence between the category of effective local shtukas over $S$ and the category of $z$-divisible local Anderson modules over $S$.

For every effective local shtuka $\underline{M}=\left(M, F_{M}\right)$ over $S$ we observe that

$$
\underline{M}=\lim \left(M / z^{n} M, F_{M} \bmod z^{n} M\right)
$$

and we set $\operatorname{Dr}_{q}(\underline{M}):=\underset{n}{\lim } \operatorname{Dr}_{q}\left(M / z^{n} M, F_{M} \bmod z^{n} M\right)$. The action of $\mathbb{F}_{q}[[z]$ on $M$ makes $\operatorname{Dr}_{q}(\underline{M})$ into an fppf-sheaf of $\mathbb{F}_{q}[[z]]$-modules on $S$. Conversely, for every $z$-divisible local Anderson module $G=\underset{\lim }{\longrightarrow} G\left[z^{n}\right]$ over $S$ we set

$$
\underline{M}_{q}(G)=\left(M_{q}(G), F_{M_{q}(G)}\right):={\underset{\lim }{\longleftarrow}}_{n}\left(M_{q}\left(G\left[z^{n}\right]\right), F_{M_{q}\left(G\left[z^{n}\right]\right)}\right) \text {. }
$$

Multiplication with $z$ on $G$ gives $M_{q}(G)$ the structure of an $\mathcal{O}_{S}[[z]]$-module.

Lemma 8.1 Let $G=\lim G\left[z^{n}\right]$ be a $z$-divisible local Anderson module of height $r$ over S; see Proposition 7.5. Then $M_{q}(G)$ is a locally free sheaf of $\mathcal{O}_{S} \llbracket[z]$-modules of rank $r$.

Proof Applying $\underline{M}_{q}$ to the exact sequence $0 \rightarrow G\left[z^{n}\right] \stackrel{i_{n}}{\rightarrow} G\left[z^{n+1}\right] \stackrel{z^{n}}{\rightarrow} G\left[z^{n+1}\right]$ yields an exact sequence of $\mathcal{O}_{s}[[z]]$-modules

$$
M_{q}\left(G\left[z^{n+1}\right]\right) \stackrel{z^{n}}{\longrightarrow} M_{q}\left(G\left[z^{n+1}\right]\right) \stackrel{M_{q}\left(i_{n}\right)}{\longrightarrow} M_{q}\left(G\left[z^{n}\right]\right) \longrightarrow 0 .
$$

We deduce from [Bou61, §III.2.11, Proposition 14 and Corollaire 1] that $M_{q}(G)$ is a finitely generated $\mathcal{O}_{S}[[z]]$-module and the canonical map $M_{q}(G) \rightarrow M_{q}\left(G\left[z^{n}\right]\right)$ identifies $M_{q}\left(G\left[z^{n}\right]\right)$ with $M_{q}(G) / z^{n} M_{q}(G)$.

We claim that multiplication with $z$ on $M_{q}(G)$ is injective. So let $\lim _{\longleftarrow}\left(f_{n}\right)_{n} \in$ $M_{q}(G), f_{n} \in M_{q}\left(G\left[z^{n}\right]\right)$ with $z \cdot f_{n}=0$ in $M_{q}\left(G\left[z^{n}\right]\right)$ for all $n$. To prove the claim consider the factorization

$$
z \cdot \operatorname{id}_{M_{q}\left(G\left[z^{n+1}\right]\right)}=M_{q}\left(j_{1, n}\right) \circ M_{q}\left(i_{n, 1}\right): M_{q}\left(G\left[z^{n+1}\right]\right) \longrightarrow M_{q}\left(G\left[z^{n+1}\right]\right)
$$

obtained from Notation 7.4. Theorem 5.2(ii) implies that $M_{q}\left(j_{1, n}\right)$ is injective, and hence $f_{n}=M_{q}\left(i_{n, 1}\right)\left(f_{n+1}\right)$ is zero for all $n$ as desired.

Locally on $\operatorname{Spec} R \subset S$ the $R$-module $M_{q}(G[z])$ is free. By Theorem 5.2 (iii)(c) its rank is $r$. Let $m_{1}, \ldots, m_{r}$ be representatives in $M_{q}(G)$ of an $R$-basis of $M_{q}(G[z])$ and consider the presentation

$$
0 \longrightarrow \operatorname{ker} \alpha \longrightarrow \bigoplus_{i=1}^{r} R\left[[z] m_{i} \stackrel{\alpha}{\longrightarrow} M_{q}(G) \longrightarrow 0\right.
$$

Note that $\alpha$ is surjective by Nakayama's Lemma [Eis95, Corollary 4.8] because $z$ is contained in the radical of $R[[z]$. The snake lemma applied to multiplication with $z$ 
on the sequence (8.1) yields the exact sequence

$$
0 \longrightarrow \operatorname{coker}(z: \operatorname{ker} \alpha \longrightarrow \operatorname{ker} \alpha) \longrightarrow \bigoplus_{i=1}^{r} R m_{i} \stackrel{\sim}{\longrightarrow} M_{q}(G[z]) \longrightarrow 0
$$

in which the right map is an isomorphism. This implies that multiplication with $z^{n}$ is surjective on $\operatorname{ker} \alpha$ for all $n$, and hence $\operatorname{ker} \alpha \subset \bigcap_{n} z^{n} \cdot\left(\oplus_{i=1}^{r} R\left[[z] m_{i}\right)=0\right.$ because $R[[z]]$ is $z$-adically separated. Therefore, $M_{q}(G)$ is locally on $S$ a free $\mathcal{O}_{S}[[z]$-module of rank $r$.

Recall from Theorem 5.2 (iii)(d) that the co-Lie complex $\ell_{G\left[z^{n}\right] / S}$ of $G\left[z^{n}\right]$ is canonically isomorphic to the complex of $\mathcal{O}_{S}$-modules

$$
0 \longrightarrow \sigma_{q}^{*} M_{q}\left(G\left[z^{n}\right]\right) \stackrel{F_{M_{q}\left(G\left[z^{n}\right]\right)}}{\longrightarrow} M_{q}\left(G\left[z^{n}\right]\right) \longrightarrow 0 .
$$

In particular, $n_{G\left[z^{n}\right]} \cong \operatorname{ker} F_{M_{q}\left(G\left[z^{n}\right]\right)}$ and $\omega_{G\left[z^{n}\right]} \cong \operatorname{coker} F_{M_{q}\left(G\left[z^{n}\right]\right)}$ for the $\mathcal{O}_{S}$-modules from Definition 3.4.

Lemma 8.2 Let $S \in \mathcal{N i l p}_{\mathbb{F}_{q} \llbracket \zeta \rrbracket}$ and let $G=\underset{\lim _{\longrightarrow}}{\longrightarrow}\left[z^{n}\right]$ be a $z$-divisible local Anderson module over $S$.

(i) Locally on $S$ there is an $N \in \mathbb{N}$ such that the morphism $i_{n}: G\left[z^{n}\right] \hookrightarrow G\left[z^{n+1}\right]$ induces an isomorphism $\omega_{G\left[z^{n+1}\right]} \stackrel{\sim}{\rightarrow} \omega_{G\left[z^{n}\right]}$ for all $n \geq N$.

(ii) The projective system $\left(n_{G\left[z^{n}\right]}\right)_{n}$ satisfies the Mittag-Leffler condition.

(iii) $\underline{M}_{q}(G)$ is an effective local shtuka over $S$ and coker $\left(F_{M_{q}(G)}\right)$ is canonically isomorphic to $\omega_{G}$. In particular, $\omega_{G}$ is a finite locally free $\mathcal{O}_{S}$-module.

Proof Working locally on $S$ we may assume that $\zeta^{N^{\prime}}=0$ in $\mathcal{O}_{S}$ and that $(z-\zeta)^{d} \omega_{G}=$ 0 for some integers $N^{\prime}$ and $d$. Let $N \geq \max \left\{N^{\prime}, d\right\}$ be an integer which is a power of $p$. Then $z^{N} \omega_{G}=\left(z^{N}-\zeta^{N}\right) \omega_{G}=(z-\zeta)^{N} \omega_{G}=0$.

(i) The closed immersion $i_{n}: G\left[z^{n}\right] \hookrightarrow G\left[z^{n+1}\right]$ induces an epimorphism

$$
\omega_{i_{n}}: \omega_{G\left[z^{n+1}\right]} \rightarrow \omega_{G\left[z^{n}\right]}
$$

and therefore $\omega_{G}$ surjects onto each $\omega_{G\left[z^{n}\right]}$. This implies that $z^{N} \omega_{G\left[z^{n}\right]}=0$ for all $n$. Applying Lemma 3.6 to the exact sequence (7.1) for $m=1$, and using $i_{1, n} \circ j_{n, 1}=$ $z^{n} \operatorname{id}_{G\left[z^{n+1}\right]}$, we obtain that $\operatorname{ker}\left(\omega_{G\left[z^{n+1}\right]} \rightarrow \omega_{G\left[z^{n}\right]}\right)=z^{n} \omega_{G\left[z^{n+1}\right]}$. Therefore

$$
\omega_{G\left[z^{n+1}\right]} \stackrel{\sim}{\longrightarrow} \omega_{G\left[z^{n}\right]}
$$

is an isomorphism for all $n \geq N$.

To prove (ii) we fix an $n \geq N$. We abbreviate the $\mathcal{O}_{s}$-modules $M_{q}\left(G\left[z^{k}\right]\right)$ by $M_{k}$ and the map $F_{M_{q}\left(G\left[z^{k}\right]\right)}$ by $F_{k}$. From Proposition 7.5 and Theorem 5.2(ii) we have an exact sequence

$$
0 \rightarrow M_{k} \stackrel{M_{q}\left(j_{n, k}\right)}{\longrightarrow} M_{n+k} \stackrel{M_{q}\left(i_{n, k}\right)}{\longrightarrow} M_{n} \rightarrow 0
$$


It remains exact after applying $\sigma_{q}^{*}$ because $M_{n}$ is locally free. For all $k$, we consider the commutative diagrams,

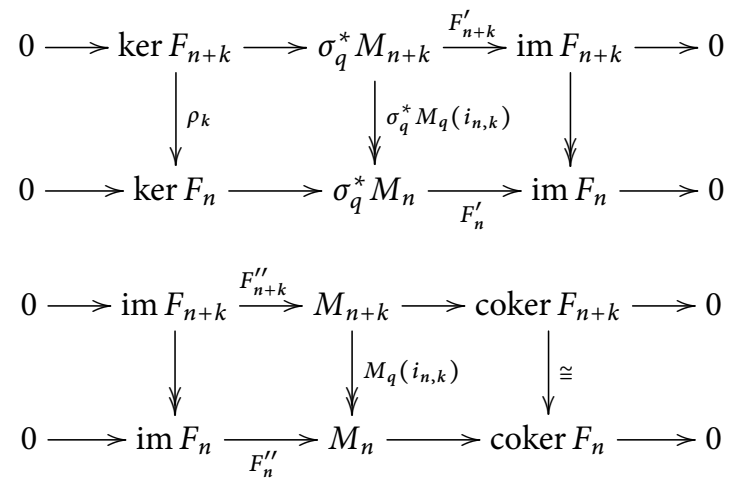

where we have split $F_{n}=F_{n}^{\prime \prime} \circ F_{n}^{\prime}$ with $F_{n}^{\prime}$ surjective and $F_{n}^{\prime \prime}$ injective, and where the vertical map on the right in the second diagram is an isomorphism by the identification coker $F_{n}=\omega_{G\left[z^{n}\right]}$ from Theorem 5.2 (iii) (d) and by what we proved in (i) above. We denote the vertical map on the left in the first diagram by $\rho_{k}$. The snake lemma applied to both diagrams yields the following exact sequence

$$
\sigma_{q}^{*} M_{k} \stackrel{F_{k}}{\rightarrow} M_{k} \longrightarrow \operatorname{coker} \rho_{k} \longrightarrow 0 .
$$

Therefore coker $\rho_{k} \cong \operatorname{coker} F_{k}=\omega_{G\left[z^{k}\right]}$. In the diagram

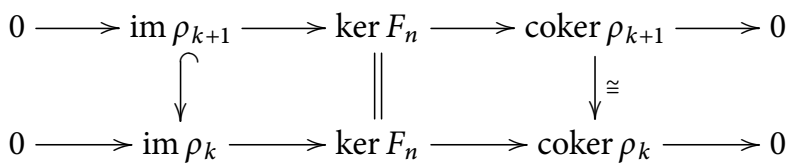

the vertical map on the right is an isomorphism for $k \geq N$ by what we have proved in (i) above. Therefore the image of $\rho_{k}$ stabilizes for $k \geq N$; that is, $n_{G\left[z^{n}\right]}=\operatorname{ker} F_{n}$ satisfies the Mittag-Leffler condition. Note that $\left(\operatorname{im} F_{n}\right)_{n}$ also satisfies the MittagLeffler condition. We will use this for proving (iii).

(iii) We still abbreviate $M_{q}\left(G\left[z^{n}\right]\right)$ by $M_{n}$ and $F_{M_{q}\left(G\left[z^{n}\right]\right)}$ by $F_{n}$. The maps

$$
F_{n}: \sigma_{q}^{*} M_{n} \rightarrow M_{n}
$$

give us two short exact sequences of projective systems

$$
0 \longrightarrow \operatorname{ker} F_{n} \longrightarrow \sigma_{q}^{*} M_{n} \longrightarrow \operatorname{im} F_{n} \longrightarrow 0
$$

and

$$
0 \longrightarrow \operatorname{im} F_{n} \longrightarrow M_{n} \longrightarrow \text { coker } F_{n} \longrightarrow 0 .
$$

Taking the projective limit, using the Mittag-Leffler conditions via [Har77, Proposition II.9.1(b)], the isomorphism $\sigma_{q}^{*}\left(M_{q}(G)\right) \cong \lim \sigma_{q}^{*}\left(M_{n}\right)$ (which is due to the flatness of $M_{q}(G)$ over $\left.\mathcal{O}_{S}\right)$, and combining both exact sequences we obtain an exact sequence

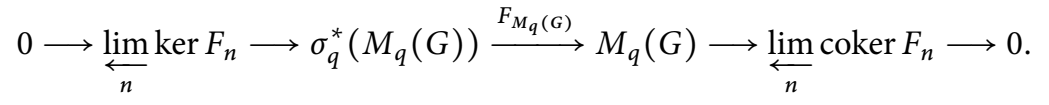


This shows that $\omega_{G}:=\lim \omega_{G\left[z^{n}\right]}=\lim \operatorname{coker} F_{n}=\operatorname{coker} F_{M_{q}(G)}$, which is finite locally free over $\mathcal{O}_{S}$ by Lemma 2.3. Furthermore, condition (iv) of Definition 7.1 implies that $(z-\zeta)^{d}$ annihilates coker $F_{M_{q}(G)}$. This proves that the map

$$
F_{M_{q}(G)}: \sigma_{q}^{*}\left(M_{q}(G)\right)\left[\frac{1}{z-\zeta}\right] \longrightarrow M_{q}(G)\left[\frac{1}{z-\zeta}\right]
$$

is surjective. As both modules are locally free over $\mathcal{O}_{S}\left[[z]\left[\frac{1}{z-\zeta}\right]\right.$ of the same rank, the map is an isomorphism. Thus $\underline{M}_{q}(G)$ is an effective local shtuka.

We can now prove the following theorem. It generalizes [And93, \$3.4], which treated the case of formal $\mathbb{F}_{q} \llbracket[z]$-modules and which we state in (i).

\section{Theorem 8.3 Let $S \in \mathcal{N i l p}_{\mathbb{F}_{q} \llbracket \zeta \rrbracket}$.}

(i) The two contravariant functors $\operatorname{Dr}_{q}$ and $\underline{M}_{q}$ are mutually quasi-inverse antiequivalences between the category of effective local shtukas over $S$ and the category of $z$-divisible local Anderson modules over S.

(ii) Both functors are $\mathbb{F}_{q}[[z]]$-linear, map short exact sequences to short exact sequences, and preserve (ind-)étale objects.

Suppose furthermore that $\underline{M}=\left(M, F_{M}\right)$ is an effective local shtuka over $S$ and let $G=\operatorname{Dr}_{q}(\underline{M})$ be its associated $z$-divisible local Anderson module. Then the following hold.

(i) $G$ is a formal $\mathbb{F}_{q}\left[[z]\right.$-module (Definition 1.1) if and only if $F_{M}$ is topologically nilpotent.

(ii) The height (see Proposition 7.5) and dimension of $G$ are equal to the rank and dimension of $\underline{M}$.

(iii) The $\mathcal{O}_{S}[[z]]$-modules $\omega_{\operatorname{Dr}_{q}(\underline{M})}$ and coker $F_{M}$ are canonically isomorphic.

Proof (i) We already saw in Lemma 8.2(iii) that $\underline{M}_{q}$ sends $z$-divisible local Anderson modules to effectve local shtukas. To prove the converse we use Proposition 7.6. Let $\underline{M}=\left(M, F_{M}\right)$ be an effective local shtuka over $S$ and abbreviate $\underline{M} / z^{n} \underline{M}=: \underline{M}_{n}=$ $\left(M_{n}, F_{M_{n}}\right)$ and $G_{n}:=\operatorname{Dr}_{q}\left(\underline{M}_{n}\right)$. Then $G:=\operatorname{Dr}_{q}(\underline{M})=\underline{\lim } G_{n}$. Consider the locally constant function $h:=\operatorname{rk}_{\mathcal{O}_{s} \llbracket z \rrbracket} M$ on $S$. It satisfies $\operatorname{rk}_{\mathcal{O}_{S}}\left(\vec{M}_{n}\right)=n h$. By Theorem 5.2 the $G_{n}$ are finite locally free strict $\mathbb{F}_{q}$-module schemes over $S$ of order $q^{n h}$, and the exact sequence of finite $\mathbb{F}_{q}$-shtukas

$$
\underline{M}_{n+1} \stackrel{z^{n}}{\longrightarrow} \underline{M}_{n+1} \longrightarrow \underline{M}_{n} \longrightarrow 0
$$

yields an exact sequence of group schemes $0 \longrightarrow G_{n} \longrightarrow G_{n+1} \stackrel{z^{n}}{\longrightarrow} G_{n+1}$. This implies that $G_{n}=\operatorname{ker}\left(z^{n}: G_{n+1} \rightarrow G_{n+1}\right)=: G_{n+1}\left[z^{n}\right]$. By Lemma 2.3 we know that locally on $S$ there exist positive integers $e^{\prime}, N$ such that $(z-\zeta)^{e^{\prime}}=0$ on coker $F_{M}$ and $z^{N}=0$ on 
coker $F_{M}$. Applying the snake lemma to the diagram

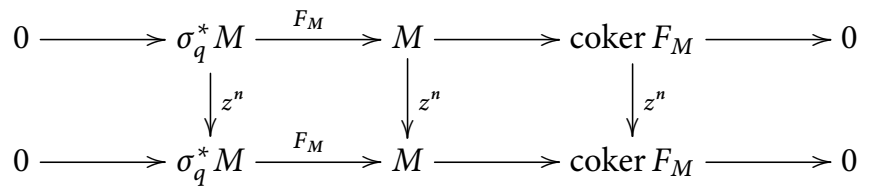

shows that coker $F_{M} \rightarrow \operatorname{coker} F_{M_{n}}$ is an isomorphism for $n \geq N$. Therefore by Theorem 5.2 (iii)(d) $\omega_{G}:=\lim \omega_{G_{n}}=\lim \operatorname{coker}\left(F_{M_{n}}\right)=\operatorname{coker} F_{M}$. This establishes (iii) and implies $(z-\zeta)^{e^{\prime}}=0$ on $\omega_{G}$. Therefore $G=\lim _{\longrightarrow} G_{n}$ is a $z$-divisible local Anderson module by Proposition 7.6. By Theorem 5.2 the functors $\operatorname{Dr}_{q}$ and $\underline{M}_{q}$ are quasi-inverse to each other. This proves (i).

(ii) From our proof above, the height of $\operatorname{Dr}_{q}(\underline{M})$ equals the rank of $\underline{M}$. The equality of dimensions follows from (iii).

(ii) The $\left.\mathbb{F}_{q}[z]\right]$-linearity of the functors is clear by construction. From (iii) it follows that both functors $\operatorname{Dr}_{q}$ and $\underline{M}_{q}$ preserve (ind-)étale objects. To prove the exactness of $\operatorname{Dr}_{q}$ let $0 \rightarrow \underline{M}^{\prime \prime} \rightarrow \underline{M} \rightarrow \underline{M}^{\prime} \rightarrow 0$ be a short exact sequence of effective local shtukas. Modulo $z^{n}$, it yields a short exact sequence of finite $\mathbb{F}_{q}$-shtukas $0 \rightarrow \underline{M}_{n}^{\prime \prime} \rightarrow \underline{M}_{n} \rightarrow \underline{M}_{n}^{\prime} \rightarrow 0$, where $\underline{M}_{n}^{\prime \prime}:=\underline{M}^{\prime \prime} / z^{n} \underline{M}^{\prime \prime}$, etc. Theorem 5.2 produces the exact sequence $0 \rightarrow G^{\prime}\left[z^{n}\right] \rightarrow G\left[z^{n}\right] \rightarrow G^{\prime \prime}\left[z^{n}\right] \rightarrow 0$, where $G=\operatorname{Dr}_{q}(\underline{M})$, $G^{\prime}=\operatorname{Dr}_{q}\left(\underline{M}^{\prime}\right), G^{\prime \prime}=\operatorname{Dr}_{q}\left(\underline{M}^{\prime \prime}\right)$. This implies that $0 \rightarrow G^{\prime} \rightarrow G \rightarrow G^{\prime \prime} \rightarrow 0$ is exact, because taking direct limits in the category of sheaves is an exact functor.

Conversely, let $0 \rightarrow G^{\prime} \rightarrow G \rightarrow G^{\prime \prime} \rightarrow 0$ be a short exact sequence of $z$-divisible local Anderson modules. Since multiplication with $z^{n}$ is an epimorphism on $G^{\prime \prime}$, the snake lemma yields the exact sequence of finite locally free strict $\mathbb{F}_{q}$-module schemes $0 \rightarrow G^{\prime}\left[z^{n}\right] \rightarrow G\left[z^{n}\right] \rightarrow G^{\prime \prime}\left[z^{n}\right] \rightarrow 0$. Theorem 5.2 implies that the sequence $0 \rightarrow \underline{M}_{n}^{\prime \prime} \rightarrow \underline{M}_{n} \rightarrow \underline{M}_{n}^{\prime} \rightarrow 0$ is exact, where $\underline{M}=\underline{M}_{q}(G), \underline{M}^{\prime}=\underline{M}_{q}\left(G^{\prime}\right), \underline{M}^{\prime \prime}=$ $\underline{M}_{q}\left(G^{\prime \prime}\right)$. Since $\left\{\underline{M}_{n}^{\prime \prime}\right\}$ satisfies the Mittag-Leffler condition, we obtain the exactness of $0 \rightarrow \underline{M}^{\prime \prime} \rightarrow \underline{M} \rightarrow \underline{M}^{\prime} \rightarrow 0$.

(i) Let $G=\operatorname{Dr}_{q}(\underline{M})$. In Proposition 10.11 we will see that $G$ is a formal $\mathbb{F}_{q}[[z]$-module if and only if $G[z]=\operatorname{ker}(z: G \rightarrow G)$ is radicial, which by Theorem 5.2 (iii)(b) is equivalent to $F_{M_{1}}=\left(F_{M} \bmod z\right)$ being nilpotent locally on $S$. The latter is the case if and only if locally on $S$ there is an integer $n$ such that $\left(F_{M}\right)^{n} \equiv 0 \bmod z$, that is, if and only if $F_{M}$ is topologically nilpotent.

Corollary 8.4 Let $S \in \mathcal{N i l p}_{\mathbb{F}_{q}[[\zeta]]}$ and let $f: G^{\prime} \rightarrow$ G be a monomorphism of $z$-divisible local Anderson modules over $S$. Then the quotient sheaf $G / G^{\prime}$ is a $z$-divisible local Anderson module over $S$.

Proof Since the question is local on $S$, we can assume that $S=\operatorname{Spec} R$ is affine. For all $n$, the induced map $G^{\prime}\left[z^{n}\right] \rightarrow G\left[z^{n}\right]$ is a monomorphism, hence a closed immersion by Remark 3.1 (a). By Lemma 4.6 it is strict $\mathbb{F}_{q}$-linear in the sense of Faltings [Fal02, Definition 1], and by [Fal02, Proposition 2] the cokernel $G_{n}^{\prime \prime}:=G\left[z^{n}\right] / G^{\prime}\left[z^{n}\right]$ is a strict $\mathbb{F}_{q}$-module scheme that is finite locally free by Remark 3.1 (d). By Theorem 5.2 
this induces the exact sequence of finite $\mathbb{F}_{q}$-shtukas

$$
0 \rightarrow \underline{M}_{q}\left(G_{n}^{\prime \prime}\right) \rightarrow \underline{M}_{q}\left(G\left[z^{n}\right]\right) \rightarrow \underline{M}_{q}\left(G^{\prime}\left[z^{n}\right]\right) \rightarrow 0 .
$$

In the diagram

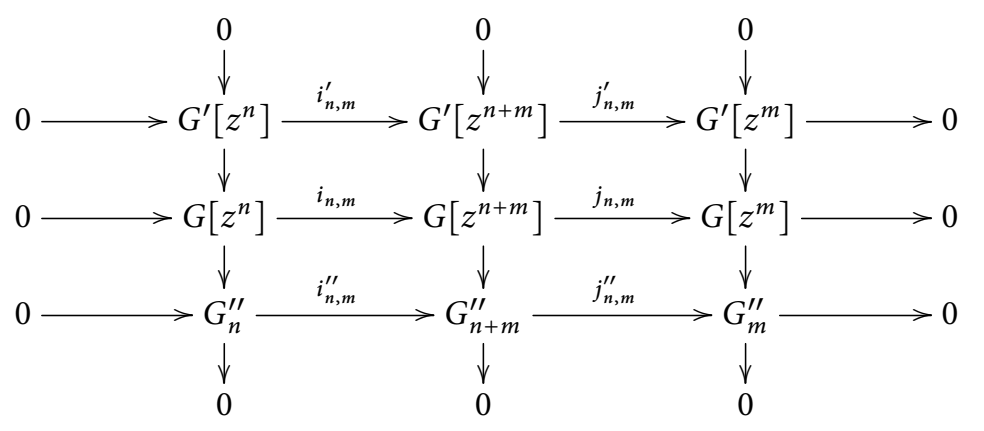

the columns are exact by definition of $G_{n}^{\prime \prime}$ and the two upper rows are exact by Proposition 7.5. By the snake lemma, this defines the exact sequence in the bottom row. By Theorem 5.2, this implies that $\underline{M}_{q}\left(i_{n, 1}^{\prime \prime}\right): \underline{M}_{q}\left(G_{n+1}^{\prime \prime}\right) \rightarrow \underline{M}_{q}\left(G_{n}^{\prime \prime}\right)$ is surjective for all $n$. In particular, the projective system $\underline{M}_{q}\left(G_{n}^{\prime \prime}\right)$ satisfies the Mittag-Leffler condition, and the morphism $\underline{M}_{q}(f): \underline{M}:=\underline{M}_{q}(G) \rightarrow \underline{M}^{\prime}:=\underline{M}_{q}\left(G^{\prime}\right)$ of effective local shtukas corresponding to $f$ by Theorem 8.3 is surjective by [Har77, Proposition II.9.1(b)]. The kernel $\underline{M}^{\prime \prime}:=\operatorname{ker} \underline{M}_{q}(f)=\lim \underline{M}_{q}\left(G_{n}^{\prime \prime}\right)$ is a locally free $R[[z]]$-module with a morphism $F_{M^{\prime \prime}}: \sigma_{q}^{*} M^{\prime \prime} \rightarrow M^{\prime \prime}$ inducing an isomorphism $F_{M^{\prime \prime}}: \sigma_{q}^{*} M^{\prime \prime}\left[\frac{1}{z-\zeta}\right] \rightarrow M^{\prime \prime}\left[\frac{1}{z-\zeta}\right]$, because this is true for $\underline{M}$ and $\underline{M}^{\prime}$. Thus $\underline{M}^{\prime \prime}$ is an effective local shtuka over $S$. Applying the snake lemma to the (injective) multiplication with $z^{n}$ on the sequence $0 \rightarrow \underline{M}^{\prime \prime} \rightarrow \underline{M} \rightarrow \underline{M}^{\prime} \rightarrow 0$ shows that $\underline{M}^{\prime \prime} / z^{n} \underline{M}^{\prime \prime}=\underline{M}_{q}\left(G_{n}^{\prime \prime}\right)$. Therefore, Theorem 8.3 implies that $G / G^{\prime}=\operatorname{Dr}_{q}\left(\underline{M}^{\prime \prime}\right)=\underline{\lim }_{\longrightarrow} G_{n}^{\prime \prime}$ is a $z$-divisible local Anderson module over $S$.

\section{Frobenius, Verschiebung and Deformations of Local Shtukas}

Definition 9.1 Let $G$ be an fppf-sheaf of groups over an $\mathbb{F}_{q}$-scheme $S$. For $n \in \mathbb{N}_{0}$ we let $G\left[F_{q}^{n}\right]$ be the kernel of the relative $q^{n}$-Frobenius $F_{q^{n}, G}: G \rightarrow \sigma_{q^{n}}^{*} G$ of $G$ over $S$.

Let $S \in \mathcal{N} \operatorname{Nilp}_{\mathbb{F}_{q}[[\zeta]]}$. Later we will assume that $\zeta=0$ in $\mathcal{O}_{S}$. Let $G$ be a $z$-divisible local Anderson module over $S$ and let $\underline{M}=\left(M, F_{M}\right)=\underline{M}_{q}(G)$ be its associated local shtuka from Theorem 8.3. Then the $q$-Frobenius morphism $F_{q, G}:=\lim _{\longrightarrow} F_{q, G\left[z^{n}\right]}: G \rightarrow$ $\sigma_{q}^{*} G$ corresponds by diagram (5.2) to the morphism

$\underline{M}_{q}\left(F_{q, G}\right)=F_{M}: M_{q}\left(\sigma_{q}^{*} G\right)=\sigma_{q}^{*} M_{q}(G) \longrightarrow M_{q}(G), \quad m \longmapsto m \circ F_{q, G}=F_{M}(m)$.

In addition to the $q$-Frobenius, $G$ carries a $q$-Verschiebung that is identically zero by Theorem 5.1. Therefore, if $\zeta=0$ in $\mathcal{O}_{S}$, we will introduce a " $z$-Verschiebung" in Remark 9.3 and Corollary 9.4, which is more useful for $z$-divisible local Anderson modules. We begin with the following lemma. 
Lemma 9.2 Let $M$ be an effective local shtuka with $(z-\zeta)^{d}=0$ on coker $F_{M}$. Then there exists a uniquely determined homomorphism of $\mathcal{O}_{S}[[z]]$-modules $V_{M}: M \rightarrow \sigma_{q}^{*} M$ with $F_{M} \circ V_{M}=(z-\zeta)^{d} \cdot \operatorname{id}_{M}$ and $V_{M} \circ F_{M}=(z-\zeta)^{d} \cdot \operatorname{id}_{\sigma_{q}^{*} M}$.

Proof Since $F_{M}$ is injective by Lemma 2.3 and $(z-\zeta)^{d}=0$ on coker $F_{M}$, the lemma follows from diagram (9.1).

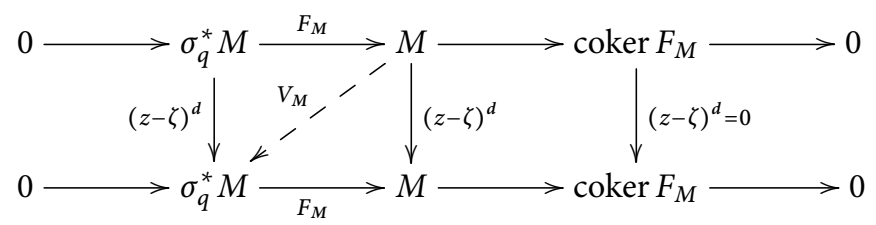

Remark 9.3 If $\zeta=0$ in $\mathcal{O}_{S}$, the Frobenius $f:=F_{M}: \sigma_{q}^{*} \underline{M}\left[\frac{1}{z}\right] \stackrel{\sim}{\rightarrow} \underline{M}\left[\frac{1}{z}\right]$ satisfies $F_{M} \circ \sigma_{q}^{*} f=F_{M} \circ \sigma_{q}^{*} F_{M}=f \circ \sigma_{q}^{*} F_{M}$, and hence is a quasi-isogeny between the local shtukas $\sigma_{q}^{*} \underline{M}=\left(\sigma_{q}^{*} M, \sigma_{q}^{*} F_{M}\right)$ and $\underline{M}$. Likewise, if $\underline{M}$ is effective with $(z-\zeta)^{d}=0$ on coker $F_{M}$, the homomorphism $V_{M}$ from Lemma 9.2 is an isogeny $V_{z^{d}, \underline{M}}:=V_{M}: \underline{M}\left[\frac{1}{z}\right] \stackrel{\sim}{\rightarrow} \sigma_{q}^{*} \underline{M}\left[\frac{1}{z}\right]$, called the $z^{d}$-Verschiebung of $\underline{M}$. It satisfies $F_{M} \circ$ $V_{z^{d}, \underline{M}}=z^{d} \cdot \operatorname{id}_{\underline{M}}$ and $V_{z^{d}, \underline{M}} \circ F_{M}=z^{d} \cdot \operatorname{id}_{\sigma_{q}^{*} \underline{M}}$. Indeed, $\zeta=0=\zeta^{q}$ implies that the following diagram is commutative

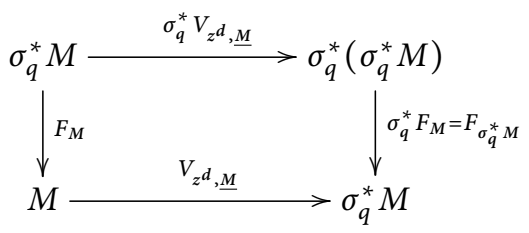

as

$$
\begin{aligned}
F_{\sigma_{q}^{*} M} \circ \sigma_{q}^{*} V_{z^{d}, \underline{M}} & =\sigma_{q}^{*} F_{M} \circ \sigma_{q}^{*} V_{z^{d}, \underline{M}}=\sigma_{q}^{*}\left((z-\zeta)^{d} \cdot \operatorname{id}_{M}\right) \\
& =\left(z-\zeta^{q}\right)^{d} \cdot \operatorname{id}_{\sigma_{q}^{*} M}=V_{z^{d}, \underline{M}} \circ F_{M} .
\end{aligned}
$$

Corollary 9.4 Assume that $\zeta=0$ in $\mathcal{O}_{s}$. Let $G$ be a $z$-divisible local Anderson module over $S$ with $(z-\zeta)^{d}=0$ on $\omega_{G}$. Then there is a uniquely determined morphism $V_{z^{d}, G}: \sigma_{q}^{*} G \rightarrow G$ with $F_{q, G} \circ V_{z^{d}, G}=z^{d} \cdot \operatorname{id}_{\sigma_{q}^{*} G}$ and $V_{z^{d}, G} \circ F_{q, G}=z^{d} \cdot \operatorname{id}_{G}$. It is called the $z^{d}$-Verschiebung of $G$. In particular, $G\left[F_{q}^{n}\right]$ is contained in $G\left[z^{\text {nd }}\right]$ and $\operatorname{ker}\left(V_{z^{d}, G}^{n}: \sigma_{q^{n}}^{*} G \rightarrow G\right) \subset \sigma_{q^{n}}^{*} G\left[z^{n d}\right]$ for all $n$.

Proof Let $\underline{M}=\underline{M}_{q}(G)$ be the effective local shtuka associated with $G$. Since

$$
(z-\zeta)^{d}=0
$$

on $\omega_{G}=\operatorname{coker} F_{M}$, the $z^{d}$-Verschiebung $V_{z^{d}, M}$ of $\underline{M}$ from Remark 9.3 corresponds by Theorem 8.3 to a morphism $V_{z^{d}, G}:=\operatorname{Dr}_{q}\left(V_{z^{d}, \underline{M}}\right): \sigma_{q}^{*} G \rightarrow G$ with $F_{q, G} \circ V_{z^{d}, G}=$ 
$z^{d} \cdot \operatorname{id}_{\sigma_{q}^{*} G}$ and $V_{z^{d}, G} \circ F_{q, G}=z^{d} \cdot \operatorname{id}_{G}$, and hence $V_{z^{d}, G}^{n} \circ F_{q, G}^{n}=z^{n d} \cdot \operatorname{id}_{G}$ and $F_{q, G}^{n} \circ$ $V_{z^{d}, G}^{n}=z^{n d} \cdot \operatorname{id}_{\sigma_{q^{n}}^{*} G}$. This proves the corollary.

Proposition 9.5 Let $G$ be a $z$-divisible local Anderson module with $(z-\zeta)^{d}=0$ on $\omega_{G}$, and let $n \in \mathbb{N}$. Then $G\left[z^{n}\right]:=\operatorname{ker}\left(z^{n}: G \rightarrow G\right)$ is a truncated $z$-divisible local Anderson module with order of nilpotence $d$ and level $n$; see Definition 7.9.

Proof The equivalent conditions of Lemma 7.8 for the $\mathbb{F}_{q}[z] /\left(z^{n}\right)$-module scheme $G\left[z^{n}\right]$ follow from Proposition 7.5 by considering, for all $v=0, \ldots, n$, the commutative diagram

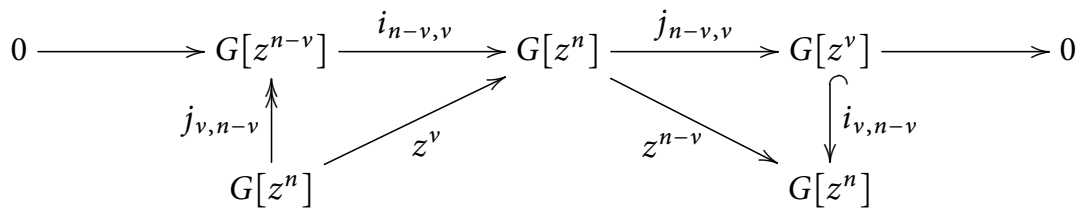

in which $i_{v, n-v}$ is a monomorphism and $j_{v, n-v}$ an epimorphism, and hence

$$
\operatorname{ker}\left(z^{n-v}\right)=\operatorname{ker}\left(j_{n-v, v}\right)=\operatorname{im}\left(i_{n-v, v}\right)=\operatorname{im}\left(z^{v}\right) .
$$

By Theorem 8.3(iii), $(z-\zeta)^{d}=0$ on $\operatorname{coker} F_{M_{q}(G)}$. We reduce the map $V_{M_{q}(G)}$ from Lemma 9.2 modulo $z^{n}$ to obtain a homomorphism

$$
V_{M_{q}\left(G\left[z^{n}\right]\right)}: M_{q}\left(G\left[z^{n}\right]\right) \rightarrow \sigma_{q}^{*} M_{q}\left(G\left[z^{n}\right]\right)
$$

with

$$
\begin{aligned}
& F_{M_{q}\left(G\left[z^{n}\right]\right)} \circ V_{M_{q}\left(G\left[z^{n}\right]\right)}=(z-\zeta)^{d} \cdot \operatorname{id}_{M_{q}\left(G\left[z^{n}\right]\right),} \\
& V_{M_{q}\left(G\left[z^{n}\right]\right)} \circ F_{M_{q}\left(G\left[z^{n}\right]\right)}=(z-\zeta)^{d} \cdot \operatorname{id}_{\sigma_{q}^{*} M_{q}\left(G\left[z^{n}\right]\right)} .
\end{aligned}
$$

Under the identification of the co-Lie complex $\ell_{G\left[z^{n}\right] / S}^{\bullet}$ with

$$
0 \longrightarrow \sigma_{q}^{*} M_{q}\left(G\left[z^{n}\right]\right) \stackrel{F_{M_{q}\left(G\left[z^{n}\right]\right)}}{\longrightarrow} M_{q}\left(G\left[z^{n}\right]\right) \longrightarrow 0
$$

from Theorem 5.2 (iii)(d) the map $V_{M_{q}\left(G\left[z^{n}\right]\right)}$ corresponds to a homotopy $h: t_{G\left[z^{n}\right]}^{*} \rightarrow$ $N_{G\left[z^{n}\right]}$ with $d h=(z-\zeta)^{d}$ on $t_{G\left[z^{n}\right]}^{*}$ and $h d=(z-\zeta)^{d}$ on $N_{G\left[z^{n}\right]}$. This means that $(z-\zeta)^{d}$ is homotopic to zero on $\ell_{G\left[z^{n}\right] / S}^{\bullet}$.

Proposition 9.6 Assume that $\zeta=0$ in $\mathcal{O}_{s}$. Let $G=G\left[z^{l}\right]$ be a truncated $z$-divisible local Anderson module over $S$ with order of nilpotence $d$ and level $l$.

(i) There exists a morphism $V_{z^{d}, G}: \sigma_{q}^{*} G \rightarrow G$ with $F_{q, G} \circ V_{z^{d}, G}=z^{d} \cdot \operatorname{id}_{\sigma_{q}^{*} G}$ and $V_{z^{d}, G} \circ F_{q, G}=z^{d} \cdot \mathrm{id}_{G}$. It is not uniquely determined, unless $G$ is étale.

(ii) $G\left[F_{q}^{i}\right] \subset G\left[z^{i d}\right]$ and $\operatorname{ker} V_{z^{d}, G}^{i} \subset \sigma_{q^{i}}^{*} G\left[z^{i d}\right]$ for all $i$.

Now let $n \in \mathbb{N}_{>0}$ and $l=n d$. In particular, if $n=1$, there is a truncated divisible local Anderson module $\widetilde{G}$ of level $2 d$ with $G=\widetilde{G}\left[z^{d}\right]$ and we assume that $V_{z^{d}, G}=\left.V_{z^{d}, \widetilde{G}}\right|_{\sigma_{q}^{*} G}$. Then the following hold. 
(iii) For all $i$, with $0 \leq i \leq n$ the morphism $F_{q, G}^{i}: G\left[F_{q}^{n}\right] \rightarrow \sigma_{q^{i}}^{*} G\left[F_{q}^{n-i}\right]$ is an epimorphism.

(iv) The morphisms $V_{z^{d}, G}^{n}: \sigma_{q^{n}}^{*} G \rightarrow \operatorname{ker} F_{q, G}^{n}$ and $F_{q, G}^{n}: G \rightarrow \operatorname{ker} V_{z^{d}, G}^{n}$ are epimorphisms.

(v) $G\left[F_{q}^{i}\right]$ and ker $V_{z^{d}, G}^{i}$ are finite locally free strict $\mathbb{F}_{q}$-module schemes over $S$ for all $0 \leq i \leq n$.

(vi) For all $0 \leq i \leq n$, we have $\omega_{G}=\omega_{G\left[z^{i d}\right]}=\omega_{G\left[F_{q}\right]}$ and this is a finite locally free $\mathcal{O}_{S}-$ module.

Proof (i) Let $h: t_{G}^{*} \rightarrow N_{G}$ be a homotopy satisfying $d h=(z-\zeta)^{d}$ on $t_{G}^{*}$ and also $h d=(z-\zeta)^{d}$ on $N_{G}$. Note that $h$ is determined only up to adding a homomorphism $t_{G}^{*} \rightarrow \operatorname{coker} d=\omega_{G} \rightarrow n_{G}=\operatorname{ker} d \hookrightarrow N_{G}$, and in particular, is not unique unless $G$ is étale. Let $V: M_{q}(G) \rightarrow \sigma_{q}^{*} M_{q}(G)$ be the homomorphism that corresponds to $h$ under the identification of the co-Lie complex $\ell_{G / S}^{\bullet}$ with

$$
0 \rightarrow \sigma_{q}^{*} M_{q}(G) \stackrel{F_{M_{q}(G)}}{\longrightarrow} M_{q}(G) \rightarrow 0
$$

from Theorem $5.2(\mathrm{iii})(\mathrm{d})$. Then $V \circ F_{M_{q}(G)}=z^{d} \cdot \operatorname{id}_{\sigma_{q}^{*} M_{q}(G)}=\sigma_{q}^{*}\left(z^{d} \cdot \operatorname{id}_{M_{q}(G)}\right)=$ $\sigma_{q}^{*}\left(F_{M_{q}(G)} \circ V\right)=F_{\sigma_{q}^{*} M_{q}(G)} \circ \sigma_{q}^{*} V$ implies that $V: \underline{M}_{q}(G) \rightarrow \sigma_{q}^{*} \underline{M}_{q}(G)=\underline{M}_{q}\left(\sigma_{q}^{*} G\right)$ is a morphism of finite $\mathbb{F}_{q}$-shtukas. It induces the desired morphism

$$
V_{z^{d}, G}:=\operatorname{Dr}_{q}(V): \sigma_{q}^{*} G \rightarrow G
$$

with $F_{q, G} \circ V_{z^{d}, G}=z^{d} \cdot \operatorname{id}_{\sigma_{q}^{*} G}$ and $V_{z^{d}, G} \circ F_{q, G}=z^{d} \cdot \operatorname{id}_{G}$.

(ii) follows from $V_{z^{d}, G}^{i} \circ F_{q, G}^{i}=z^{i d} \cdot \operatorname{id}_{G}$ and $F_{q, G}^{i} \circ V_{z^{d}, G}^{i}=z^{i d} \cdot \operatorname{id}_{\sigma_{q^{i}}^{*} G}$ which are consequences of (i).

(iii) is trivial if $n=1$ and $i=0$ or 1 . If $n \geq 2$, there is by (i) a factorization $F_{q, G}^{i}$ 。 $V_{z^{d}, G}^{i}=z^{i d}: \sigma_{q^{i}}^{*} G \rightarrow \sigma_{q^{i}}^{*} G$. Since the morphism $z^{i d}: \sigma_{q^{i}}^{*} G \rightarrow \sigma_{q^{i}}^{*} G\left[z^{(n-i) d}\right]$ is an epimorphism by Lemma 7.8, and since $\sigma_{q^{i}}^{*} G\left[F_{q}^{n-i}\right] \subset \sigma_{q^{i}}^{*} G\left[z^{(n-i) d}\right]$ by (ii), we obtain (iii).

(iv) is proved by induction on $n$. For $n=1$, we use $G=\widetilde{G}\left[z^{d}\right]$. By Lemma 7.8 there is an exact sequence $0 \rightarrow \widetilde{G}\left[z^{d}\right] \rightarrow \widetilde{G} \stackrel{z^{d}}{\rightarrow} \widetilde{G}\left[z^{d}\right] \rightarrow 0$. Since $G\left[F_{q}\right] \subset \widetilde{G}\left[F_{q}\right] \subset$ $\widetilde{G}\left[z^{d}\right]$ by (ii), the map $V_{z^{d}, \widetilde{G}} \circ F_{q, \widetilde{G}}=z^{d}:\left(z^{d}\right)^{-1}\left(G\left[F_{q}\right]\right) \rightarrow G\left[F_{q}\right]$ is an epimorphism. From $F_{q, \widetilde{G}}:\left(z^{d}\right)^{-1}\left(G\left[F_{q}\right]\right) \rightarrow \sigma_{q}^{*} \widetilde{G}\left[z^{d}\right]=\sigma_{q}^{*} G$ we see that $V_{z^{d}, G}=\left.V_{z^{d}, \widetilde{G}}\right|_{\sigma_{q}^{*} G}: \sigma_{q}^{*} G \rightarrow$ $G\left[F_{q}\right]$ is an epimorphism. The statement for $F_{q, G}$ is proved in the analogous way using ker $V_{z^{d}, G} \subset \operatorname{ker} V_{z^{d}, \widetilde{G}} \subset \sigma_{q}^{*} \widetilde{G}\left[z^{d}\right]$. Thus we have proved (iv) for the case $n=1$.

To prove it in general by induction on $n$, consider the diagram

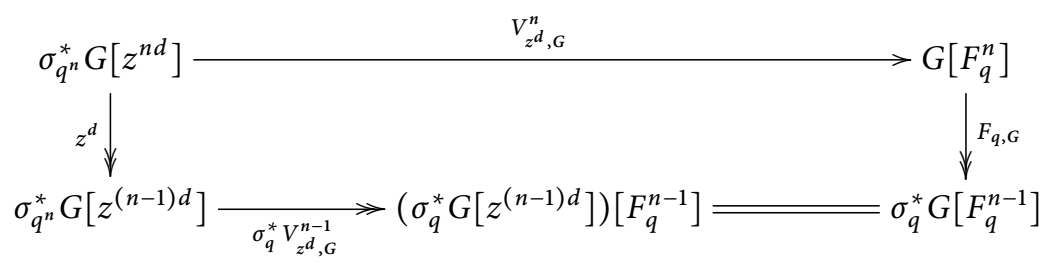


In the bottom row, $\sigma_{q}^{*} V_{z^{d}, G}^{n-1}$ is an epimorphism by the induction hypothesis, and the equality comes from (ii). The vertical map on the left is an epimorphism by Lemma 7.8, and therefore $F_{q, G} \circ V_{z^{d}, G}^{n}$ is an epimorphism. Thus if we can show that $\operatorname{ker}\left(F_{q, G}\right)=$ $G\left[F_{q}\right]$ is contained in the image of $V_{z^{d}, G}^{n}$, it will follow that $V_{z^{d}, G}^{n}$ is an epimorphism. But by the case $n=1$ settled above

$$
\begin{aligned}
G\left[F_{q}\right] & =V_{z^{d}, G}\left(\sigma_{q}^{*} G\left[z^{d}\right]\right)=V_{z^{d}, G} \circ z^{(n-1) d}\left(\sigma_{q}^{*} G\left[z^{n d}\right]\right) \\
& =V_{z^{d}, G}^{n} \circ F_{q, G}^{n-1}\left(\sigma_{q}^{*} G\left[z^{n d}\right]\right) \subset V_{z^{d}, G}^{n}\left(\sigma_{q^{n}}^{*} G\left[z^{n d}\right]\right) .
\end{aligned}
$$

This proves that $V_{z^{d}, G}^{n}$ is an epimorphism. The statement for $F_{q, G}^{n}$ is proved in the same way.

(v) The morphisms $F_{q, G}^{i}: G \rightarrow \sigma_{q^{i}}^{*} G$ and $V_{z^{d}, G}^{i}: \sigma_{q^{i}}^{*} G \rightarrow G$ between group schemes of finite presentation over $S$ are themselves of finite presentation by [EGA, IV ${ }_{1}$, Proposition 1.6.2(v)]. Therefore $G\left[F_{q}^{i}\right]:=\operatorname{ker} F_{q, G}^{i}$ and $\operatorname{ker} V_{z^{d}, G}^{i}$ are of finite presentation over $S$ by [EGA, $I_{1}$, Proposition 1.6.2(iii)]. As closed subschemes of $G$, respectively $\sigma_{q^{i}}^{*} G$, they are also finite over $S$. Since in (iv) we proved that

$$
V_{z^{d}, G}^{i}: \sigma_{q^{i}}^{*} G\left[z^{i d}\right] \rightarrow\left(G\left[z^{\mathrm{id}}\right]\right)\left[F_{q}^{i}\right]=G\left[F_{q}^{i}\right]
$$

and

$$
F_{q, G}^{i}: G\left[z^{\mathrm{id}}\right] \rightarrow \operatorname{ker} V_{z^{d}, G\left[z^{i d}\right]}^{i}=\operatorname{ker} V_{z^{d}, G}^{i}
$$

are epimorphisms, they are faithfully flat by Remark $3.1(\mathrm{~b})$. Therefore $G\left[F_{q}^{i}\right]$ and $\operatorname{ker} V_{z^{d}, G}^{i}$ are flat over $S$ by [EGA, $\mathrm{IV}_{3}$, Corollaire 11.3.11], and hence finite locally free. Over any affine open $U \subset S$ the $\mathbb{F}_{q}$-equivariant morphisms $F_{q, G}^{i}$ and $V_{z^{d}, G}^{i}$ lift by Lemma 4.6 to morphisms in $\operatorname{DGr}\left(\mathbb{F}_{q}\right)_{U}$. Thus they are $\mathbb{F}_{q}$-strict morphisms in the sense of Faltings [Fal02, Definition 1]. By [Fal02, Proposition 2], their kernels

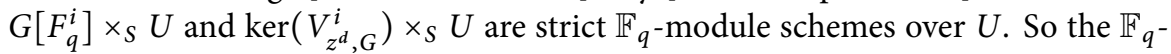
strictness of $G\left[F_{q}^{i}\right]$ and $\operatorname{ker} V_{z^{d}, G}^{i}$ over all of $S$ follows from Lemma 4.8.

(vi) For any group scheme $G=\operatorname{Spec} R\left[X_{1}, \ldots, X_{r}\right] / I$ of finite type over $\operatorname{Spec} R$, we compute $G\left[F_{q}\right]=\operatorname{Spec} R\left[X_{1}, \ldots, X_{r}\right] /\left(I, X_{1}^{q}, \ldots, X_{r}^{q}\right)$. By the conormal sequence [Har77, Proposition II.8.12] for the closed immersion $G\left[F_{q}\right] \subset G$, this implies that

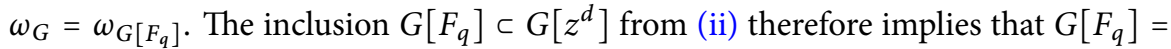
$\left(G\left[z^{\text {id }}\right]\right)\left[F_{q}\right]$, and hence $\omega_{G}=\omega_{G\left[z^{\text {id }}\right]}=\omega_{G\left[F_{q}\right]}$ for all $i$. Moreover, since $G\left[F_{q}\right]$ is a finite locally free strict $\mathbb{F}_{q}$-module scheme over $S$ by $(\mathrm{v})$, we can compute $\omega_{G\left[F_{q}\right]}$ as coker $F_{M_{q}\left(G\left[F_{q}\right]\right)}$, where $\left(M_{q}\left(G\left[F_{q}\right]\right), F_{M_{q}\left(G\left[F_{q}\right]\right)}\right)$ is the associated finite $\mathbb{F}_{q}$-shtuka from Theorem 5.2. In particular, $F_{M_{q}\left(G\left[F_{q}\right]\right)}=\underline{M}_{q}\left(F_{q, G\left[F_{q}\right]}\right)=0$ and this implies that coker $F_{M_{q}\left(G\left[F_{q}\right]\right)}=M_{q}\left(G\left[F_{q}\right]\right)$ is a finite locally free $\mathcal{O}_{S}$-module.

In the remainder of this section we will show that to lift a $z$-divisible local Anderson-module is equivalent to lifting its Hodge filtration. Let $S \in \mathcal{N i l p}_{\left.\mathbb{F}_{q}[\zeta]\right]}$ and let $G$ be a $z$-divisible local Anderson module over $S$ satisfying $(z-\zeta)^{d} \cdot \omega_{G}=0$. Let $\left(M, F_{M}\right)$ be its effective local shtuka. Then $(z-\zeta)^{d} \cdot \operatorname{coker} F_{M}=0$ and we consider the map $V_{M}$ from Lemma 9.2. The injective morphism $F_{M}$ induces by diagram (9.1) an exact sequence of $\mathcal{O}_{S}[[z]]$-modules

$$
0 \longrightarrow \operatorname{coker} V_{M} \stackrel{F_{M}}{\longrightarrow} M /(z-\zeta)^{d} M \longrightarrow \operatorname{coker} F_{M} \longrightarrow 0 .
$$


In particular coker $V_{M}$ is a locally free $\mathcal{O}_{S}$-module of finite rank. Conversely, $V_{M}$ induces the exact sequence of $\mathcal{O}_{S}[[z]]$-modules

$$
0 \longrightarrow \operatorname{coker} F_{M} \stackrel{V_{M}}{\longrightarrow} \sigma_{q}^{*} M /(z-\zeta)^{d} \sigma_{q}^{*} M \longrightarrow \operatorname{coker} V_{M} \longrightarrow 0 .
$$

Definition 9.7 (cf. [HJ19, §5.7]) We call

$$
H(G):=\mathrm{H}_{\mathrm{dR}}^{1}\left(G, \mathcal{O}_{S}[z] /(z-\zeta)^{d}\right):=\sigma_{q}^{*} M /(z-\zeta)^{d} \sigma_{q}^{*} M
$$

the de Rham cohomology of $G$ with coefficients in $\mathcal{O}_{S}[z] /(z-\zeta)^{d}$. It is a locally free $\mathcal{O}_{S}[z] /(z-\zeta)^{d}$-module of rank equal to $\operatorname{rk} \underline{M}=$ height $G$. The $\mathcal{O}_{S}[z]$-submodule $V_{M}\left(\right.$ coker $\left.F_{M}\right) \subset H(G)$ is called the Hodge filtration of the $z$-divisible local Anderson module $G$.

Now let $i: S^{\prime} \hookrightarrow S$ be a closed subscheme defined by an ideal $I$ with $I^{q}=0$. Then the morphisms Frob F,s and Frob $\mathrm{b}_{q, S^{\prime}}$ factor through $i$

$$
\operatorname{Frob}_{q, S}=i \circ j: S \rightarrow S^{\prime} \rightarrow S \text { and } \text { Frob }_{q, S^{\prime}}=j \circ i: S^{\prime} \rightarrow S \rightarrow S^{\prime},
$$

where $j: S \rightarrow S^{\prime}$ is the identity on the underlying topological space $\left|S^{\prime}\right|=|S|$ and on the structure sheaf this factorization is given by

$$
\begin{aligned}
& \mathcal{O}_{S} \stackrel{i^{*}}{\rightarrow} \quad \mathcal{O}_{S^{\prime}} \quad \stackrel{j^{*}}{\rightarrow} \mathcal{O}_{S} \\
& b \mapsto b \bmod I \mapsto b^{q} \text {. }
\end{aligned}
$$

Let $G^{\prime}$ be a divisible local Anderson module over $S^{\prime}$ with $(z-\zeta)^{d} \cdot \omega_{G^{\prime}}=0$, and denote by $\left(M^{\prime}, F_{M^{\prime}}\right)$ its local shtuka. We set $H\left(G^{\prime}\right)_{S}:=j^{*} M^{\prime} /(z-\zeta)^{d} j^{*} M^{\prime}$. This is a locally free module over $\mathcal{O}_{S}[z] /(z-\zeta)^{d}$ and satisfies $i^{*} H\left(G^{\prime}\right)_{S}=H\left(G^{\prime}\right)$.

Theorem 9.8 The functor $G \mapsto\left(i^{*} G, V_{M}\left(\right.\right.$ coker $\left.\left.F_{M}\right) \subset H(G)\right)$ defines an equivalence between

(i) the category of $z$-divisible local Anderson modules $G$ over $S$ with $(z-\zeta)^{d} \cdot \omega_{G}=0$, and

(ii) the category of pairs $\left(G^{\prime}, \mathrm{Fil} \subset H\left(G^{\prime}\right)_{S}\right)$, where $G^{\prime}$ is a $z$-divisible local Anderson module over $S^{\prime}$ and Fil $\subset H\left(G^{\prime}\right)_{S}$ is an $\mathcal{O}_{S}[[z]]$-submodule whose quotient is a flat $\mathcal{O}_{S}$-module that specializes to the $\mathcal{O}_{S}^{\prime}[[z]]$-submodule $V_{M^{\prime}}\left(\operatorname{coker} F_{M^{\prime}}\right) \subset H\left(G^{\prime}\right)$ under $i$.

Proof We describe the quasi-inverse functor. Let $\left(G^{\prime}\right.$, Fil $\left.\subset H\left(G^{\prime}\right)_{S}\right)$ be given and let $\left(M^{\prime}, F_{M^{\prime}}\right)$ be the local shtuka of $G^{\prime}$. We define $V_{M}: M \hookrightarrow j^{*} M^{\prime}$ as the kernel of the morphism $j^{*} M^{\prime} \rightarrow H\left(G^{\prime}\right)_{S} /$ Fil. Since Fil $\subset H\left(G^{\prime}\right)_{S}$ specializes to $V_{M}\left(\operatorname{coker} F_{M^{\prime}}\right) \subset$ $H\left(G^{\prime}\right)$ we obtain $i^{*}\left(H\left(G^{\prime}\right)_{S} /\right.$ Fil $)=H\left(G^{\prime}\right) / V_{M^{\prime}}\left(\operatorname{coker} F_{M^{\prime}}\right)=$ coker $V_{M^{\prime}}$. This implies $i^{*} M \cong M^{\prime}$ and $\sigma_{q}^{*} M=j^{*} i^{*} M \cong j^{*} M^{\prime}$. Moreover coker $V_{M}$ is annihilated by $(z-\zeta)^{d}$. Thus there is an injective morphism of $\mathcal{O}_{S}\left[[z]\right.$-modules $F_{M}: \sigma_{q}^{*} M \rightarrow M$ with $F_{M} V_{M}=(z-\zeta)^{d} \mathrm{id}_{M}$ and $V_{M} F_{M}=(z-\zeta)^{d} \operatorname{id}_{\sigma_{q}^{*} M}$. From sequence (9.2) we see that the cokernel of $F_{M}$ is a locally free $\mathcal{O}_{S}$-module. Clearly the $z$-divisible local Anderson module $G$ over $S$ associated with the local shtuka $\left(M, F_{M}\right)$ specializes to $G^{\prime}$ and has Fil $\subset H\left(G^{\prime}\right)_{S}=H(G)$ as its Hodge filtration. 
Remark 9.9 We only treated the case where $S^{\prime} \subset S$ is defined by an ideal $I$ with $I^{q}=0$. The general case for $d=1$ is treated by Genestier and Lafforgue [GL11, Proposition 6.3] using $\zeta$-divided powers in the style of Grothendieck and Berthelot.

\section{Divisible Local Anderson Modules and Formal Lie Groups}

In this section we clarify the relation between $z$-divisible local Anderson modules and formal $\mathbb{F}_{q}[[z]]$-modules; see Definition 1.1. We follow the approach of Messing [Mes72] who treated the analogous situation of $p$-divisible groups and formal Lie groups.

Definition 10.1 Let $G$ be an fppf-sheaf of abelian groups over $S \in \mathcal{N i l p}_{\mathbb{F}_{q}[\zeta \zeta]}$. We say that $G$ is F-torsion if $G=\lim _{\longrightarrow} G\left[F_{q}^{n}\right]$, and that $G$ is F-divisible if $F_{q, G}: G \rightarrow \sigma_{q}^{*} G$ is an epimorphism.

Recall that Messing [Mes72, Chapter II, Theorem 2.1.7] proved that a sheaf of groups $G$ on $S$ is a formal Lie group [Mes72, Chapter II, Definitions 1.1.4, 1.1.5], if and only if $G$ is $F$-torsion, $F$-divisible, and the $G\left[F_{q}^{n}\right]$ are finite locally free $S$-group schemes.

Theorem 10.2 When $\zeta=0$ in $\mathcal{O}_{S}$ and $G$ is a $z$-divisible local Anderson module over $S$, then $\underset{\vec{n}}{\lim } G\left[F_{q}^{n}\right]$ is a formal $\mathbb{F}_{q} \llbracket[z]$-module. It is equal to $\bar{G}:=\underset{\vec{k}}{\lim } m \operatorname{Inf}^{k}(G)$, where for any S-scheme T, Messing [Mes72, Chapter II, (1.1)] defined

$$
\begin{gathered}
\left(\operatorname{Inf}^{k} G\right)(T):=\left\{x \in G(T): \text { there is an fppf-covering }\left\{\operatorname{Spec} R_{i} \rightarrow T\right\}_{i}\right. \text { and } \\
\text { for every } i \text { an ideal } I_{i} \subset R_{i} \text { with } I_{i}^{k+1}=(0) \text { such that the } \\
\text { pull-back } \left.x \in G\left(\operatorname{Spec} R_{i} / I_{i}\right) \text { is zero }\right\}
\end{gathered}
$$

Proof By [Mes72, Chapter II, Theorem 2.1.7] it suffices to show that $\underset{\longrightarrow}{\longrightarrow} G\left[F_{q}^{n}\right]$ is $F$-torsion, $F$-divisible, and that the $G\left[F_{q}^{n}\right]$ are finite locally free. By construction $\underset{\lim }{\longrightarrow}\left[F_{q}^{n}\right]$ is $F$-torsion. By Definition 7.1(iv) there is locally on $S$ an integer $d$ with

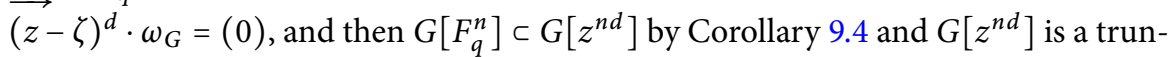
cated $z$-divisible local Anderson module with order of nilpotence $d$ and level $n d$ by Proposition 9.5. Therefore Proposition 9.6 shows that $G\left[F_{q}^{n}\right]$ is finite locally free, and that $F_{q, G}: G\left[F_{q}^{n}\right] \rightarrow \sigma_{q}^{*} G\left[F_{q}^{n-1}\right]$ is an epimorphism. Consequently,

$$
F_{q, G}: \lim _{\longrightarrow} G\left[F_{q}^{n}\right] \rightarrow \sigma_{q}^{*}\left(\lim _{\longrightarrow} G\left[F_{q}^{n}\right]\right)=\lim _{\longrightarrow} \sigma_{q}^{*} G\left[F_{q}^{n-1}\right]
$$

is an epimorphism and so $\lim _{\longrightarrow} G\left[F_{q}^{n}\right]$ is F-divisible, and hence a formal Lie group. The action of $\mathbb{F}_{q}[[z]]$ makes it into a formal $\mathbb{F}_{q}[[z]]$-module.

To prove the last statement of the theorem, observe that for any $S$-scheme $T$, the homomorphism $F_{q^{n}, G}: G(T) \rightarrow\left(\sigma_{q^{n}}^{*} G\right)(T)$ is simply the map sending $x$ to 
$x \circ \operatorname{Frob}_{q^{n}, T}$ as can be seen from the following diagram:

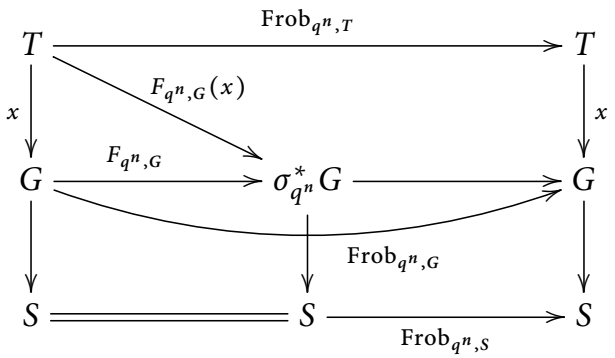

Therefore, the monomorphism $G\left[F_{q}^{n}\right] \hookrightarrow G$ defines an inclusion $G\left[F_{q}^{n}\right] \subset \operatorname{Inf}^{q^{n}-1} G$, the ideals $I_{i}$ in (10.1) being the augmentation ideal in $\mathcal{O}_{G\left[F_{q}^{n}\right]}$ defining the zero section. We claim that this inclusion is an equality. So let $x \in\left(\operatorname{Inf}^{q^{n}-1} G\right)(T)$ and let $R_{i}$ and $I_{i}$ be as in (10.1). Then $I_{i}^{q^{n}}=(0)$ implies that Frob $_{q^{n}, R_{i}}$ factors through $R_{i} \rightarrow R_{i} / I_{i} \stackrel{j}{\rightarrow} R_{i}$. So $\left.F_{q^{n}, G}(x)\right|_{\operatorname{Spec} R_{i}}=x \circ \operatorname{Frob}_{q^{n}, R_{i}}=j^{*}\left(\left.x\right|_{\operatorname{Spec} R_{i} / I_{i}}\right)=0$, that is, $x \in G\left[F_{q}^{n}\right]$. Thus we have $G\left[F_{q}^{n}\right]=\operatorname{Inf}^{q^{n}-1} G$ and $\lim _{\longrightarrow} G\left[F_{q}^{n}\right]=\lim _{\longrightarrow} \operatorname{Inf}^{k}(G) \subset G$ which completes the proof.

Our next aim is to extend the theorem to all $S \in \mathcal{N i l p}_{\left.\mathbb{F}_{q} \llbracket \zeta\right]}$. For that purpose we start with the following lemma.

Lemma 10.3 Let $S$ be a scheme with $\zeta^{N+1}=0$ in $\mathcal{O}_{S}$, and let $G=G\left[z^{n d}\right]$ be a truncated $z$-divisible local Anderson module over $S$ with order of nilpotence $d$ and level $n d$ with $n \geq N+1$. Then for any affine open subset $U$ of $S$ and any quasicoherent sheaf $\mathcal{F}$ of $\mathcal{O}_{U}$-modules, the natural homomorphism for the co-Lie complexes $\operatorname{Ext}_{\mathcal{O}_{U}}^{1}\left(\ell_{G\left[z^{(n-N-1) d}\right] / U}^{\bullet}, \mathcal{F}\right) \rightarrow \operatorname{Ext}_{\mathcal{O}_{U}}^{1}\left(\ell_{G\left[z^{n d}\right] / U}^{\bullet}, \mathcal{F}\right)$ is zero.

Proof We proceed by induction on $N$ and begin with $N=0$. If $n=1$, then

$$
G\left[z^{(n-N-1) d}\right]=(0)
$$

and there is nothing to prove. If $n \geq 2$, we use [Mes72, Chapter II, Corollary 3.3.9] for the sequence $0 \rightarrow G\left[z^{(n-1) d}\right] \rightarrow G\left[z^{n d}\right] \stackrel{z^{(n-1) d}}{\longrightarrow} G\left[z^{d}\right] \rightarrow 0$. So we must show that $\omega_{G\left[z^{n d}\right]} \rightarrow \omega_{G\left[z^{(n-1) d}\right]}$ is an isomorphism, that $\omega_{G\left[z^{n d}\right]}$ and $\omega_{G\left[z^{d}\right]}$ are locally free $\mathcal{O}_{S}$-modules, and that $\mathrm{rk} \omega_{s^{*} G\left[z^{(n-1) d}\right]} \leq \operatorname{rk} \omega_{s^{*} G\left[z^{d}\right]}$ for all points $s \in S$. All three statements follow from Proposition 9.6(vi). This concludes the proof when $N=0$. 
For general $N$ we take the exact sequence $0 \rightarrow \zeta \mathcal{F} \rightarrow \mathcal{F} \rightarrow \mathcal{F} / \zeta \mathcal{F} \rightarrow 0$. and consider the following commutative diagram with exact rows.

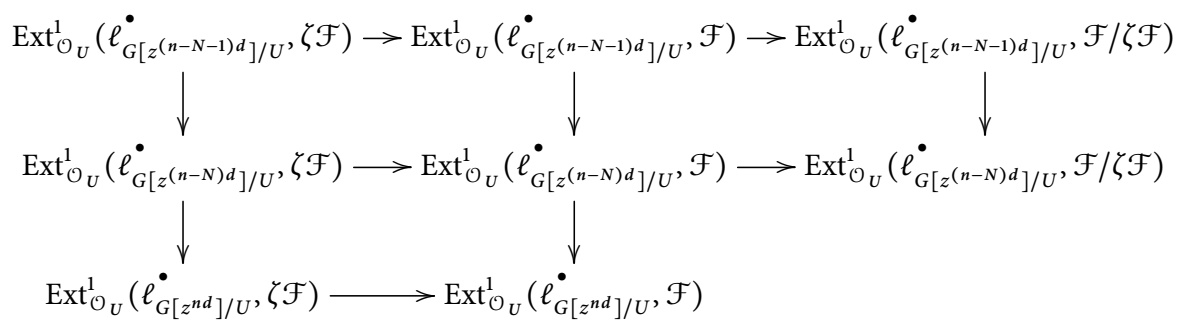

Since $\zeta \cdot(\mathcal{F} / \zeta \mathcal{F})=(0)$, the right vertical arrow can be computed by base change to the zero locus $\mathrm{V}(\zeta) \subset S$ of $\zeta$. So it is the zero map by what we have proved above, and hence the image of $\operatorname{Ext}_{\mathcal{O}_{U}}^{1}\left(\ell_{G_{\left[z^{(n-N-1) d}\right] / U}}, \mathcal{F}\right)$ in $\operatorname{Ext}_{\mathcal{O}_{U}}^{1}\left(\ell_{G_{\left[z^{(n-N) d}\right] / U}}^{\bullet}, \mathcal{F}\right)$ is contained in the image of $\operatorname{Ext}_{\mathcal{O}_{U}}^{1}\left(\ell_{G\left[z^{(n-N) d}\right] / U}^{\bullet}, \zeta \mathcal{F}\right)$. Since $\zeta^{N} \cdot(\zeta \mathcal{F})=(0)$, the lower left vertical arrow can similarly be computed by base change to the zero locus $\mathrm{V}\left(\zeta^{N}\right) \subset S$, and hence it is the zero map by our induction hypothesis. This proves the lemma.

Theorem 10.4 If $S \in \mathcal{N i l p}_{\mathbb{F}_{q}[[\zeta]]}$ and $G$ is a $z$-divisible local Anderson module over $S$, then $G$ is formally smooth.

Proof Let $X^{\prime}$ be an affine scheme over $S$ and let $X$ be a closed subscheme defined by an ideal of square zero. Let $f: X \rightarrow G$ be an $S$-morphism. We must show that $f$ can be lifted to an $S$-morphism $f^{\prime}: X^{\prime} \rightarrow G$. As $X$ is quasi-compac,t we have $G(X)=$ $\underset{n}{\lim _{\vec{n}}} G\left[z^{n}\right](X)=\underset{n}{\lim _{\vec{n}}} G\left[z^{n d}\right](X)$, and hence $f: X \rightarrow G\left[z^{\text {nd }}\right]$ for some $n$ by Remark 7.2. We cover $X$ by a finite number of affine opens $U_{i}, i=1, \ldots, m$ such that the image of $U_{i}$ in $S$ is contained in an affine open $V_{i}$. Since $\zeta$ is nilpotent on each $V_{i}$, there is an integer $N$ such that $\zeta^{N+1}$ is zero on $\bigcup V_{i}$. Replacing $S$ by $S^{\prime}=\cup V_{i}$ and $G$ by $G_{S^{\prime}}$ we are led to the case where $\zeta^{N+1}=0$ in $\mathcal{O}_{S}$. But now Lemma 10.3 and [Mes72, Chapter II, Proposition 3.3.1] show that $f$ can be lifted to an $f^{\prime}: X^{\prime} \rightarrow G\left[z^{(n+N+1) d}\right]$, and the theorem is proved.

Lemma 10.5 Let $G$ be a $z$-divisible local Anderson module over $S$ with $(z-\zeta)^{d}=0$ on $\omega_{G}$ for some $d \in \mathbb{N}$. Assume we are given an $S$-scheme $X^{\prime}$ and a subscheme $X$ defined by a sheaf of ideals $I$ such that $I^{k+1}=(0)$ and $\zeta^{N} \cdot I / I^{2}=0$ for some integer $N$. Let $N^{\prime}$ be the smallest integer that is a power of $p$ and greater or equal to $N$ and $d$. If an S-morphism $f^{\prime}: X^{\prime} \rightarrow G$ satisfies $f=\left.f^{\prime}\right|_{X}: X \rightarrow G\left[z^{n}\right]$, then $f^{\prime}$ factors through $f^{\prime}: X^{\prime} \rightarrow G\left[z^{n+k N^{\prime}}\right] \subset G$.

Proof The problem is local on $X^{\prime}$ and hence we can assume that $X^{\prime}$ is affine and thus quasi-compact. But then $f^{\prime} \in G\left(X^{\prime}\right)=\lim _{\longrightarrow} G\left[z^{m}\right]\left(X^{\prime}\right)$ and hence we can assume that $f^{\prime}: X^{\prime} \rightarrow G\left[z^{n^{\prime}}\right]$ for some $n^{\prime}$ by Remark 7.2. We now use induction on $k$ and the sequence of closed subschemes $\mathrm{V}\left(I^{l}\right) \subset X^{\prime}$ for $l=1, \ldots, k+1$. Thus we can assume that $I^{2}=0$ and $k=1$. 
Since $f \in G\left[z^{n}\right](X)$, we have $z^{n} f=0$, and so $z^{n} f^{\prime} \in G\left[z^{n^{\prime}}\right]\left(X^{\prime}\right)$ has the property that its restriction to $G\left[z^{n^{\prime}}\right](X)$ is zero. Since $I^{2}=0$, the group of sections of $G\left[z^{n^{\prime}}\right]$ over $X^{\prime}$ whose restriction to $X$ is zero, is, by [SGA 3, III, Théorème 0.1.8(a)], isomorphic to the group $\operatorname{Hom}_{\mathcal{O}_{X}}\left(\omega_{G\left[z^{n^{\prime}}\right]} \otimes_{\mathcal{O}_{S}} \mathcal{O}_{X}, I\right)$ under an isomorphism that sends the zero morphism $X^{\prime} \rightarrow G\left[z^{n^{\prime}}\right]$ to the zero element, and the morphism $z^{n} f^{\prime}$ to an element that we denote by $h \in \operatorname{Hom}_{\mathcal{O}_{X}}\left(\omega_{G} \otimes_{\mathcal{O}_{S}} \mathcal{O}_{X}, I\right)$. Since $\zeta^{N}$ kills $I$ and $N^{\prime} \geq N$, we obtain $\zeta^{N^{\prime}} \cdot h=0$. On $\omega_{G}$ the assumption $(z-\zeta)^{d}=0$ implies $z^{N^{\prime}}=\zeta^{N^{\prime}}$, and so the section $z^{N^{\prime}}\left(z^{n} f^{\prime}\right)$ is sent to $z^{N^{\prime}} \cdot h=\zeta^{N^{\prime}} \cdot h=0$. This implies $z^{n+N^{\prime}} f^{\prime}=0$, that is, $f^{\prime} \in G\left[z^{n+N^{\prime}}\right]\left(X^{\prime}\right)$.

Corollary 10.6 Let $\zeta^{N}=0$ in $\mathcal{O}_{S}$ and let $G$ and $d$ be as in Lemma 10.5. Let $N^{\prime}$ be the smallest integer that is a power of $p$ and greater or equal to $N$ and $d$. Then the $k$-th infinitesimal neighborhood of $G\left[z^{n}\right]$ in $G$ is the same as that of $G\left[z^{n}\right]$ in $G\left[z^{n+k N^{\prime}}\right]$. In particular, $\operatorname{Inf}^{k}(G)=\operatorname{Inf}^{k}\left(G\left[z^{k N^{\prime}}\right]\right)$ and this is therefore representable.

Proof By definition [Mes72, Chapter II, Definition (1.01)], an $S$-morphism $f: T^{\prime} \rightarrow$ $G$ belongs to the $k$-th infinitesimal neighborhood of $G\left[z^{n}\right]$ in $G$, if and only if there is an fppf-covering $\left\{\operatorname{Spec} R_{i} \rightarrow T^{\prime}\right\}_{i}$ and ideals $I_{i} \subset R_{i}$ with $I_{i}^{k+1}=(0)$ such that $\left.f\right|_{\text {Spec } R_{i} / I_{i}} \in G\left[z^{n}\right]\left(\operatorname{Spec} R_{i} / I_{i}\right)$. But then $f \in G\left[z^{n+k N^{\prime}}\right]\left(T^{\prime}\right)$ by Lemma 10.5. The last statement is the special case with $n=0$.

Theorem 10.7 Let $G$ be a $z$-divisible local Anderson module over $S \in \mathcal{N i l p}_{\mathbb{F}_{q} \llbracket \zeta \rrbracket}$. Then $\bar{G}=\lim _{\longrightarrow} \operatorname{Inf}^{k}(G)$ is a formal $\mathbb{F}_{q}[[z]]$-module.

Proof As $\bar{G}$ clearly is an $\mathbb{F}_{q}[[z]$-submodule of $G$, we must show that it is a formal Lie variety; see [Mes72, Chapter II, Definition 1.1.4]. By construction it is indinfinitesimal. Since the question is local on $S$, we may assume that there are integers $N$ and $d$ as in Corollary 10.6. Then the sheaf $\operatorname{Inf}^{k}(G)$ is representable for all $k$. By Theorem 10.4 we know that $G$ is formally smooth and by definition (10.1) of $\operatorname{Inf}^{k}(G)$ this implies that $\bar{G}$ is formally smooth. Let $N^{\prime}$ be the smallest integer that is a power of $p$ and greater or equal to $N$ and $d$. Then $G\left[z^{k N^{\prime}}\right]$ satisfies the lifting condition 2) of [Mes72, Chapter II, Proposition 3.1.1] by Theorem 10.4 and Lemma 10.5. Therefore, by [Mes72, loc. cit] $G\left[z^{k N^{\prime}}\right]$ satisfies condition 2) and 3) of [Mes72, Chapter II, Definition 1.1.4], and hence is a formal $\mathbb{F}_{q}[[z]$-module.

Remark 10.8 We already know from Theorem 8.3 and Lemma 2.3 that $\omega_{G}$ is locally free of finite rank. This now follows again from the theorem because $\omega_{G}=\omega_{\bar{G}}$.

Next we pursue the question when a $z$-divisible local Anderson module is a formal $\mathbb{F}_{q}[[z]]$-module and vice versa.

Lemma 10.9 Let $B$ be a ring in which $\zeta$ is nilpotent, and let $I$ be a nilpotent ideal of $B$. Define a sequence of ideals $I_{1}:=\zeta I+I^{2}, \ldots, I_{n+1}:=\zeta I_{n}+\left(I_{n}\right)^{2}$. Then for $n$ sufficiently large $I_{n}=(0)$. 
Proof Let $J=\zeta B+I$. Then it is easy to check that $I_{n} \subset J^{n+1}$. Since $\zeta$ and $I$ are both nilpotent, so is the ideal $J$. This implies $I_{n}=0$ for $n$ sufficiently large.

Lemma 10.10 If $S \in \mathcal{N} \mathcal{N i l p}_{\mathbb{F}_{q} \llbracket \zeta \rrbracket}$ and $G$ is a formal $\left.\left.\mathbb{F}_{q} \llbracket z\right]\right]$-module over $S$ such that locally on $S$ there is an integer $d$ with $(z-\zeta)^{d}=0$ on $\omega_{G}$, then $G$ is $z$-torsion.

Proof We must show $G=\lim G\left[z^{n}\right]$ and since this is a statement about sheaves, it suffices to check it locally on $\vec{S}$. Thus we can assume $S=\operatorname{Spec} R$ with $\zeta \in R$ nilpotent and $G$ is given by a power series ring $R\left[\left[X_{1}, \ldots, X_{d}\right]\right]$; see [Mes72, p. 26]. If $T$ is any affine $S$-scheme, say $T=\operatorname{Spec} B$, then an element of $G(T)$ will be an $N$-tuple $\left(b_{1}, \ldots, b_{d}\right)$ with each $b_{i}$ nilpotent. Let $I$ be the ideal generated by $\left\{b_{1}, \ldots, b_{d}\right\}$. Let $N^{\prime}$ be a power of $p$ with $N^{\prime} \geq d$. Then multiplication with $z^{N^{\prime}}$ on $G$ is given by power series $\left(z^{N^{\prime}}\right)^{*}\left(X_{i}\right) \in R\left[\left[X_{1}, \ldots, X_{d}\right]\right]$ with linear term $\zeta^{N^{\prime}} X_{i}$ and without constant term. Therefore each component of $z^{N^{\prime}} .\left(b_{1}, \ldots, b_{d}\right)$ belongs to $\zeta^{N^{\prime}} I+I^{2} \subset \zeta I+I^{2}=: I_{1}$. Then each component of $z^{n N^{\prime}} \cdot\left(b_{1}, \ldots, b_{d}\right)$ belongs to the ideal $I_{n}$ from Lemma 10.9, and hence the lemma shows that $\left(b_{1}, \ldots, b_{d}\right)$ is $z$-torsion.

The next result is analogous to Messing's characterization [Mes72, Chapter II, Proposition 4.4] for a $p$-divisible group to be a formal Lie group, and its proof also follows similarly using Theorem 10.7.

Proposition 10.11 Let $S \in \mathcal{N i l p}_{\mathbb{F}_{q}[[\zeta]]}$ and let $G$ be a $z$-divisible local Anderson module over $S$. Then the following conditions are equivalent.

(i) $G=\bar{G}$.

(ii) $G$ is a formal $\mathbb{F}_{q}[[z]]$-module.

(iii) $G\left[z^{n}\right]$ is radicial for all $n$.

(iv) $G[z]$ is radicial.

Corollary 10.12 For $S \in \mathcal{N i l p}_{\mathbb{F}_{q} \llbracket \zeta \rrbracket}$, there is an equivalence of categories between that of $z$-divisible local Anderson modules over $S$ with $G[z]$ radicial and the category of $z$-divisible formal $\mathbb{F}_{q}[[z]$-modules $G$ with $G[z]$ representable by a finite locally free group scheme such that locally on $S$ there is an integer $d$ for which $(z-\zeta)^{d}=0$ on $\omega_{G}$.

Proof By Lemma 10.10 and Proposition 10.11, both categories are identified with the same full sub-category of fppf-sheaves of $\mathbb{F}_{q}[z]$-modules on $S$, once we observe that $G\left[z^{n}\right]:=\operatorname{ker}\left(z^{n}: G \rightarrow G\right)$ is a strict $\mathbb{F}_{q}$-module as the kernel of an $\mathbb{F}_{q}$-linear homomorphism of formal Lie groups that are $\mathbb{F}_{q}$-modules.

Corollary 10.13 Let $S \in \mathcal{N i l p}_{\left.\mathbb{F}_{q} \llbracket \zeta\right]}$ be the spectrum of an Artinian local ring. Then a $z$-divisible formal $\left.\mathbb{F}_{q} \llbracket z\right] \rrbracket$-module, such that locally on $S$ there is an integer $d$ for which $(z-\zeta)^{d}=0$ on $\omega_{G}$, is a $z$-divisible local Anderson module with $G[z]$ radicial and conversely. 
Proof This follows from Corollary 10.12 , because the $G\left[z^{n}\right]$ are automatically representable by finite locally free group schemes by [Mes72, Chapter II, Proposition 4.3].

The next result is analogous to Messing's characterization for a $p$-divisible group to be ind-étale, [Mes72, Chapter II, Proposition 4.7]. Its proof follows verbatim.

Proposition 10.14 Let $S \in \mathcal{N} \operatorname{Nil}_{\left.\mathbb{F}_{q} \llbracket \zeta\right]}$ and let $G$ be a $z$-divisible local Anderson module over $S$. In order that $\bar{G}=0$, it is necessary and sufficient that $G$ is (ind-)étale.

We have the following lemma for $z$-divisible local Anderson modules over $S$ similarly to and with the same proof as [Mes72, Chapter II, Proposition 4.11].

Lemma 10.15 Let $S \in \mathcal{N i l p}_{\mathbb{F}_{q}[[\zeta]]}$ and let $0 \rightarrow G_{1} \rightarrow G_{2} \rightarrow G_{3} \rightarrow 0$ be an exact sequence of $z$-divisible local Anderson modules over $S$. Then $0 \rightarrow \bar{G}_{1} \rightarrow \bar{G}_{2} \rightarrow \bar{G}_{3} \rightarrow 0$ is also exact.

Finally, there is a criterion when $\bar{G}$ is itself a $z$-divisible local Anderson module in analogy to Messing's criterion [Mes72, Chapter II, Proposition 4.9].

Proposition 10.16 Let $S \in \mathcal{N} \operatorname{Nilp}_{\mathbb{F}_{q}[[\zeta]]}$ and let $G$ be a z-divisible local Anderson module over $S$. Then the following conditions are equivalent.

(i) $\bar{G}$ is a $z$-divisible local Anderson module.

(ii) $G$ is an extension of an (ind-)étale $z$-divisible local Anderson module $G^{\prime \prime}$ by an ind-infinitesimal $z$-divisible local Anderson module $G^{\prime}$.

(iii) $G$ is an extension of an (ind-)étale $z$-divisible local Anderson module $G^{\prime \prime}$ by a $z$-divisible formal $\mathbb{F}_{q}[[z]]$-module $G^{\prime}$.

(iv) For all $n, G\left[z^{n}\right]$ is an extension of a finite étale group by a finite locally-free radicial group.

(v) $G[z]$ is an extension of a finite étale group by a finite locally-free radicial group.

(vi) The map $S \rightarrow \mathbb{Z}, s \mapsto \operatorname{ord}\left(G[z]_{s}\right)_{\text {ét }}=$ : separable rank $\left(G[z]_{s}\right)$ is a locally constant function on $S$.

Proof The proof proceeds in the same way as [Mes72, Chapter II, Proposition 4.9] using Corollary 8.4 and Lemma 10.15 in (i) $\Leftrightarrow$ (ii), Corollary 10.12 and $\omega_{G}=\omega_{G^{\prime}}$ in (ii) $\Leftrightarrow$ (iii), and Lemma 4.10 in (iv) $\Rightarrow$ (iii).

Acknowledgements We are grateful to Alain Genestier and Vincent Lafforgue for many valuable discussions on the topics of the present article. We also thank the referees for allowing us to include formulations from their reports to improve our introduction. 


\section{References}

[EGA] A. Grothendieck: Élements de Géométrie Algébrique, Publ. Math. IHES 4, 8, 11, 17, 20, 24, 28, 32, Bures-Sur-Yvette, 1960-1967; see also Grundlehren 166, Springer-Verlag, Berlin etc. 1971; also available at http://www.numdam.org/numdam-bin/recherche?au=Grothendieck.

[SGA 3] M. Demazure and A. Grothendieck: SGA 3: Schémas en Groupes I, II, III, Lecture Notes in Mathematics, 152, 153. 151, 152, 153, Springer-Verlag, Berlin. 197.

[SGA 6] P. Berthelot, A. Grothendieck, and L. Illusie, eds. SGA 6: Théorie des intersections et théorème de Riemann-Roch, Lecture Notes in Mathematics 225, Springer-Verlag, Berlin, 1971.

[SGA 7] P. Deligne, A. Grothendieck, et al.: SGA 7: Groupes de monodromie en géométrie algébrique, Lecture Notes in Mathematics, 288. , Springer, Berlin-Heidelberg,, 1972;

[Abr06] V. Abrashkin: Galois modules arising from Faltings's strict modules. Compos. Math. 142(2006), no. 4, 867-888; http://dx.doi.org/10.1112/S0010437X06002041

[And86] G. Anderson, $t$-Motives. Duke Math. J. 53 (1986), 457-502. http://dx.doi.org/10.1215/S0012-7094-86-05328-7

[And93] $\longrightarrow$ On Tate modules of formal $t$-modules. Internat. Math. Res. Notices 2(1993), 41-52.

[AH14] E. Arasteh Rad and U. Hartl, Local $\mathbb{P}$-shtukas and their relation to global $\mathfrak{G}$-shtukas. Münster J. Math 7 (2014), 623-670.

[BH09] M. Bornhofen and U. Hartl, Pure Anderson motives over finite fields. J. Number Theory 129, no. 2 (2009), 247-283; also available as arXiv:math.NT/0709.2815. http://dx.doi.org/10.1016/j.jnt.2008.09.006

[Bou61] N. Bourbaki, Éléments de mathématique: Algèbre commutative. Hermann, Paris, 1961.

[CS86] G. Cornell and J. Silverman, eds., Arithmetic geometry. Springer-Verlag, New York, 1986. http://dx.doi.org/10.1007/978-1-4613-8655-1

[CSS97] G. Cornell, J. Silverman, and G. Stevens, eds. Modular forms and Fermat's last theorem. Springer-Verlag, New York, 1997. http://dx.doi.org/10.1007/978-1-4612-1974-3

[Dri74] V. G. Drinfeld, Elliptic modules. Mat. Sb. (N.S.) 23 1974), 594-627.

[Dri77] : - A proof of Langlands' global conjecture for GL(2) over a function field. Funct. Anal. Appl. 11 (1977), no. 3, 223-225. http://dx.doi.org/10.1007/BF01135545

[Dri87] _ Moduli variety of F-sheaves. Funct. Anal. Appl. 21(1987), no. 2, 107-122.

[Eis95] D. Eisenbud, Commutative algebra. Graduate Texts in Mathematics, 150. Springer-Verlag, New York, 1995. http://dx.doi.org/10.1007/978-1-4612-5350-1

[Fal83] G. Faltings, Endlichkeitssätze für abelsche Varietäten über Zahlkörpern. Invent. Math. 73(1983), 349-366. http://dx.doi.org/10.1007/BF01388432

[Fal02] — Group schemes with strict $($-action. Mosc. Math. J. 2(2002), no. 2, 249-279.

[Gen96] A. Genestier, Espaces symétriques de Drinfeld, Astérisque, 234, Soc. Math. France, Paris 1996.

[GL11] A. Genestier and V. Lafforgue, Théorie de Fontaine en égales charactéristiques. Ann. Sci. Éc. Norm. Supér. 44(2011), no. 2, 263-360. http://dx.doi.org/10.24033/asens.2144

[HT01] M. Harris and R. Taylor, The geometry and cohomology of some simple Shimura varieties. Annals of Mathematics Studies, 151. Princeton University Press, Princeton, NJ, 2001.

[Har05] U. Hartl, Uniformizing the stacks of abelian sheaves. In: Number fields and function fields two parallel worlds. Progr. Math., 239, Birkhäuser Boston, Boston, MA, 2005, pp. 167-222;

[Har09] A dictionary between Fontaine-theory and its analogue in equal characteristic. J. Number Theory 129(2009), 1734-1757. http://dx.doi.org/10.1016/j.jnt.2009.01.002

[Har11] Period spaces for Hodge structures in equal characteristic. Annals of Math. 173(2011), n. 3, 1241-1358. http://dx.doi.org/10.4007/annals.2011.173.3.2

[Har17] - Isogenies of abelian Anderson A-modules and A-motives. arxiv:1706.06807 .

[HH16] U. Hartl and S. Hüsken, A criterion for good reduction of Drinfeld modules and Anderson motives in terms of local shtukas. Ann. Sc. Norm. Super. Pisa Cl. Sci. (5) 15(2016), 25-43.

[HJ19] U. Hartl and A.-K. Juschka, Pink's theory of Hodge structures and the Hodge conjecture over function fields. Proceedings of the conference on " $t$-motives: Hodge structures, transcendence and other motivic aspects”, BIRS, Banff, Canada 2009, eds. G. Böckle, D. Goss, U. Hartl, M. Papanikolas, EMS 2019; also available as arxiv:math/1607.01412.

[HK19] U. Hartl and W. Kim. Local Shtukas, Hodge-Pink Structures and Galois representations. Proceedings of the conference on " $t$-motives: Hodge structures, transcendence and other motivic aspects", BIRS, Banff, Canada 2009, eds. G. Böckle, D. Goss, U. Hartl, M. Papanikolas, EMS 2019; also available as arXiv:1512.05893.

[HS] U. Hartl and R. K. Singh, Local shtukas and divisible local Anderson modules. Long version of the present article: arxiv:1511.03697 
[HV11] U. Hartl and E. Viehmann, The Newton stratification on deformations of local G-shtukas. J. Reine Angew. Math. 656(2011), 87-129. http://dx.doi.org/10.1515/crelle.2011.044

[Har77] R. Hartshorne, Algebraic geometry. Graduate Texts in Mathematics, 52. Springer-Verlag, Heidelberg, 1977.

[Ill71] L. Illusie, Complexe cotangent et déformations. I. Lecture Notes in Mathematics, 239. Springer-Verlag, Berlin, 1971.

[Ill72] Complexe cotangent et déformations. II. Lecture Notes in Mathematics, 283. Springer-Verlag, Berlin, 1972.

[Kim09] W. Kim, Galois deformation theory for norm fields and its arithmetic applications. Ph.D. thesis, University of Michigan, Ann Arbor, MI, 2009.

[Laf02] L. Lafforgue, Chtoucas de Drinfeld et correspondance de Langlands. Invent. Math. 147(2002), 1-241. http://dx.doi.org/10.1007/s002220100174

[Laf18] V. Lafforgue, Chtoucas pour les groupes réductifs et paramétrisation de Langlands globale. J. Amer. Math. Soc.31(2018), no. 3, 719-891. http://dx.doi.org/10.1090/jams/897

[Lau96] G. Laumon: Cohomology of Drinfeld modular varieties. I. Cambridge Studies in Advanced Mathematics, 41. Cambridge University Press, Cambridge, 1996.

[LRS93] G. Laumon, M. Rapoport, and U. Stuhler, $\mathcal{D}$-elliptic sheaves and the Langlands correspondence. Invent. Math. 113(1993), 217-338; http://dx.doi.org/10.1007/BF01244308

[LS67] S. Lichtenbaum and M. Schlessinger, The cotangent complex of a morphism. Trans. Amer. Math. Soc. 128(1967) 41-70;. http://dx.doi.org/10.2307/1994516

[Mes72] W. Messing, The crystals associated to Barsotti-Tate groups. Lecture Notes in Mathematics, 264. Springer-Verlag, Berlin, 1972.

[Pog17] T. Poguntke, Group schemes with $\mathbb{F}_{q}$-action. Bull. Soc. Math. France 145(2017), no. 2, 345-380. http://dx.doi.org/10.24033/bsmf.2740

[Qui73] D. Quillen. Higher algebraic K-theory. I. In: Algebraic K-theory, I: higher K-theories. Lecture Notes in Mathematics, 341. Springer, Berlin 1973, pp. 85-147.

[Ros03] M. Rosen, Formal Drinfeld modules. J. Number Theory 103(2003), 234-256. http://dx.doi.org/10.1016/S0022-314X(03)00111-2

[Rot09] J. Rotman, An introduction to homological algebra. Second edition. Springer, New York, 2009. http://dx.doi.org/10.1007/b98977

[Tag93] Y. Taguchi, Semi-simplicity of the Galois representations attached to Drinfeld modules over fields of "infinite characteristics". J. Number Theory 44(1993), no. 3, 292-314. http://dx.doi.org/10.1006/jnth.1993.1055

[Tag95] _ A duality for finite t-modules, J. Math. Sci. Univ. Tokyo 2(1995), 563-588.

[TW96] Y. Taguchi, D. Wan: L-functions of $\varphi$-sheaves and Drinfeld modules, J. Amer. Math. Soc. 9 (1996), no. 3, 755-781; also available at http://www2.math.kyushu-u.ac.jp/ taguchi/bib/. http://dx.doi.org/10.1090/S0894-0347-96-00199-3

[Tat66] J. Tate, p-divisible groups. In: Proc. Conf. Local Fields. Springer, Berlin, 1967, pp. 158-183.

[TW95] R. Taylor and A. Wiles, Ring-theoretic properties of certain Hecke algebras. Ann. of Math. (2) 141(1995), no. 3, 553-572. http://dx.doi.org/10.2307/2118560

[Wil95] A. Wiles, Modular elliptic curves and Fermat's last theorem. Ann. of Math. (2) 141(1995), no. 3, 443-551. http://dx.doi.org/10.2307/2118559

Mathematisches Institut, Universität Münster, Einsteinstr. 62, 48149 Münster, Germany

URL: https://www.uni-muenster.de/Arithm/hartl

Ramakrishna Vivekananda University, PO Belur Math, Dist Howrah, West Bengal 711202, India

Email: rajneeshkumar.s@gmail.com 